September 19, 2002

hep-ph/0209219

\title{
All-Purpose Numerical Evaluation of One-Loop Multi-Leg Feynman Diagrams"
}

\author{
ANDrea FerRogliai \\ Department of Physics, New York University, U.S.A. \\ Massimo Passera \\ Dipartimento di Fisica G. Galilei, Università di Padova, Italy \\ INFN, Sezione di Padova, Italy \\ Giampiero Passarino and SAndro Uccirati \\ Dipartimento di Fisica Teorica, Università di Torino, Italy \\ INFN, Sezione di Torino, Italy
}

\begin{abstract}
A detailed investigation is presented of a set of algorithms which form the basis for a fast and reliable numerical integration of one-loop multi-leg (up to six) Feynman diagrams, with special attention to the behavior around (possibly) singular points in phase space. No particular restriction is imposed on kinematics, and complex masses (poles) are allowed.
\end{abstract}

PACS Classification: 11.10.-z; 11.15.Bt; 12.38.Bx; 02.90.+p

\footnotetext{
${ }^{*}$ Work supported by the European Union under contract HPRN-CT-2000-00149 and by MIUR under contract 2001023713006.

${ }^{\dagger}$ From October 1 2002: Fakultät für Physik, Albert-Ludwigs Universität, Freiburg, Germany.
} 


\section{Contents}

\begin{tabular}{|lr}
\hline Introduction & 2
\end{tabular}

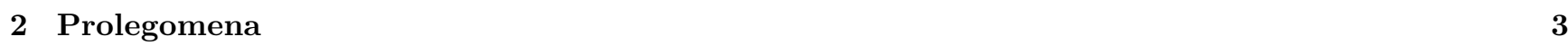

\begin{tabular}{|lrl}
\hline 3 & Generalized two-point function & 5
\end{tabular}

\begin{tabular}{|lrr}
\hline & Three-point functions (C-family) & 8 \\
\hline
\end{tabular}

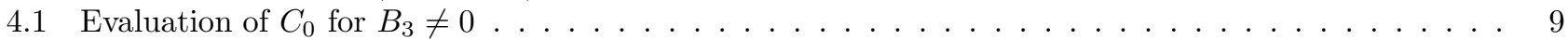

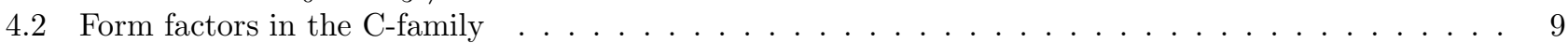

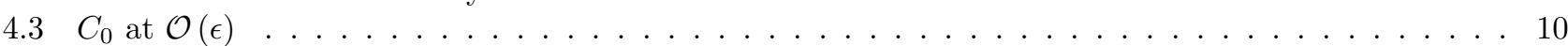

4.4 Evaluation of $C_{0}$ when $B_{3} \approx 0 \quad \ldots \ldots \ldots \ldots \ldots \ldots \ldots \ldots \ldots \ldots \ldots$

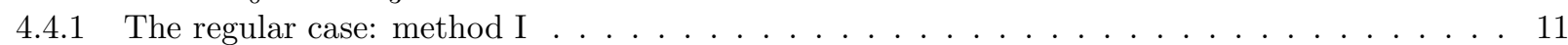

4.4 .2 The regular case: method I . . . . . . . . . . . . . . . . . . . . . . . 11

4.4 .3 The singular case $\ldots \ldots \ldots \ldots \ldots \ldots \ldots \ldots \ldots \ldots \ldots$

$4.5 \quad$ A new integral representation for $C_{0} \ldots \ldots \ldots \ldots \ldots \ldots \ldots \ldots \ldots \ldots$

\begin{tabular}{|l|l}
5 Infrared divergent $C_{0}$ & 21
\end{tabular}

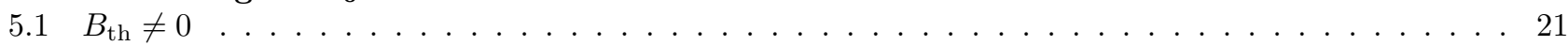

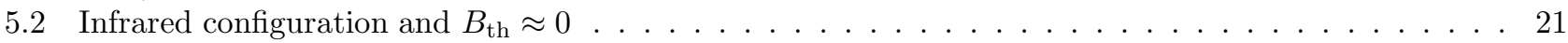

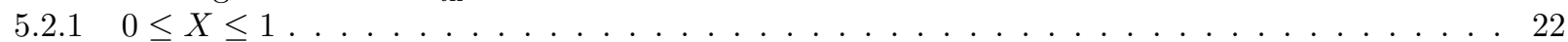

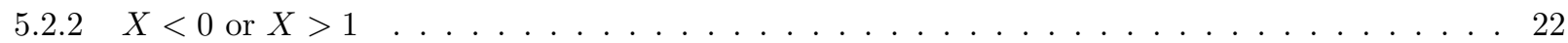

$5.3 \quad$ IR configurations and Landau equations $\ldots \ldots \ldots \ldots \ldots \ldots \ldots \ldots \ldots \ldots$

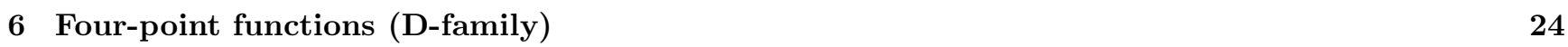

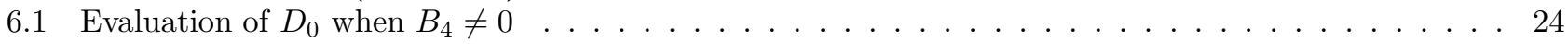

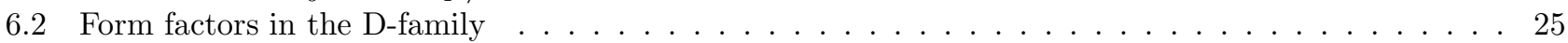

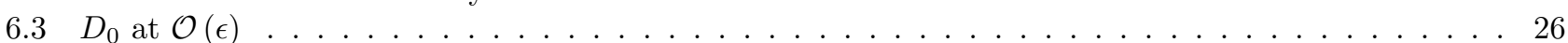

$6.4 \quad$ Evaluation of $D_{0}$ when $B_{4} \approx 0$ and $D_{0}$ is regular $\ldots \ldots \ldots \ldots \ldots \ldots \ldots \ldots \ldots$

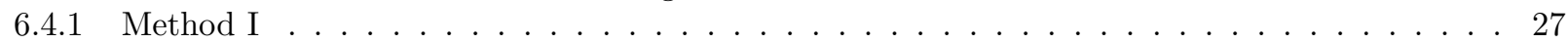

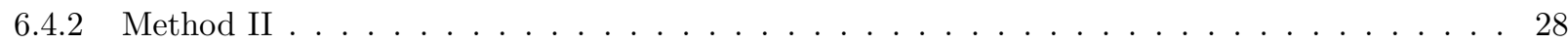

6.4 .3 Sub-leading behavion $\ldots \ldots \ldots \ldots \ldots \ldots$

6.5 Evaluation of $D_{0}$ when $B_{4} \approx 0$ and $D_{0}$ is singular $\ldots \ldots \ldots \ldots \ldots \ldots \ldots \ldots \ldots \ldots \ldots$

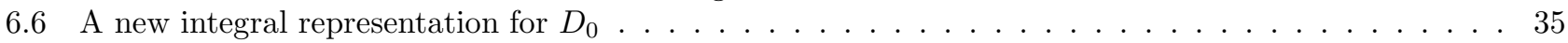

\begin{tabular}{lll}
\hline Infrared divergent $D_{0}$ & 38
\end{tabular}

7.1 Classification of IR divergent cases for the D-family . . . . . . . . . . . . . . . . . . . . . 40

8 Five-point functions (E-family) 41

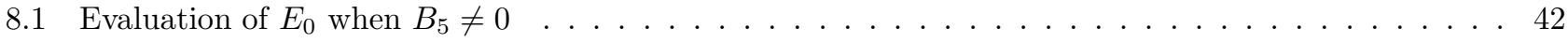

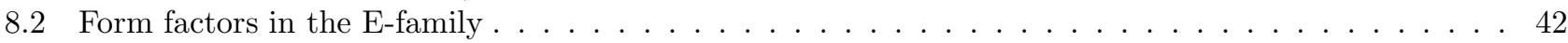

8.3 Evaluation of $E_{0}$ when $B_{5} \approx 0$ and $E_{0}$ is regulan $\ldots \ldots \ldots \ldots \ldots \ldots \ldots \ldots \ldots$

8.4 Evaluation of $E_{0}$ when $B_{5} \approx 0$ and $E_{0}$ is singulan $\ldots \ldots \ldots \ldots \ldots \ldots \ldots \ldots$

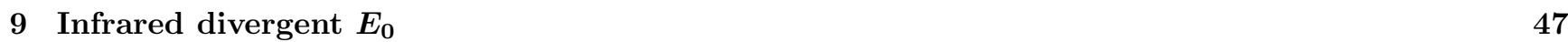

\begin{tabular}{|lr}
\hline 0 Six-point functions (F-family) & 48
\end{tabular}

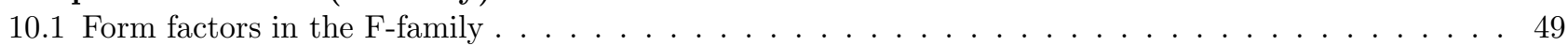

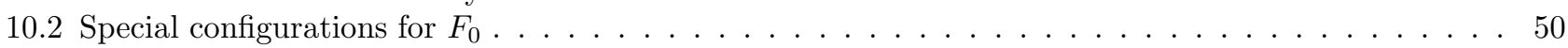

\begin{tabular}{ll}
\hline 11 Conclusions & 51
\end{tabular}

\begin{tabular}{|lr}
\hline A A master integral & 53
\end{tabular} 


\section{Introduction}

In this work we present a systematic approach to the numerical evaluation of one-loop multi-leg Feynman diagrams. One may wonder why or what is new and in what respect there is something to be gained after the seminal work of 't Hooft and Veltman [1], where the analytic answer to the same problem was given.

There are several reasons for attempting a systematization of the problem. First of all we want a fast and reliable way of computing one-loop multi-leg Feynman diagrams for all kinematical configurations, in particular around the (possibly) singular points of the diagrams which are determined by the solution of the corresponding Landau equations [3]. For each graph we have a leading Landau singularity, usually called anomalous threshold for the triangle and higher functions, which for almost all practical situations lies outside the physical region. However, there are important examples where the external legs of the one-loop diagrams are not representing the in/out states of the process under examination and, for a general treatment, we must go beyond the class of $1 \rightarrow 2(3)$ or $2 \rightarrow 2$ processes, to a situation where classifying a priori the physical nature of the anomalous threshold is a tremendous task.

Furthermore we have sub-leading singularities which are the leading ones for the reduced diagrams originating from the primary one 《4. In principle, all of them are fully accounted for by the analytic answer but, in practice, the corresponding numerical evaluation of the special functions that form the answer can be rather unstable. For complicated processes, like full one-loop corrections to $2 \rightarrow 4(6)$ scatterings, we have a rich phase space at our disposal and any automatized calculation must be designed in such a way to foresee all possible sources of numerical problems without having to stop the full evaluation process.

A particularly severe example is represented by the decomposition of pentagons into a sum of boxes [5]. For a real multi-scale problem these boxes are to be evaluated in regions where the analytic result requires cumbersome analytic continuations [6]. Our goal is to derive a numerical answer without having to worry about kinematics. Even more important, our approach does not suffer from the introduction of complex masses which, instead, is a burdensome aspect in the analytic way. At most some care is needed when we take into account that complex masses are to be understood as complex poles lying on the second Riemann sheet [0].

Another well known drawback of the standard analytic approach [8] is the appearance of negative powers of Gram's determinants in the reduction of tensor integrals, which represents a particular annoyance since their zeros do not correspond, under normal circumstances, to true singularities of Feynman diagrams. Clearly, in any approach and after a certain number of symbolic manipulations, certain denominators will show up in the answer. It seems therefore reasonable to design an approach where the zeros of these denominators correspond to true singularities of the diagrams. In any case, a new strategy for the so called problem of reduction of tensor integrals is highly desirable.

Furthermore, in any realistic calculation infrared divergent (IR) virtual corrections will emerge, and we have found it very attractive to use the same tools that are needed to solve the problem of numerical evaluation of multi-scale diagrams for classifying and computing residues of infrared poles and IR finite parts of IR divergent diagrams. In this respect, the interplay between the special class of IR singularities and the general one of Landau singularities is essential.

In this work we make use of a recent proposal for numerical evaluation of multi-loop integrals [9] and extend the work of [10] to all one-loop diagrams (up to six external legs). The global strategy is based on the use of the Bernstein-Tkachov theorem 11] which, away from the leading and sub-leading Landau singularities, allows us to cast any one-loop integrand in a form well suited for numerical treatment. As

\footnotetext{
${ }^{1}$ Alternative approaches, based on differential equation methods, have been originated in [2].
} 
it will be shown in Section 2, a unique factor $B_{G}$ is associated with each one-loop diagram $G$.2 For each family of one-loop diagrams we then consider three different cases: $B_{G} \neq 0, B_{G}=0$ but $G$ regular, and $B_{G}=0$ with $G$ singular. For the last case, the method is based on a particular combination of Mellin-Barnes [12] and sector decomposition [13] techniques, as a result of which we are able to write a Laurent expansion for $G$ around the singularity. Alternatively, we have been able to derive new integral representations that are particularly suited for numerical treatment around the singularities.

To a large extent, the use of complex masses (poles) represents a significant simplification of the whole approach. For physical (real) values of the Mandelstam invariants describing the process, none of the $B_{G}$ factors can be zero, and a straightforward iteration of the BT procedure is all what we need.

To return to our original question on why devoting such an effort to the evaluation of one-loop diagrams, we may add that the results of our work must be seen as one of the many building blocks towards the computation of physical observables at the two-loop level. Here our strategy has been already outlined in [9]: full numerical analysis of the two-loop content. Clearly we will have to include the oneloop part, and it is rather obvious that the two pieces should be treated on equal footing. Furthermore, our main emphasis has been on a general treatment, therefore extending previous works on the same subject [14].

We do not present numbers in this paper for a very simple reason: in all cases where the analytic answer is known, we have found perfect agreement with the one-loop library of TOPAZ0 [15]. The resulting comparison, basically a long list of identical numbers, is not very illuminating.

The outline of the paper will be as follows: in Section 2 we introduce the definitions and discuss the Bernstein-Tkachov theorem, the Mellin-Barnes techniques, and provide a short discussion about the physical/unphysical role of the Landau singularities. In Section 3 the generalized two-point function is introduced, while in Section 4 we start the presentation of the one-loop, three-point, $C$-family, and in Section 5 we discuss its infrared divergent configurations. The four-point, $D$-family, is discussed in Section 6 and the corresponding infrared configurations are presented in Section 7 . The five- and six-point functions are analyzed in Sections 8, 9 and 10.

\section{Prolegomena}

- The Bernstein-Tkachov theorem

The Bernstein-Tkachov theorem [11] (hereafter BT) tells us that for any finite set of polynomials $V_{i}(x)$, where $x=\left(x_{1}, \ldots, x_{k}\right)$ is a vector of Feynman parameters, there exists an identity of the following form:

$$
\mathcal{P}(x, \partial) \prod_{i} V_{i}^{\mu_{i}+1}(x)=B \prod_{i} V_{i}^{\mu_{i}}(x) .
$$

where $\mathcal{P}$ is a polynomial of $x$ and $\partial_{j}=\partial / \partial x_{j} ; B$ and all coefficients of $\mathcal{P}$ are polynomials of $\mu_{i}$ and of the coefficients of $V_{i}(x)$. Any multi-loop Feynman diagram can be cast into the form of Eq.(臬). Iterative applications of the BT functional relations, followed by integration by parts, allows us to express the

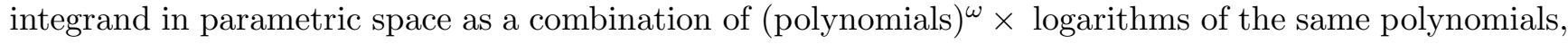
with any given integer $\omega \geq 0$, therefore achieving a result that is well suited for numerical integration. The $B$ coefficients of Eq.(值) are connected to the leading Landau singularity of the corresponding diagram while repeated applications of Eq.(11), after integration by parts, will bring the sub-leading ones into the final answer.

\footnotetext{
${ }^{2}$ This factor is proportional to the determinant of the coefficients in the system of the corresponding Landau equations; S. Uccirati, PhD dissertation (2002).
} 
For general one-loop diagrams we have an explicit solution for the polynomial $\mathcal{P}$ which is due to F. V. Tkachov [11]. Any one-loop Feynman diagram $G$, irrespective of the number $N$ of vertices, can be expressed as

$$
G=\int_{S} d x V^{-\mu}(x)
$$

where the integration region is $x_{j} \geq 0, \sum_{j} x_{j} \leq 1$, with $j=1, \ldots, N-1$, and $V(x)$ is a quadratic polynomial in $x$,

$$
V(x)=x^{t} H x+2 K^{t} x+L .
$$

The solution to the problem of determining the polynomial $\mathcal{P}$ is as follows:

$$
\mathcal{P}=1-\frac{(x-X)^{t} \partial_{x}}{2(\mu+1)}, \quad X^{t}=-K^{t} H^{-1}, \quad B=L-K^{t} H^{-1} K,
$$

where the matrix $H$ is symmetric. In our conventions a multi-leg function is specified by the number of its internal lines, without regard to the actual number of external lines (see Fig. 2 for an illustration).

- Mellin-Barnes transform

Another tool that will be needed in the following is represented by the Mellin-Barnes [12] splitting [16],

$$
(Q+\lambda)^{-\alpha}=\frac{1}{2 \pi i} \int_{-i \infty}^{+i \infty} d s B(s, \alpha-s) \rho^{\alpha-s} Q^{-s}, \quad \alpha>0
$$

where $\rho=1 / \lambda, B$ is the Euler beta-function and

$$
0<\operatorname{Re} s<\alpha, \quad|\arg Q-\arg \lambda|<\pi .
$$

The $i \delta$ prescription is essential in deriving the correct analytic continuation. In all cases $Q$ will be a quadric in $n$ variables and $\lambda$ will be a constant, essentially a BT factor. Therefore $Q$ is always understood as $Q-i \delta$ with $\delta \rightarrow 0_{+}$. Since $|\arg Q-\arg \lambda|<\pi$ is required we will assign a small negative imaginary parts to both $Q$ and $\lambda$.

- Anomalous threshold

For a given one-loop diagram $G$, let us denote with $B_{G}$ the corresponding BT factor of Eq.(1) which is a function of the internal masses and external momenta of $G$. At the leading Landau singularity of $G$, the so-called anomalous threshold (AT) [17], we have $B_{G}=0$. Conversely, $B_{G}=0$ is the condition to have a proper solution for the system of Landau equations corresponding to $G$ (see Section 5.3 for additional discussions). Note that AT is not directly related through unitarity to physical processes (cut diagrams). Most of our work is devoted to analyze specific algorithms for the numerical evaluation of $G$ around $B_{G}=0$ for arbitrary values of the internal masses and external momenta. However, for a large class of applications these parameters are bounded to the physical region $\mathcal{R}$ and the relevant question is whether or not AT $\in \mathcal{R}$. A complete discussion of this issue is technically rather complicated and beyond the scope of our work; here we illustrate a simple case, namely the one-loop vertex of Fig. 2 with the following configuration, $m_{i}=m, p_{2,3}^{2}=-M^{2} \leq 0$ and $p_{1}^{2}=-r$. For real vectors $p_{2,3}$ it follows that $\mathcal{R}$ is defined by either $r \geq 4 M^{2}$ (s-channel) or $r \leq 0$ ( $t$-channel). The diagram has an AT at

$$
r=r_{A T}=4 M^{2}\left(1-\frac{M^{2}}{4 m^{2}}\right), \quad \text { iff } \quad M^{2}>2 m^{2} .
$$

\footnotetext{
${ }^{3}$ In our metric spacelike $p$ implies positive $p^{2}$. Further $p_{4}=i p_{0}$ with $p_{0}$ real for a physical four-momentum.
} 
The kind of singularity depends on the value of $M^{2}$ : if $0<M^{2}<2 m^{2}$ there is no AT, if $2 m^{2}<M^{2}<4 m^{2}$ there is an unphysical AT $\left(0<r_{A T}<4 m^{2}\right)$ and, finally, if $M^{2}>4 m^{2}$ there is a physical AT at $r_{A T}<0$.

- Notations

Finally, to keep our results as compact as possible, we introduce the following notations:

$$
\int d S_{n} \equiv \int_{0}^{1} d x_{1} \int_{0}^{x_{1}} d x_{2} \cdots \int_{0}^{x_{n-1}} d x_{n}, \quad \int d C_{n} \equiv \int_{0}^{1} \prod_{i=1}^{n} d x_{i}, \quad \int d \bar{S}_{2} \equiv \int_{0}^{1} d x_{2} \int_{0}^{x_{2}} d x_{1} .
$$

Likewise we also need

$$
\int d S_{n}(X) \equiv \int_{-X_{1}}^{1-X_{1}} d x_{1} \int_{-X_{2}}^{x_{1}+X_{1}-X_{2}} d x_{2} \cdots \int_{-X_{n}}^{x_{n-1}+X_{n-1}-X_{n}} d x_{n}
$$

where $X$ is an $n$-dimensional vector.

\section{Generalized two-point function}

One of the building blocks for the evaluation of all one-loop diagrams is the generalized two-point function, i.e. a self-energy graph with arbitrary powers for the two internal propagators. The generalized

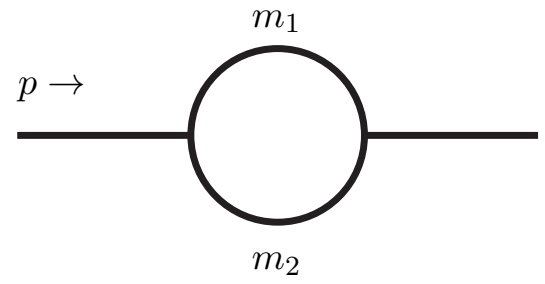

Figure 1: The two-point Green function.

two-point function can be written in terms of the functions

$$
B_{n}(\alpha)=\int_{0}^{1} d x x^{n}\left[\chi_{B}(x)-i \delta\right]^{-\alpha}
$$

where $n$ is a non-negative integer and $\chi_{B}(x)$ is a quadratic polynomial whose coefficients depend on $p^{2}$, the external squared four-momentum, and $m_{1,2}$, the internal masses (see Fig. 1). Let $x_{ \pm}$be the roots of the equation $\chi_{B}-i \delta=0$. If $\lambda\left(-p^{2}, m_{1}^{2}, m_{2}^{2}\right)=0$, where $\lambda(x, y, z)=x^{2}+y^{2}+z^{2}-2(x y+x z+y z)$ is the usual Källen function, the integration contour may be pinched between the two singularities $x_{ \pm}$as $\delta \rightarrow 0_{+}$. If this pinching occurs, the integral is singular with a branch point of the two-particle cut [18]. Consider

$$
\chi_{B}(x)=a x^{2}+b x+c=a(x-X)^{2}+\lambda,
$$

where we have introduced the auxiliary quantities

$$
X=-\frac{b}{2 a}, \quad \lambda=-\frac{\Delta}{4 a}, \quad \Delta=b^{2}-4 a c=\lambda\left(-p^{2}, m_{1}^{2}, m_{2}^{2}\right) .
$$

In the following we describe a solution for $B_{n}(\alpha)$ which allows to extract its behavior around $\lambda=0$. 
We start our derivation by analyzing $B_{0}(\alpha)$. First we assume $0 \leq X \leq 1$ and obtain

$$
B_{0}(\alpha)=\sum_{l=1}^{2}(-1)^{l} X_{l} \int_{0}^{1} d x\left(a X_{l}^{2} x^{2}+\lambda-i \delta\right)^{-\alpha}=\sum_{l=1}^{2}(-1)^{l} X_{l} J_{l}(\alpha, \lambda),
$$

where $X_{1}=-X, X_{2}=1-X$ and where the $i \delta$ prescription is equivalent to the replacement $a, \lambda \rightarrow$ $a, \lambda-i \delta$. In the following it must be understood that both $a$ and $\lambda$ are given an infinitesimal imaginary part. Note that with this prescription we have

$$
\left(\frac{\lambda}{a}\right)^{1 / 2} \equiv\left[\theta\left(\frac{\lambda}{a}\right)-i \operatorname{sign}(a) \theta\left(-\frac{\lambda}{a}\right)\right]\left|\frac{\lambda}{a}\right|^{1 / 2}=\lambda^{1 / 2} a^{-1 / 2} .
$$

Next we split $J_{l}$ into two pieces

$$
J_{l}(\alpha, \lambda)=\int_{0}^{\infty} d x\left(a X_{l}^{2} x^{2}+\lambda\right)^{-\alpha}-\int_{1}^{\infty} d x\left(a X_{l}^{2} x^{2}+\lambda\right)^{-\alpha}
$$

where the first one contains the divergent part for $\lambda \rightarrow 0$, while the second is finite. In the latter we perform the transformation $x \rightarrow x^{-1 / 2}$ and we obtain:

$$
J_{l}(\alpha, \lambda)=\int_{0}^{\infty} d x\left(a X_{l}^{2} x^{2}+\lambda\right)^{-\alpha}-\frac{1}{2} \int_{0}^{1} d x x^{\alpha-3 / 2}\left(a X_{l}^{2}+\lambda x\right)^{-\alpha} .
$$

The first integral in Eq.(15) can be cast into the following form,

$$
\int_{0}^{\infty} d x\left(a X_{l}^{2} x^{2}+\lambda\right)^{-\alpha}=\frac{1}{2} \frac{\lambda^{1 / 2-\alpha}}{a^{1 / 2}\left|X_{l}\right|} B\left(\frac{1}{2}, \alpha-\frac{1}{2}\right),
$$

which is valid for $|\arg (\lambda / a)|<\pi$ and $\operatorname{Re} \alpha>1 / 2$. Here $B$ denotes the Euler beta-function. Collecting the two pieces we obtain

$$
\begin{aligned}
B_{0}(\alpha) & =\frac{1}{2} \sum_{l=1}^{2} \frac{(-1)^{l} X_{l}}{\left|X_{l}\right|}\left[\lambda^{1 / 2-\alpha} a^{-1 / 2} B\left(\frac{1}{2}, \alpha-\frac{1}{2}\right)-\left|X_{l}\right| \int_{0}^{1} d x x^{\alpha-3 / 2}\left(a X_{l}^{2}+\lambda x\right)^{-\alpha}\right] \\
& =\lambda^{1 / 2-\alpha} a^{-1 / 2} B\left(\frac{1}{2}, \alpha-\frac{1}{2}\right)-\frac{1}{2} \sum_{l=1}^{2}\left|X_{l}\right| \int_{0}^{1} d x x^{\alpha-3 / 2}\left(a X_{l}^{2}+\lambda x\right)^{-\alpha},
\end{aligned}
$$

where we have used the relation $\left|X_{l}\right|=(-1)^{l} X_{l}$, consequence of $0 \leq X \leq 1$. If instead $X<0$ or $X>1$ the first term vanishes and there is no divergence. In general we can write

$$
\int_{0}^{1} d x x^{\alpha-3 / 2}\left(a X_{l}^{2}+\lambda x\right)^{-\alpha}=\frac{1}{\alpha-1 / 2}\left(a X_{l}^{2}\right)^{-\alpha}{ }_{2} F_{1}\left(\alpha, \alpha-\frac{1}{2} ; \alpha+\frac{1}{2} ;-\frac{\lambda}{a X_{l}^{2}}\right),
$$

where ${ }_{2} F_{1}$ is the standard hypergeometric function [12]. Let us define $z_{l}=-\lambda /\left(a X_{l}^{2}\right)$; then, for $\alpha=1$ we obtain

$$
{ }_{2} F_{1}\left(1, \frac{1}{2} ; \frac{3}{2} ; z_{l}\right)=\frac{1}{2} z_{l}^{-1 / 2} \ln \frac{1+z_{l}^{1 / 2}}{1-z_{l}^{1 / 2}},
$$

while for $\alpha=n$ we will use

$$
{ }_{2} F_{1}\left(n, n-\frac{1}{2} ; n+\frac{1}{2} ; z_{l}\right)=\frac{(3 / 2)_{n-1}}{(1)_{n-1}(1 / 2)_{n-1}} \frac{d^{n-1}}{d z_{l}^{n-1}}{ }_{2} F_{1}\left(1, \frac{1}{2} ; \frac{3}{2} ; z_{l}\right),
$$


where $(a)_{n}=\Gamma(a+n) / \Gamma(a)$ is the Pochhammer symbol and $\Gamma$ is the Euler gamma-function. Note that both integrals diverge for $\alpha \rightarrow 1 / 2$ and that the divergent parts cancel in the sum. At $\alpha=1 / 2$ and with $\xi_{l}^{2}=a X_{l}^{2} / \lambda$ we have the following result:

$$
B_{0}\left(\frac{1}{2}\right)=\sum_{l=1}^{2}(-1)^{l} \operatorname{sign}\left(X_{l}\right) a^{-1 / 2} \ln \left[\xi_{l}+\left(1+\xi_{l}^{2}\right)^{1 / 2}\right] .
$$

In the same way we find that for the general case the result is

$$
\begin{aligned}
B_{n}(\alpha)= & \int_{0}^{1} d x x^{n}\left[\chi_{B}(x)-i \delta\right]^{-\alpha}=\frac{1}{2} \sum_{l=1}^{2} \sum_{k=0}^{n}\left(\begin{array}{l}
n \\
k
\end{array}\right)(-1)^{l} X^{n-k}\left[\frac{X_{l}}{\left|X_{l}\right|}\right]^{k+1} \\
& {\left[\lambda^{k / 2+1 / 2-\alpha} a^{-k / 2-1 / 2} B\left(\frac{k+1}{2}, \alpha-\frac{k+1}{2}\right)-\left|X_{l}\right|^{k+1} \int_{0}^{1} d x x^{\alpha-\frac{3+k}{2}}\left(a X_{l}^{2}+\lambda x\right)^{-\alpha}\right] . }
\end{aligned}
$$

The integral in the second term of Eq.(23) can also be expressed through an hypergeometric function:

$$
\begin{aligned}
B_{n}(\alpha)= & \int_{0}^{1} d x x^{n}\left[\chi_{B}(x)-i \delta\right]^{-\alpha}=\frac{1}{2} \sum_{l=1}^{2} \sum_{k=0}^{n}\left(\begin{array}{l}
n \\
k
\end{array}\right)(-1)^{l} X^{n-k}\left[\frac{X_{l}}{\left|X_{l}\right|}\right]^{k+1} \\
& {\left[\lambda^{k / 2+1 / 2-\alpha} a^{-k / 2-1 / 2} B\left(\frac{k+1}{2}, \alpha-\frac{k+1}{2}\right)-\right.} \\
& \left.\left|X_{l}\right|^{k+1} \frac{\left(a X_{l}\right)^{-\alpha}}{\alpha-(k+1) / 2}{ }_{2} F_{1}\left(\alpha, \alpha-\frac{k+1}{2} ; \alpha-\frac{k-1}{2} ;-\frac{\lambda}{a X_{l}^{2}}\right)\right] .
\end{aligned}
$$

Finally, a special case will be needed in dealing with multi-leg functions: let $\alpha=1+\epsilon / 2$ and consider the expansion around $\epsilon=0$; for $n=0$, we obtain

$$
\begin{gathered}
B_{0}(1+\epsilon / 2)=B_{0}(1)+\frac{\epsilon}{2} B_{0}^{\prime}(1)+\mathcal{O}\left(\epsilon^{2}\right), \\
B_{0}^{\prime}(1)=\pi\left[\psi\left(\frac{1}{2}\right)-\psi(1)-\ln (\lambda-i \delta)\right](\lambda a)^{-1 / 2}-\frac{1}{2} \sum_{i=1}^{2}\left|X_{i}\right| \\
\times \sum_{n=0}^{\infty} \frac{\left(a X_{i}^{2}\right)^{-n-1}}{n+1 / 2}\left[\psi(n+1)-\psi(1)-\frac{1}{n+1 / 2}-\ln \left(a X_{i}^{2}-i \delta\right)\right](-\lambda)^{n},
\end{gathered}
$$

where $\psi$ is the Euler psi-function. More generally, for a non-negative integer value of $n$, we have

$$
\begin{gathered}
B_{n}(1+\epsilon / 2)=B_{n}(1)+\frac{\epsilon}{2} B_{n}^{\prime}(1)+\mathcal{O}\left(\epsilon^{2}\right), \\
B_{n}^{\prime}(1)=\frac{1}{2} \sum_{l=1}^{2} \sum_{k=0}^{n}\left(\begin{array}{l}
n \\
k
\end{array}\right)(-1)^{l} X^{n-k}\left[\frac{X_{l}}{\left|X_{l}\right|}\right]^{k+1}\left(b_{1 ; k}^{\prime}+b_{2 ; k}^{\prime}\right),
\end{gathered}
$$

where $b_{1 ; k}^{\prime}$ and $b_{2 ; k}^{\prime}$ are the terms proportional to $\epsilon / 2$ that are obtained by expanding around $\epsilon=0$ the first and second term in the square brackets in Eq.(24), respectively:

$$
b_{1 ; k}^{\prime}=\lambda^{k / 2-1 / 2} a^{-k / 2-1 / 2} \mathrm{~B}\left(\frac{1-k}{2}, \frac{1+k}{2}\right)\left[\psi\left(\frac{1-k}{2}\right)-\psi(1)-\ln (\lambda-i \delta)\right],
$$




$$
\begin{aligned}
b_{2 ; k}^{\prime} & =\left|X_{l}\right|^{k+1} \sum_{m=0}^{\infty} \frac{2}{a X_{l}^{2}(k-1-2 m)^{2}}\left(-\frac{\lambda}{a X_{l}}\right)^{m}\{2+(k-1-2 m)[\psi(m+1) \\
& \left.\left.-\psi(1)-\ln \left(a X_{l}^{2}-i \delta\right)\right]\right\} .
\end{aligned}
$$

\section{Three-point functions (C-family)}

We introduce scalar one-loop $N$-point functions, with $N \geq 3$, according to the following definition:

$$
\begin{aligned}
S_{N} & =\frac{1}{i \pi^{2} \Gamma(N-2)} \int d^{d} q \frac{1}{\left[q^{2}+m_{1}^{2}\right] \cdots\left[\left(q+p_{1}+\cdots+p_{N-1}\right)^{2}+m_{N}^{2}\right]} \\
& =\int d S_{N-1} V_{N}^{2-N-\epsilon / 2}(x),
\end{aligned}
$$

where $d=4-\epsilon$ is the space-time dimensionality and $V_{N}(x)$ is a quadratic polynomial in the Feynman parameters $x_{1}, \ldots, x_{N-1}$. In this section we analyze an arbitrary scalar three-point function $C_{0} \equiv S_{3}$ (see Fig. 2), defined by

$$
C_{0}=\int d S_{2} V^{-1-\epsilon / 2}\left(x_{1}, x_{2}\right),
$$

where $V$ is a quadratic polynomial

$$
V\left(x_{1}, x_{2}\right)=a x_{1}^{2}+b x_{2}^{2}+c x_{1} x_{2}+d x_{1}+e x_{2}+f-i \delta \equiv x^{t} H x+2 K^{t} x+L,
$$

whose coefficients are related to the internal masses and the external momenta by the relations $H_{i j}=$ $-p_{i} \cdot p_{j}, L=m_{1}^{2}$ and

$$
K_{1}=\frac{1}{2}\left(p_{1} \cdot p_{1}+m_{2}^{2}-m_{1}^{2}\right), \quad K_{2}=\frac{1}{2}\left(P \cdot P-p_{1} \cdot p_{1}+m_{3}^{2}-m_{2}^{2}\right),
$$

with $P=p_{1}+p_{2}$.
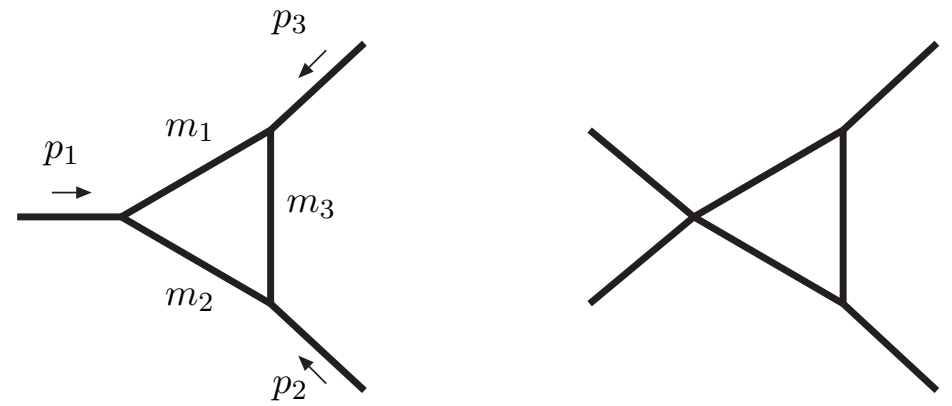

Figure 2: The one-loop, three-point Green function. The second diagram, although having 4 external lines, is included in the $C$-family of 3 internal lines.

Let us define the usual BT factors t as $B_{3}=L-K^{t} H^{-1} K$ and $X=-H^{-1} K$. In the following we will present integral representations for $C_{0}$ which are well suited for numerical integration and cover all options for the choice of external momenta.

\footnotetext{
${ }^{4}$ Note, however, the different sign in the definition of $X$ with respect to 11 .
} 


\subsection{Evaluation of $C_{0}$ for $B_{3} \neq 0$}

If $B_{3} \neq 0$ we can rewrite $C_{0}$ according to the standard BT procedure of Eq.(1). It is convenient to introduce special notations, $X_{0}=1, X_{3}=0$, and $V(\widehat{i i+1})$ to denote contractions, i.e.

$$
V(\widehat{01})=V\left(1, x_{1}\right), \quad V(\widehat{12})=V\left(x_{1}, x_{1}\right), \quad V(\widehat{23})=V\left(x_{1}, 0\right) .
$$

In this way we obtain a simple integral representation

$$
C_{0}=\frac{1}{B_{3}}\left\{\frac{1}{2}+\int_{0}^{1} d x_{1}\left[\int_{0}^{x_{1}} d x_{2} \ln V\left(x_{1}, x_{2}\right)-\frac{1}{2} \sum_{i=0}^{2}\left(X_{i}-X_{i+1}\right) \ln V(\widehat{i i+1})\right]\right\} .
$$

Note that a zero of the corresponding Gram's determinant is not a problem, actually it even decreases the number of terms. Indeed let $G_{3}=\operatorname{det} H$ and denote by $\Delta_{i j}$ the co-determinant of the element $H_{i j}$. If $G_{3}=0$ we obtain

$$
C_{0}\left(G_{3}=0\right)=-\frac{1}{2 b_{3}} \int_{0}^{1} d x \sum_{i=0}^{2}\left(\mathcal{X}_{i}-\mathcal{X}_{i+1}\right) \ln V(\widehat{i i+1})
$$

where $B_{3}=b_{3} / G_{3}$ and $\mathcal{X}=-\Delta K$, showing that, in this case, $C_{0}$ is a combination of three $B_{0}$ functions [19].

\subsection{Form factors in the C-family}

The most general $C$-family integral that we consider in any field theory is

$$
C\left[Q\left(x_{1}, x_{2}\right)\right]=\int d S_{2} Q\left(x_{1}, x_{2}\right) V^{-1-\epsilon / 2}\left(x_{1}, x_{2}\right), \quad Q\left(x_{1}, x_{2}\right)=\sum_{n=1}^{\bar{n}} \sum_{m=1}^{\bar{m}} a_{n m} x_{1}^{n} x_{2}^{m} .
$$

The integral representation corresponding to Eq.(35) will be

$$
C\left[Q\left(x_{1}, x_{2}\right)\right]=\sum_{n=1}^{\bar{n}} \sum_{m=1}^{\bar{m}} \frac{a_{n m}}{B_{3}}\left[C_{m, n}^{0}-\frac{1}{2} \int d S_{1} C_{m, n}^{1}-\frac{1}{2} \int d S_{2} x_{1}^{n-1} x_{2}^{m-1} C_{m, n}^{2}\right],
$$

where the $C$-coefficients are

$$
\begin{aligned}
C_{m, n}^{0} & =\frac{1}{(2+n+m)(1+m)}, \\
C_{m, n}^{1} & =\left(X_{1}-X_{2}\right) x_{1}^{n+m} \ln V\left(x_{1}, x_{1}\right)+\delta_{m, 0} X_{2} x_{1}^{n} \ln V\left(x_{1}, 0\right)+\left(1-X_{1}\right) x_{1}^{m} \ln V\left(1, x_{1}\right), \\
C_{m, n}^{2} & =\left(m X_{2} x_{1}-(2+n+m) x_{1} x_{2}+n X_{1} x_{2}\right) \ln V\left(x_{1}, x_{2}\right) .
\end{aligned}
$$

Examples of standard form factors of the $C$-family (see [8 for the general definition) are

$$
B_{3} C_{i j}=\int d S_{2} c_{i j}^{k} \ln V\left(x_{1}, x_{2}\right)+\int d S_{1} \sum_{l=0}^{2} c_{i j}^{l} \ln V(\widehat{i+1+1})+K_{i j}
$$


with coefficients

$$
\begin{aligned}
c_{11}^{k} & =\frac{1}{2}\left(X_{1}-3 x_{1}\right), \quad c_{11}^{1}=\frac{1}{2} X_{12} x_{1}, \quad c_{11}^{2}=\frac{1}{2} X_{2} x_{1}, \quad c_{11}^{0}=\frac{1}{2} X_{01}, \quad K_{11}=-\frac{1}{3}, \\
c_{12}^{k} & =\frac{1}{2}\left(X_{2}-3 x_{2}\right), \quad c_{12}^{1}=\frac{1}{2} X_{12} x_{1}, \quad c_{12}^{2}=0, \quad c_{12}^{0}=\frac{1}{2} X_{01} x_{1}, \quad K_{12}=-\frac{1}{6}, \\
c_{21}^{k} & =x_{1}\left(2 x_{1}-X_{1}\right), \quad c_{21}^{1}=-\frac{1}{2} X_{12} x_{1}^{2}, \quad c_{21}^{2}=-\frac{1}{2} X_{2} x_{1}^{2}, \quad c_{21}^{0}=-\frac{1}{2} X_{01}, \quad K_{21}=\frac{1}{4}, \\
c_{22}^{k} & =x_{2}\left(2 x_{2}-X_{2}\right), \quad c_{22}^{1}=-\frac{1}{2} x_{1}^{2} X_{12}, \quad c_{22}^{2}=0, \quad c_{22}^{0}=-\frac{1}{2} X_{01} x_{1}^{2}, \quad K_{22}=\frac{1}{12}, \\
c_{23}^{k} & =2 x_{1} x_{2}-\frac{1}{2}\left(X_{1} x_{2}+X_{2} x_{1}\right), \\
c_{23}^{1} & =-\frac{1}{2} x_{1}^{2} X_{12}, \quad c_{23}^{2}=0, \quad c_{23}^{0}=-\frac{1}{2} X_{01} x_{1}, \quad K_{23}=\frac{1}{8},
\end{aligned}
$$

where we introduced $X_{i j}=X_{i}-X_{j}$ and $X_{0}=1$. For $C_{24}$ we directly obtain

$$
C_{24}=\frac{1}{4} \frac{1}{\bar{\varepsilon}}-\frac{1}{2} \int d S_{2} \ln V\left(x_{1}, x_{2}\right), \quad \frac{1}{\bar{\varepsilon}}=\frac{2}{\varepsilon}-\gamma-\ln \pi,
$$

where $\gamma=0.577216 \cdots$ is the Euler constant and $d=4-\epsilon$ is the space-time dimensionality.

It is perhaps the right moment to summarize our strategy for tensor integrals. Clearly a decomposition in form factors is always possible and, within our approach, the form factors are not plagued by the appearance of inverse powers of Gram's determinants. However, this is not exactly the way a calculation should be organized since one has to profit from the strategy of the BT procedure by mapping all integrals, for all topologies, into just one integral. Therefore, there is no need to perform a pre-reduction in momentum space. Furthermore, in any realistic calculation, the denominators in Feynman diagrams contain scalar products, e.g. $q \cdot p_{1}$ etc, and one has to benefit as much as possible from algebraic simplification. However, this is not always the case because each of the external lines, say in our original diagram, can split into other two (three) lines (we have in mind vector bosons that should be considered as unstable particles and, therefore, always be attached to fermionic currents). In this case momenta will appear that are not present in the propagators of the diagram and no simplification will occur. For this reason, and for the reader's convenience, we list all the form factors in the one-loop families.

\section{$4.3 \quad C_{0}$ at $\mathcal{O}(\epsilon)$}

A two-point function with the insertion of a counterterm, which is needed in a two-loop calculation, is formally identical to a one-loop three-point function with one zero external momentum. Its expression at $\mathcal{O}(\epsilon)$ is needed and this follows from the BT algorithm quite nicely,

$$
\begin{gathered}
C_{0}(d)=C_{0}(4)+\epsilon C_{0}^{(1)}+\mathcal{O}\left(\epsilon^{2}\right) \\
C_{0}^{(1)}=-\frac{1}{2} \int d S_{2}\left[\ln V\left(x_{1}, x_{2}\right)+\frac{1}{2} \ln ^{2} V\left(x_{1}, x_{2}\right)\right] \\
+\frac{1}{8} \int d S_{1} \sum_{i=0}^{2}\left(X_{i}-X_{i+1}\right) \ln ^{2} V(\widehat{i i+1})
\end{gathered}
$$

showing that it contains at most squares of logarithms of quadratic forms. 


\subsection{Evaluation of $C_{0}$ when $B_{3} \approx 0$}

When we are around the region where $B_{3}=0$, Eq.(35) cannot be used and an alternative derivation is needed. Actually there are two sub-cases to be discussed starting from the observation that $C_{0}$ can be written as

$$
C_{0}=\int d S_{2}\left[(x-X)^{t} H(x-X)+B_{3}\right]^{-1-\epsilon / 2} .
$$

If $B_{3}=0$ the integral of Eq.(45) is singular or regular depending on the values of $X_{1,2}$.

\subsubsection{The regular case: method I}

If $B_{3}=0$, but the condition

$$
0 \leq X_{2} \leq X_{1} \leq 1
$$

is not fulfilled, there is no real singularity inside the integration domain $\mathcal{I}_{3}$; still we cannot apply the standard BT techniques of Eq.(1). The integral in Eq.(31) is well defined through distortion of the contour integration and there is no pinch inside $\mathcal{I}_{3}$. We use this regularity to write the following BT relations, corresponding to $B_{3} \neq 0$ and $B_{3}=0$ :

$$
\begin{aligned}
& {\left[1-\frac{1}{2(\mu+1)}(x-X) \partial_{x}\right] V^{\mu+1}\left(x_{1}, x_{2}\right)=B_{3} V^{\mu}\left(x_{1}, x_{2}\right),} \\
& {\left[1-\frac{1}{2(\mu+1)}(x-X) \partial_{x}\right] V_{0}^{\mu+1}\left(x_{1}, x_{2}\right)=0}
\end{aligned}
$$

with $V=V_{0}+B_{3}$. Subtracting the two equations, we obtain:

$$
V^{\mu}\left(x_{1}, x_{2}\right)=\left[1-\frac{1}{2(\mu+1)}(x-X) \partial_{x}\right] \frac{V^{\mu+1}\left(x_{1}, x_{2}\right)-V_{0}^{\mu+1}\left(x_{1}, x_{2}\right)}{B_{3}} .
$$

Note that this relation holds even in the case $B_{3} \sim 0$. Since the point of coordinates $x_{i}=X_{i}$ is outside $\mathcal{I}_{3}$, we can perform an integration by parts on both $V$ and $V_{0}$. The result is the following:

$$
C_{0}=\frac{1}{B_{3}}\left[\int d S_{2} \ln \frac{V}{V_{0}}-\frac{1}{2} \int d S_{1} \sum_{i=0}^{2}\left(X_{i}-X_{i+1}\right) \ln \frac{V(\widehat{i i+1})}{V_{0}(\widehat{i+1+1})}\right] .
$$

All tensor integrals can be treated according to the same procedure. Note that the logarithms appearing in Eq.(49) are of the form $\ln (1+x)$ with small $|x|$ and the corresponding values must retain full relative precision, e.g. they must be computed with routines based on Chebyshev expansion.

\subsubsection{The regular case: method II}

Even if the method of Section 4.4.1 gives a compact result for the regular case, we derive another expression for numerical checking of our results. Since $C_{0}$ is not singular in the limit $B_{3} \rightarrow 0$ we can use a Taylor expansion of the integrand of Eq.(31) followed by a BT-algorithm corresponding to $B_{3}=0$. Therefore we have:

$$
C_{0}=\sum_{n=0}^{\infty}\left(-B_{3}\right)^{n} \int d S_{2}(X)\left(x^{t} H x\right)^{-n-1-\epsilon / 2}=\sum_{n=0}^{\infty} \mathcal{C}(n+1)\left(-B_{3}\right)^{n}
$$


where we kept $\epsilon \neq 0$ only where strictly needed and where we used Eq.(9). By using:

$$
\int d S_{2}(X)\left[1+\frac{1}{2 n+\epsilon} x \partial_{x}\right] V^{-n-\epsilon / 2}\left(x_{1}, x_{2}\right)=0 .
$$

We observe that integration-by-parts is allowed since $x_{i}=0$ is outside the integration domain and derive, after changing $x$ into either $x-X_{1}$ or $x-X_{2}$,

$$
\mathcal{C}(n)=\int d S_{2}(X) V^{-n-\epsilon / 2}\left(x_{1}, x_{2}\right)=-\frac{1}{2 n-2+\epsilon} \int d S_{1} \sum_{i=0}^{2}\left(X_{i}-X_{i+1}\right) V^{-n-\epsilon / 2}(T \widehat{i i+1}),
$$

where we employed $X_{0}=1$ and $X_{3}=0$, and we introduced shifted arguments, i.e. the three relevant quadratic forms in $x$ are

$$
\begin{aligned}
& V(T \widehat{01}) \equiv V_{c}=V\left(1-X_{1}, x-X_{2}\right), \quad V(T \widehat{12}) \equiv V_{a}=V\left(x-X_{1}, x-X_{2}\right), \\
& V(T \widehat{23}) \equiv V_{b}=V\left(x-X_{1},-X_{2}\right),
\end{aligned}
$$

for which we write $V_{i}=h_{i} x^{2}+2 k_{i} x+l_{i}$ and introduce the standard BT factor, $b_{i}, i=a, b, c$, and also the BT co-factor, $X_{i}, i=a, b, c$. As long as all the $b_{i}$ are different from zero, the corresponding BT relations hold. For the first term in the expansion we obtain:

$$
\begin{gathered}
\mathcal{C}(1)=\frac{1}{2} \sum_{i=a, b, c} \frac{X_{i}^{L}}{b_{i}}\left[\mathcal{C}_{0 ; i}(1)+\int_{0}^{1} d x \mathcal{C}_{1 ; i}(1, x)\right], \\
\mathcal{C}_{0 ; i}(1)=-\frac{1}{4}\left[X_{i} \ln ^{2} V_{i}(0)+\left(1-X_{i}\right) \ln ^{2} V_{i}(1)\right], \quad \mathcal{C}_{1 ; i}(1, x)=\ln V_{i}(x)+\frac{1}{4} \ln ^{2} V_{i}(x),
\end{gathered}
$$

where the leading coefficients are:

$$
X_{a}^{L}=X_{1}-X_{2}, \quad X_{b}^{L}=X_{2}, \quad X_{c}^{L}=1-X_{1},
$$

whereas for $n>1$ we get

$$
\begin{gathered}
\mathcal{C}(n)=-\frac{1}{4(n-1)^{2}} \sum_{i=a, b, c} \frac{X_{i}^{L}}{b_{i}}\left[\mathcal{C}_{0 ; i}(n)+\int_{0}^{1} d x \mathcal{C}_{1 ; i}(n, x)\right], \\
\mathcal{C}_{0 ; i}(n)=\left(1-X_{i}\right) V_{i}^{-n+1}(1)+X_{i} V_{i}^{-n+1}(0), \quad \mathcal{C}_{1 ; i}(1, x)=(2 n-3) V_{i}^{-n+1}(x) .
\end{gathered}
$$

Now we can sum the series according to the well-known results:

$$
\sum_{n=1}^{\infty} \frac{x^{n}}{n}=-\ln (1-x), \quad \sum_{n=1}^{\infty} \frac{x^{n}}{n^{2}}=\operatorname{Li}_{2}(x),
$$

where $\operatorname{Li}_{2}(x)$ is the standard dilogarithm [20], and obtain a novel representation for $C_{0}$ :

$$
\begin{gathered}
C_{0}=\frac{1}{2} \sum_{i=a, b, c} \frac{X_{i}^{L}}{b_{i}}\left[C_{0 ; i}+\int_{0}^{1} d x C_{1 ; i}\right], \\
C_{0 ; i}=-\frac{1}{4} X_{i}\left[2 \operatorname{Li}_{2}\left(-\frac{B_{3}}{V_{i}(0)}\right)+\ln ^{2} V_{i}(0)\right]-\frac{1}{4}\left(1-X_{i}\right)\left[2 \operatorname{Li}_{2}\left(-\frac{B_{3}}{V_{i}(1)}\right)+\ln ^{2} V_{i}(1)\right], \\
C_{1 ; i}=\ln \left(V_{i}(x)+B_{3}\right)+\frac{1}{2} \operatorname{Li}_{2}\left(-\frac{B_{3}}{V_{i}(x)}\right)+\frac{1}{4} \ln ^{2} V_{i}(x) .
\end{gathered}
$$


The above derivation of the coefficients in the Taylor expansion, Eq.(52), assumes that all sub-leading BT factors are non zero. $\mathcal{C}(n)$ of Eq. (52) is a combination of generalized two-point functions of Section 3; if one of the $b_{i} \approx 0$ we still have to distinguish between two possibilities:

a) $b_{i}=0$ but $-h_{i} \leq k_{i} \leq 0\left(h_{i} \geq 0\right)$ is not fulfilled.

b) $b_{i}=0$ and $-h_{i} \leq k_{i} \leq 0\left(h_{i} \geq 0\right)$ is fulfilled.

In both cases we can apply the results of Section 3. So let us denote each term of Eq.(52) with

$$
\mathcal{C}^{i}(n)=\frac{1}{2 n-2+\epsilon} \int_{0}^{1} d x g_{i} V_{i}^{-n-\epsilon / 2}(x),
$$

where $g_{i}=-X_{i}+X_{i+1}$. The corresponding contribution to $C_{0}$ will be

$$
C_{0}^{i}=\int_{0}^{1} d x g_{i} V_{i}^{-1-\epsilon / 2}(x) \sum_{n=0}^{\infty} \frac{\left(-B_{3}\right)^{n}}{2 n+\epsilon} V_{i}^{-n}(x) .
$$

In evaluating the sum it is more convenient to isolate the leading term $C_{0}^{i}(n=0)$ and consider the rest separately. The latter becomes

$$
\begin{aligned}
{\left[C_{0}^{i}\right]_{\mathrm{rest}}=} & \frac{1}{2} g_{i} \sum_{n=1}^{\infty} \frac{\left(-B_{3}\right)^{n}}{n} \int_{0}^{1} d x V_{i}^{-n-1}(x)=\frac{1}{2} g_{i} \sum_{l=1}^{2} \frac{(-1)^{l} X_{i}^{l}}{\left|X_{i}^{l}\right|} \sum_{n=1}^{\infty} \frac{\left(-B_{3}\right)^{n}}{n} \\
& {\left[\frac{1}{2} b_{i}^{-1 / 2-n} h_{i}^{-1 / 2} B\left(\frac{1}{2}, n+\frac{1}{2}\right)-\left|X_{i}^{l}\right| \int_{0}^{1} d x x^{2 n}\left(h_{i} X_{i}^{l^{2}}+b_{i} x^{2}\right)^{-n-1}\right], }
\end{aligned}
$$

where $X_{1 i}=-X_{i}$ and $X_{2 i}=1-X_{i}$. The second series can be easily summed up, while for the first one we have:

$$
\begin{aligned}
\sum_{n=1}^{\infty} \frac{\left(-B_{3} / b_{i}\right)^{n}}{n} B\left(\frac{1}{2}, n+\frac{1}{2}\right) & =\pi^{1 / 2} \sum_{n=0}^{\infty} \frac{(n+1 / 2) \Gamma(n+1 / 2)}{(n+1)^{2} \Gamma(n+1)}\left(-\kappa_{i}\right)^{n+1} \\
& =-\pi \kappa_{i} \int_{0}^{1} d x\left(1+\frac{1}{2} \ln x\right)\left(1+\kappa_{i} x\right)^{-1 / 2}
\end{aligned}
$$

where we introduced $\kappa_{i}=B_{3} / b_{i}$. Finally we obtain:

$$
\begin{aligned}
{\left[C_{0}^{i}\right]_{\mathrm{rest}}=} & -\frac{1}{2} g_{i} \sum_{l=1}^{2} \frac{(-1)^{l} X_{l i}}{\left|X_{l i}\right|} \int_{0}^{1} d x\left[\frac{\pi \kappa_{i}}{\left(b_{i} h_{i}\right)^{1 / 2}} \frac{x+x \ln x}{\left(1+\kappa_{i} x^{2}\right)^{1 / 2}}\right. \\
& \left.-\left|X_{l i}\right|\left(h_{i} X_{l i}^{2}+b_{i} x^{2}\right)^{-1} \ln \frac{h_{i} X_{l i}^{2}+\left(b_{i}+B_{3}\right) x^{2}}{h_{i} X_{l i}^{2}+b_{i} x^{2}}\right] .
\end{aligned}
$$

As we argued for the generalized two-point function, the divergent term for $b_{i} \approx 0$ cancels out if the corresponding $X_{i}$ is outside the interval $[0,1]$. For the leading term, after an $\epsilon$ expansion, we have:

$$
C_{0}^{i}(n=0)=\int_{0}^{1} d x g_{i} V_{i}^{-1}(x)\left[\frac{1}{\epsilon}-\frac{1}{2} \ln V_{i}\right], \quad \sum_{i=0}^{2} \int_{0}^{1} d x g_{i} V_{i}^{-1}=0 .
$$


Note that the divergent term does not contribute to $C_{0}$. In order to calculate the term $C_{0}^{i}(n=0)$, we need the relevant part of $\mathcal{C}(1)$, i.e. the one corresponding to those values of $i$ for which $b_{i} \approx 0$. The latter contribution can be obtained by observing that, symbolically,

$$
\epsilon \mathcal{C}(1)=\sum B_{0}(1+\epsilon / 2)
$$

using Eq. 26) we expand the relevant generalized two-point function and derive an expression for $\mathcal{C}(1)$ by equating the terms proportional to $\epsilon$.

Also for the form factors we can derive a similar expansion. Consider the auxiliary function

$$
\begin{aligned}
C\left(n_{1}, n_{2}\right) & =\sum_{n=0}^{\infty}\left(-B_{3}\right)^{n} \int d S_{2}(X) \prod_{i=1}^{2}\left(x_{i}+X_{i}\right)^{n_{i}}\left(x^{t} H x\right)^{-n-1-\epsilon / 2} \\
& =\sum_{n=0}^{\infty} \sum_{l_{1}=0}^{n_{1}} \sum_{l_{2}=0}^{n_{2}}\left(\begin{array}{c}
n_{1} \\
l_{1}
\end{array}\right)\left(\begin{array}{c}
n_{2} \\
l_{2}
\end{array}\right) X_{1}^{n_{1}-l_{1}} X_{2}^{n_{2}-l_{1}} \mathcal{C}\left(n+1, l_{1}, l_{2}\right)\left(-B_{3}\right)^{n},
\end{aligned}
$$

and the expansion is fully specified by

$$
\begin{aligned}
\mathcal{C}\left(n, n_{1}, n_{2}\right) & =\frac{1}{2-2 n+n_{1}+n_{2}} \int_{0}^{1} d x\left\{\left[\left(x-X_{1}\right)^{n_{1}}\left(x-X_{2}\right)^{n_{2}}\left(X_{1}-X_{2}\right) V_{a}^{-n}\right.\right. \\
& \left.-\left(x-X_{1}\right)^{n_{1}}\left(-X_{2}\right)^{n_{2}+1} V_{b}^{-n}+\left(x-X_{2}\right)^{n_{2}}\left(1-X_{1}\right)^{n_{1}+1} V_{c}^{-n}\right\} .
\end{aligned}
$$

Combinations of these functions account for all form factors.

\subsubsection{The singular case}

If condition Eq.(46) is fulfilled then there is a pinch inside $\mathcal{I}_{3}$ and the integral in Eq.(31) is singular. In order to minimize the number of terms in the solution we write

$$
\begin{aligned}
C_{0} & =\int d S_{2} V^{-1-\epsilon / 2}\left(x_{1}, x_{2}\right)=\int d C_{2} V^{-1-\epsilon / 2}\left(x_{1}, x_{2}\right)-\int_{0}^{1} d x_{1} \int_{x_{1}}^{1} d x_{2} V^{-1-\epsilon / 2}\left(x_{1}, x_{2}\right) \\
& =\int d C_{2} V^{-1-\epsilon / 2}\left(x_{1}, x_{2}\right)-\int d S_{2} V^{-1-\epsilon / 2}\left(x_{2}, x_{1}\right)=C_{0}^{\text {square }}-C_{0}^{\text {comp }} .
\end{aligned}
$$

Since the point $x_{i}=X_{i}$ is now internal to the integration domain the complementary $C_{0}$ function will be regular and can be computed according to the results of Section 4.4.1 and 4.4.2. A special case is represented by $0 \leq X_{2}=X_{1} \leq 1$ where both the original $C_{0}$ and the complementary one are singular. However, due to the symmetry of $H$, we easily obtain

$$
C_{0}=C_{0}^{\text {comp }}=\frac{1}{2} C_{0}^{\text {square }}, \quad \text { for } \quad 0 \leq X_{2}=X_{1} \leq 1 .
$$

$C_{0}^{\text {square }}$ is the integral over $[0,1]^{2}$ which we rewrite by changing variables, $x_{i}^{\prime}=x_{i}-X_{i}$, as

$$
C_{0}^{\text {square }}=\sum_{i=1}^{4} \alpha_{i} \beta_{i} \int d C_{2}\left[Q_{i}\left(x_{1}, x_{2}\right)+B_{3}\right]^{-1-\epsilon / 2},
$$

where

$$
\alpha_{1}=\alpha_{2}=1-X_{1}, \quad \alpha_{3}=\alpha_{4}=X_{1}, \quad \beta_{1}=\beta_{3}=1-X_{2}, \quad \beta_{2}=\beta_{4}=X_{2},
$$


and where the new quadrics are defined by

$$
\begin{aligned}
& Q_{1}=Q\left(\left(1-X_{1}\right) x_{1},\left(1-X_{2}\right) x_{2}\right), \quad Q_{2}=Q\left(\left(1-X_{1}\right) x_{1},-X_{2} x_{2}\right), \\
& Q_{3}=Q\left(-X_{1} x_{1},\left(1-X_{2}\right) x_{2}\right), \quad Q_{4}=Q\left(-X_{1} x_{1},-X_{2} x_{2}\right),
\end{aligned}
$$

with $Q=x^{t} H x$. In general we define

$$
Q_{i}\left(x_{1}, x_{2}\right)=A_{i} x_{1}^{2}+B_{i} x_{2}^{2}+C_{i} x_{1} x_{2} .
$$

In order the derive a Laurent expansion around $B_{3}=0$ we introduce $\rho_{3}=1 / B_{3}$ and perform a MellinBarnes splitting as described in Section 2,

$$
C_{0}^{\text {square }}=\sum_{i=1}^{4} \frac{\alpha_{i} \beta_{i}}{2 \pi i} \int_{-i \infty}^{+i \infty} d s B(s, 1-s) \rho_{3}^{1-s} \mathcal{C}_{i}(s), \quad \mathcal{C}_{i}(s)=\int d C_{2} Q_{i}^{-s}\left(x_{1}, x_{2}\right) .
$$

Let us consider in detail the $\mathcal{C}_{i}$-functions. We use a simple sector decomposition [13] to obtain

$$
\begin{aligned}
\mathcal{C}_{i} & =\left[\int d S_{2}+\int d \bar{S}_{2}\right] Q_{i}^{-s}\left(x_{1}, x_{2}\right)=\int d C_{2} x_{1}^{1-2 s}\left(A_{i}+C_{i} x_{2}+B_{i} x_{2}^{2}\right)^{-s} \\
& +\int d C_{2} x_{2}^{1-2 s}\left(B_{i}+C_{i} x_{1}+A_{i} x_{1}^{2}\right)^{-s}=\frac{1}{2(1-s)} \sum_{j=1}^{2} \mathcal{C}_{i, j}(s)
\end{aligned}
$$

For each of the $\mathcal{C}_{i j}$-functions we have a reduced quadratic form in one variable. Let us postpone for a moment the problem of their evaluation. From Eq. (76]) we obtain

$$
\begin{aligned}
C_{0}^{\text {square }} & =\frac{1}{2} \sum_{i=1}^{4} \sum_{j=1}^{2} \alpha_{i} \beta_{i} \frac{1}{2 \pi i} \int_{-i \infty}^{+i \infty} d s \frac{\Gamma(s) \Gamma(1-s)}{1-s} \rho_{3}^{1-s} \mathcal{C}_{i j}(s), \\
\mathcal{C}_{i j}(s) & =\int_{0}^{1} d x\left(h_{i j} x^{2}+2 k_{i j} x+l_{i j}\right)^{-s}
\end{aligned}
$$

and $0<\operatorname{Re} s<1$. Suppose that the corresponding BT factors,

$$
b_{i j}=l_{i j}-k_{i j}^{2} / h_{i j}=\alpha_{i}^{2} \beta_{i}^{2} \operatorname{det}(H) / h_{i j}
$$

are not zero and that we are interested in the region of large $\left|\rho_{3}\right|$. Then we close the integration contour over the right-hand complex half-plane at infinity. The poles are at $s=1$ (double) and at $s=k+1$ (single) where $k \geq 1$ is an integer. For $\mathcal{C}_{i j}$ we use

$$
\begin{aligned}
\mathcal{C}_{i j}(s) & =\frac{1}{b_{i j}} \int_{0}^{1} d x\left[1+\frac{1}{2} \frac{x-X_{i j}}{s-1} \frac{d}{d x}\right] Q_{i j}^{1-s}(x), \\
Q_{i j}(x) & =h_{i j} x^{2}+2 k_{i j} x+l_{i j},
\end{aligned}
$$

where $X_{i j}$ is the BT co-factor for $Q_{i j}$. In the limit $s \rightarrow 1$ we obtain

$$
\begin{aligned}
& \mathcal{C}_{i j}(s)=\mathcal{C}_{i j}(1)+(s-1) \mathcal{C}_{i j}^{\prime}(1)+\mathcal{O}\left((s-1)^{2}\right), \\
& \mathcal{C}_{i j}(1)=\frac{1}{b_{i j}} \int_{0}^{1} d x\left[1-\frac{1}{2}\left(x-X_{i j}\right) \frac{d}{d x} \ln Q_{i j}(x)\right], \\
& \mathcal{C}_{i j}^{\prime}(1)=\frac{1}{b_{i j}} \int_{0}^{1} d x\left[-\ln Q_{i j}(x)+\frac{1}{4}\left(x-X_{i j}\right) \frac{d}{d x} \ln ^{2} Q_{i j}(x)\right] .
\end{aligned}
$$


Therefore the residue of the double pole at $s=1$ is

$$
\left.\mathcal{R}_{i j}\right|_{s=1}=\mathcal{C}_{i j}(1) \ln \rho_{3}-\mathcal{C}^{\prime}{ }_{i j}(1) .
$$

For the single poles at $s=k+1, k \geq 1$ we find that the residues are

$$
\left.\mathcal{R}_{i j}\right|_{s=k+1}=-\frac{(-1)^{k}}{k} B_{0}(k+1) \rho_{3}^{-k},
$$

where $B_{0}(k+1)$ is a generalized two-point function discussed in Section 3. With the help of Eqs.(78)-(83) we obtain the full Laurent expansion.

Also for the form factors we can use the same strategy and write the auxiliary functions $C\left(n_{1}, n_{2}\right)$ as a combinations of Mellin-Barnes integrals

$$
\begin{array}{r}
\int_{-i \infty}^{+i \infty} d s \frac{\Gamma(s) \Gamma(1-s)}{1-s+\left(n_{1}+n_{2}\right) / 2} \rho_{3}^{1-s} \mathcal{C}_{i j}\left(n_{j}, s\right), \\
\mathcal{C}_{i j}\left(n_{j}, s\right)=\int_{0}^{1} d x x^{n_{j}}\left(h_{i j} x^{2}+2 k_{i j} x+l_{i j}\right)^{-s} .
\end{array}
$$

The relevant poles are at $s=k+1, k \geq 0$ and there are two possibilities: if $n_{1}+n_{2}=2 n$ then there is a double pole for $s=n+1$, otherwise, if $n_{1}+n_{2}=2 n+1$, there is one additional, simple, pole at $s=n+3 / 2$.

However, the secondary BT factors, $b_{i j}$, could be near to zero. Note that the $b_{i j}$ coefficients are sub-leading of a second type since $b_{i j}=0$ should not be confused, in general, with the condition for the occurence of a Landau singularity associated with a reduced diagram. Indeed, following Eq. (73) and Eq.(79), they correspond to having $0=X_{2} \leq X_{1} \leq 1$ or $0 \leq X_{2} \leq X_{1}=1$ in the pinch singularity and it is enough to select $m_{i}=m, \forall i$ in Eq.(33) to see that there is no correspondence with any of the two-particle cuts. From Eq.(㱏) and Eq.(79) it is easily seen that for $G_{3}=\operatorname{det}(H) \sim 0$ we hace $b_{i j} \sim G_{3}^{-3}$ so that $G_{3}=0$ does not imply $b_{i j}=0$.

$b_{i j}=0$ alone is not yet a sign of instability because $\mathcal{C}_{i j}$ will show a pinch singularity in [0,1] only if $h_{i j}>0$ and $-h_{i j} \leq k_{i j} \leq 0$. If we are in this region for some pair $i j$ then it is better to use another expansion algorithm. From Eq.(76) we see that the total result is a sum of terms with the following structure:

$$
\begin{aligned}
\rho F(\rho) & =\frac{1}{2 \pi i} \int_{-i \infty}^{+i \infty} d s \frac{\Gamma(s) \Gamma(1-s)}{s-1} f(s) \rho^{1-s}, \\
f(s) & =\int_{0}^{1} d x\left[h\left(x+\frac{k}{h}\right)^{2}+b\right]^{-s}=\int_{\alpha}^{1+\alpha} d x\left(h x^{2}+b\right)^{-s},
\end{aligned}
$$

where $\alpha=k / h$. With $\rho_{S L}=1 / b$ we write

$$
f(s)=\rho_{S L}^{s}\left\{\int_{0}^{1+\alpha}-\int_{0}^{\alpha}\right\} d x\left(1+\rho_{S L} h x^{2}\right)^{-s} .
$$

Introducing a second Mellin-Barnes splitting gives

$$
\begin{aligned}
\mathcal{F}\left(\rho, \rho_{S L}\right) & \equiv \rho F(\rho)=-\sum_{n=1}^{2}(-1)^{n} \frac{\rho \beta_{n}}{2} \frac{1}{(2 \pi i)^{2}} \int_{-i \infty}^{+i \infty} d s d t \\
& \times \frac{\Gamma(1-s) \Gamma(t) \Gamma(s-t)}{(s-1)(t-1 / 2)}\left(\frac{\rho_{S L}}{\rho}\right)^{s}\left(\rho_{S L} h \beta_{n}^{2}\right)^{-t},
\end{aligned}
$$


with $\beta_{1}=\alpha$ and $\beta_{2}=1+\alpha$. This representation is valid in the vertical strip $0<\operatorname{Re} t<\operatorname{Re} s<1$ and $\operatorname{Re} t<1 / 2$. Since we are interested in the region where both the leading $B$ and the sub-leading $b$ are approaching zero, we close the $t$-contour over the right-hand complex half-plane at infinity with poles at $t=1 / 2$ and $t=s+k, k \geq 0$. We choose $\operatorname{Re} s>1 / 2$ and obtain

$$
\begin{aligned}
\mathcal{F}\left(\rho, \rho_{S L}\right) & \equiv \rho F(\rho)=\sum_{n=1}^{2}(-1)^{n} \frac{\rho \beta_{n}}{2} \frac{1}{2 \pi i} \int_{-i \infty}^{+i \infty} d s \frac{\Gamma(1-s)}{s-1}\left(\frac{\rho_{S L}}{\rho}\right)^{s} \\
& \times\left[\pi^{1 / 2} \Gamma\left(s-\frac{1}{2}\right)\left(h \beta_{n}^{2} \rho_{S L}\right)^{-1 / 2}+\sum_{k=0}^{\infty} \frac{(-1)^{k+1}}{k !} \frac{\Gamma(s+k)}{s+k-1 / 2}\left(h \beta_{n}^{2} \rho_{S L}\right)^{-s-k}\right] .
\end{aligned}
$$

$\mathcal{F}$ is naturally split into two parts:

$$
\begin{aligned}
\mathcal{F}\left(\rho, \rho_{S L}\right) & =\sum_{n=1}^{2}(-1)^{n}\left[\mathcal{F}_{1}^{(n)}+\sum_{k=0}^{\infty} \frac{(-1)^{k}}{k !} \mathcal{F}_{2}^{(n, k)}\right] \\
& =\sum_{n=1}^{2}(-1)^{n} \frac{\rho \beta_{n}}{2}\left[\pi^{1 / 2}\left(h \beta_{n}^{2} \rho_{S L}\right)^{-1 / 2} \mathcal{F}_{1, n}+\sum_{k=0}^{\infty} \frac{(-1)^{k}}{k !} \mathcal{F}_{2, n, k}\right]
\end{aligned}
$$

For the first part in Eq. (89) we introduce the new variable $\kappa=\rho_{S L} / \rho$ and derive the following coefficient:

$$
\mathcal{F}_{1, n}=\frac{1}{2 \pi i} \int_{-i \infty}^{+i \infty} d s \frac{\Gamma(1-s) \Gamma(s-1 / 2)}{s-1} \kappa^{s} .
$$

We close the contour over the left-hand complex half-plane at infinity. with poles at $s=1 / 2-k, k \geq 0$, obtaining

$$
\begin{aligned}
\mathcal{F}_{1, n} & =-\sum_{k=0}^{\infty} \frac{(-1)^{k}}{k !} \frac{\Gamma(k+1 / 2)}{k+1 / 2} \kappa^{1 / 2-k}=-\kappa^{1 / 2} \sum_{k=0}^{\infty} \frac{\Gamma^{2}(k+1 / 2)}{\Gamma(k+3 / 2)} \frac{(-\kappa)^{-k}}{k !} \\
& =-2 \pi \kappa^{1 / 2}{ }_{2} F_{1}\left(\frac{1}{2}, \frac{1}{2} ; \frac{3}{2} ;-\frac{1}{\kappa}\right)=-2 \pi^{1 / 2} \kappa \ln \frac{1+(1+\kappa)^{1 / 2}}{\kappa^{1 / 2}}
\end{aligned}
$$

As a consequence we have

$$
\mathcal{F}_{1}^{(n)}=-\operatorname{sign}\left(\beta_{n}\right) \pi\left(\frac{\rho_{S L}}{h}\right)^{1 / 2} \ln \frac{\rho^{1 / 2}+\left(\rho+\rho_{S L}\right)^{1 / 2}}{\rho_{S L}^{1 / 2}} .
$$

For the second part in Eq.(89) the poles are at $s=l+1, l \geq 0$ where $l=0$ gives a double pole. We obtain

$$
\begin{aligned}
\mathcal{F}_{2, n, k} & =-\frac{\Gamma(k+1)}{k+1 / 2}\left(h \beta_{n}^{2}\right)^{-k-1} \rho^{-1} \rho_{S L}^{-k}\left[-\frac{1}{k+1 / 2}+\gamma+\psi(k+1)-\ln \left(h \beta_{n}^{2} \rho\right)\right] \\
& -\sum_{l=1}^{\infty} \frac{(-1)^{l}}{l !} \frac{\Gamma(k+l+1)}{l(k+l+1 / 2)}\left(h \beta_{n}^{2}\right)^{-k-l-1} \rho^{-l-1} \rho_{S L}^{-k} .
\end{aligned}
$$

Substituting back into Eq. (76) gives the requested result.

There is another situation that requires particular care: consider a quadratic form $h x^{2}+2 k x+l$ and suppose that $b \neq 0$ but that $l \approx 0$ or that, equivalently $h+2 k+l \approx 0$. The solution that amounts to 
iterate the BT procedure for $f(k)$ is unstable because of the surface terms in the integration by parts. In this case we take the Mellin-Barnes transform of the integrand and write

$$
\begin{aligned}
f(s) & =\frac{1}{2 \pi i} \int_{-i \infty}^{+i \infty} d t B(t, s-t) L^{s-t} \int_{0}^{1} d x x^{-t}(2 k+h x)^{-t} \\
& =\frac{1}{2 \pi i} \int_{-i \infty}^{+i \infty} d t B(t, s-t) L^{s-t} \frac{(2 k)^{-t}}{1-t}{ }_{2} F_{1}\left(t, 1-t ; 2-t ;-\frac{h}{2 k}\right),
\end{aligned}
$$

where $L=1 / l$ and $0<\operatorname{Re} t<\operatorname{Re} s<1$. Therefore we obtain

$$
\begin{aligned}
\mathcal{F}(\rho, L) & \equiv \rho F(\rho)=-\frac{\rho}{(2 \pi i)^{2}} \int_{-i \infty}^{+i \infty} d s d t \frac{\Gamma(1-s) \Gamma(t) \Gamma(s-t)}{(s-1)(t-1)} \\
& \times\left(\frac{L}{\rho}\right)^{s}(2 k L)^{-t}{ }_{2} F_{1}\left(t, 1-t ; 2-t ;-\frac{h}{2 k}\right) .
\end{aligned}
$$

For large $|L|$ we close the $t$-integration contour over the right-hand complex half-plane at infinity with poles at $t=1$ and $t=s+n$. We get

$$
\begin{aligned}
\mathcal{F}(\rho, L) & =-\frac{\rho}{(2 \pi i)^{2}} \int_{-i \infty}^{+i \infty} d s \frac{\Gamma(1-s)}{s-1}\left(\frac{L}{\rho}\right)^{s}\left[-\Gamma(s-1)(2 k L)^{-1}+\sum_{n=0}^{\infty} \frac{(-1)^{n}}{n !} \Gamma(s+n-1)\right. \\
& \left.\times(2 k L)^{-s-n}{ }_{2} F_{1}\left(s+n, 1-s-n ; 2-s-n ;-\frac{h}{2 k}\right)\right] .
\end{aligned}
$$

For the first term we write

$$
\frac{1}{2 \pi i} \int_{-i \infty}^{+i \infty} d s \frac{\Gamma(1-s) \Gamma(s-1)}{s-1}\left(\frac{L}{\rho}\right)^{s}=\sum_{n=1}^{\infty} \frac{1}{n^{2}}\left(-\frac{L}{\rho}\right)^{1-n}=-\frac{L}{\rho} \operatorname{Li}_{2}\left(-\frac{\rho}{L}\right),
$$

where we closed the $s$-integration contour over the left-hand complex half-plane at infinity, with poles at $s=1-n, n \geq 1$. For the second term we need special cases of the hypergeometric function [12]:

$$
{ }_{2} F_{1}(-n, 1+n ; 2+n ; z)=\frac{1}{(2+n)_{n}}\left(\frac{1-z}{z}\right)^{n+1} \frac{d^{n}}{d z^{n}}\left[\frac{z^{2 n+1}}{1-z}\right] .
$$

\subsection{A new integral representation for $C_{0}$}

It is very important to derive our results, for all kinematical regions, in more than one form in order to be able to provide reliable cross-checks. For this reason we derived another representation for $C_{0}$, to be used when the BT coefficient $B_{3}$ vanishes and the integral is singular. Starting from Eq.(75) a sector decomposition of the square $[0,1]^{2}$ gives

$$
\begin{aligned}
C_{0}^{\text {square }} & =\sum_{i=1}^{4} \alpha_{i} \beta_{i}\left(\int d S_{2}+\int d \bar{S}_{2}\right)\left[Q_{i}\left(x_{1}, x_{2}\right)+B_{3}\right]^{-1-\epsilon / 2} \\
& =\sum_{i=1}^{4} \sum_{j=1}^{2} \alpha_{i} \beta_{i} \int d C_{2} x_{2}\left[x_{2}^{2} Q_{i j}\left(x_{1}\right)+B_{3}\right]^{-1-\epsilon / 2},
\end{aligned}
$$

where we introduced $Q_{i 1}\left(x_{1}\right)=Q_{i}\left(x_{1}, 1\right)$ and $Q_{i 2}\left(x_{1}\right)=Q_{i}\left(1, x_{1}\right)$. They have the following form: $Q_{i j}\left(x_{1}\right)=h_{i j} x_{1}^{2}+2 k_{i j} x_{1}+l_{i j}$. Note that, according to the $i \delta$ prescription, the following replacement must always be understood: $Q_{i j}, B_{3} \rightarrow Q_{i j}, B_{3}-i \delta$. Now we use the results of Appendix A: the coefficients in the corresponding master integral are:

$$
a=Q_{i j}\left(x_{1}\right), \quad b=B_{3}, \quad \alpha=1+\epsilon / 2, \quad \beta=1 .
$$


Therefore we obtain:

$$
\begin{aligned}
C_{0}^{\text {square }} & =\sum_{i=1}^{4} \sum_{j=1}^{2} \alpha_{i} \beta_{i} \int_{0}^{1} d x_{1}\left\{\frac{1}{\epsilon} B_{3}^{-\epsilon / 2} Q_{i j}^{-1}\left(x_{1}\right)-\int_{0}^{1} d x_{2} x_{2}^{-1+\epsilon} Q_{i j}^{-1-\epsilon / 2}\left(x_{1}, x_{2}\right)\right\}, \\
Q_{i j}\left(x_{1}, x_{2}\right) & =Q_{i j}\left(x_{1}\right)+B_{3} x_{2}^{2} .
\end{aligned}
$$

If the factors $b_{i j}=l_{i j}-k_{i j}^{2} / h_{i j}=\alpha_{i}^{2} \beta_{i}^{2} \operatorname{det}(H) / h_{i j}$, corresponding to the quadratic forms $Q_{i j}\left(x_{1}\right)$, are different from zero we apply the following BT relations:

$$
\begin{gathered}
Q_{i j}^{-1}(x)=\frac{1}{b_{i j}}\left[1-\frac{1}{2}\left(x+\frac{k_{i j}}{h_{i j}}\right) \partial_{x} \ln Q_{i j}(x)\right], \\
Q_{i j}^{\mu-1}\left(x_{1}, x_{2}\right)=\frac{1}{b_{i j}}\left\{1-\frac{1}{2 \mu}\left[\left(x_{1}+\frac{k_{i j}}{h_{i j}}\right) \partial_{x_{1}}+x_{2} \partial_{x_{2}}\right]\right\} Q_{i j}^{\mu}\left(x_{1}, x_{2}\right) .
\end{gathered}
$$

Let us introduce new auxiliary variables: $\zeta_{i j 1}=-k_{i j} / h_{i j}$ and $\zeta_{i j 2}=1+k_{i j} / h_{i j}$ to obtain a compact result:

$$
\begin{gathered}
C_{0}^{\text {square }}=\sum_{i=1}^{4} \sum_{j=1}^{2} \frac{\alpha_{i} \beta_{i}}{8 b_{i j}} C_{i j} \\
C_{i j}=2 \ln B_{3}\left[-\int_{0}^{1} d x_{1} \ln Q_{i j}\left(x_{1}\right)+\zeta_{i j 2} \ln Q_{i j}(1)+\zeta_{i j 1} \ln Q_{i j}(0)-2\right] \\
-4 \int_{0}^{1} d x_{1} \int_{0}^{1} d x_{2}\left(\frac{\ln Q_{i j}\left(x_{1}, x_{2}\right)}{x_{2}}\right)_{+}+\int_{0}^{1} d x_{1}\left[\ln ^{2} Q_{i j}\left(x_{1}\right)+4 \ln Q_{i j}\left(x_{1}, 1\right)\right] \\
+4 \int_{0}^{1} d x_{2}\left[\zeta_{i j 2}\left(\frac{\ln Q_{i j}\left(1, x_{2}\right)}{x_{2}}\right)_{+}+\zeta_{i j 1}\left(\frac{\ln Q_{i j}\left(0, x_{2}\right)}{x_{2}}\right)_{+}\right]-\zeta_{i j 2} \ln ^{2} Q_{i j}(1)-\zeta_{i j 1} \ln ^{2} Q_{i j}(0) .
\end{gathered}
$$

All the $Q_{i j}$ and $B_{3}$ are given a $-i \delta$. The ' + '-distribution is defined, as usual, by its action on a generic test function $f(x)$ :

$$
\int_{0}^{1} d x\left(\frac{f(x)}{x}\right)_{+}=\int_{0}^{1} d x \frac{f(x)-f(0)}{x} .
$$

If instead some $b_{i j}$ approaches zero we must repeat the procedure. This can be achieved if we start from Eq.(101) and change variables according to $x_{1} \rightarrow x_{1}-k_{i j} / h_{i j}$. Then we decompose the $x_{1}$ integration interval and remap each piece into the unit square; the result is

$$
\begin{aligned}
C_{0}^{\text {square }}= & \sum_{i=1}^{4} \sum_{j=1}^{2} \sum_{l=1}^{2} \alpha_{i} \beta_{i} \zeta_{i j l} \int_{0}^{1} d x_{1}\left\{\frac{1}{\epsilon} B_{3}^{-\epsilon / 2}\left[h_{i j} \zeta_{i j l}^{2} x_{1}^{2}+b_{i j}-i \delta\right]^{-1}\right. \\
& \left.-\int_{0}^{1} d x_{2} x_{2}^{-1+\epsilon}\left[h_{i j} \zeta_{i j l}^{2} x_{1}^{2}+B_{3} x_{2}^{2}+b_{i j}\right]^{-1-\epsilon / 2}\right\},
\end{aligned}
$$

where the replacement $b_{i j}, h_{i j} \rightarrow b_{i j}, h_{i j}-i \delta$ is also understood. Now we apply the result of Appendix A to both integrals, deriving

$$
C_{0}^{\text {square }}=\sum_{i=1}^{4} \sum_{j=1}^{2} \sum_{l=1}^{2} \alpha_{i} \beta_{i} \zeta_{i j l}\left\{\frac{\pi}{2 \epsilon}\left(h_{i j} \zeta_{i j l}^{2}\right)^{-1 / 2} B_{3}^{-\epsilon / 2} b_{i j}^{-1 / 2}\right.
$$




$$
\begin{aligned}
& -\frac{1}{2} B\left(\frac{1}{2}, \frac{1}{2}+\frac{\epsilon}{2}\right)\left(h_{i j} \zeta_{i j l}^{2}\right)^{-1 / 2} \int_{0}^{1} d x_{2} x_{2}^{-1+\epsilon}\left[B_{3} x_{2}^{2}+b_{i j}\right]^{-1 / 2-\epsilon / 2} \\
& -\frac{1}{\epsilon} B_{3}^{-\epsilon / 2} \int_{0}^{1} d x_{1}\left[h_{i j} \zeta_{i j l}^{2}+b_{i j} x_{1}^{2}\right]^{-1} \\
& \left.+\int_{0}^{1} d x_{1} \int_{0}^{1} d x_{2} x_{1}^{\epsilon} x_{2}^{-1+\epsilon}\left[h_{i j} \zeta_{i j l}^{2}+\left(B_{3} x_{2}^{2}+b_{i j}\right) x_{1}^{2}\right]^{-1-\epsilon / 2}\right\} .
\end{aligned}
$$

The first three terms in Eq.(108) are divergent for $B_{3} \sim b_{i j} \sim 0$. The second integral depends only on the ratio

$$
\kappa_{i j}=\frac{B_{3}-i \delta}{b_{i j}-i \delta}
$$

so that, when we apply the master formula of Appendix A, the result is

$$
\int_{0}^{1} d x_{2} x_{2}^{-1+\epsilon}\left[B_{3} x_{2}^{2}+b_{i j}\right]^{-1 / 2-\epsilon / 2}=\frac{1}{2} b_{i j}^{-1 / 2} B_{3}^{-\epsilon / 2} B\left(\frac{\epsilon}{2}, \frac{1}{2}\right)-\int_{0}^{1} d x_{2}\left[b_{i j} x_{2}^{2}+B_{3}\right]^{-1 / 2-\epsilon / 2} .
$$

Note that the first term in Eq. 110) cancels the first term of Eq.(108):

$$
\begin{aligned}
C_{0}^{\text {square }}= & \sum_{i=1}^{4} \sum_{j=1}^{2} \sum_{l=1}^{2} \alpha_{i} \beta_{i} \zeta_{i j l}\left\{-\frac{1}{\epsilon} B_{3}^{-\epsilon / 2} \int_{0}^{1} d x_{1}\left[h_{i j} \zeta_{i j l}^{2}+b_{i j} x_{1}^{2}\right]^{-1}\right. \\
& +\frac{1}{2} B\left(\frac{1}{2}, \frac{1}{2}+\frac{\epsilon}{2}\right)\left(h_{i j} \zeta_{i j l}^{2}\right)^{-1 / 2} \int_{0}^{1} d x_{2}\left[b_{i j} x_{2}^{2}+B_{3}\right]^{-1 / 2-\epsilon / 2} \\
& \left.+\int_{0}^{1} d x_{1} \int_{0}^{1} d x_{2} x_{1}^{\epsilon} x_{2}^{-1+\epsilon}\left[h_{i j} \zeta_{i j l}^{2}+\left(B_{3} x_{2}^{2}+b_{i j}\right) x_{1}^{2}\right]^{-1-\epsilon / 2}\right\} .
\end{aligned}
$$

Using this intermediate result we can apply the BT procedure to increment the power of the quadratic forms in the first and in the last integrals while the second one can be calculated analytically. Finally a Laurent expansion around $\epsilon=0$ is performed giving:

$$
\begin{aligned}
C_{0}^{\text {square }}= & \frac{1}{2} \sum_{i=1}^{4} \sum_{j=1}^{2} \sum_{l=1}^{2} \alpha_{i} \beta_{i} \zeta_{i j l}\left[C_{0, i j l}^{\text {square }}+\int d S_{1} C_{1, i j l}^{\text {square }}+\int d S_{2} C_{2, i j l}^{\text {square }}\right], \\
C_{0, i j l}^{\text {square }}= & \pi\left(h_{i j} \zeta_{i j l}^{2}\right)^{-1 / 2} b_{i j}^{-1 / 2} \ln \frac{1+\left(1+\kappa_{i j}\right)^{1 / 2}}{\kappa_{i j}^{1 / 2}} \\
& +\frac{1}{h_{i j} \zeta_{i j l}^{2}}\left[\ln B_{3}\left(1-\frac{1}{2} \ln P_{i j l}(1,0)\right)+\frac{1}{4} \ln ^{2} P_{i j l}(1,0)\right], \\
C_{1, i j l}^{\text {square }}= & \frac{1}{h_{i j} \zeta_{i j l}^{2}}\left[\frac{1}{2} \ln B_{3} \ln P_{i j l}(x, 0)-\frac{1}{4} \ln ^{2} \frac{P_{i j l}(x, 0)}{x^{2}}-\left(\frac{\ln P_{i j l}(1, x)}{x}\right)_{+}\right], \\
C_{2, i j l}^{\text {square }}= & \frac{1}{h_{i j} \zeta_{i j l}^{2}}\left(\frac{\ln P_{i j l}\left(x_{1}, x_{2}\right)}{x_{2}}\right)_{+}
\end{aligned}
$$

where we introduced the new quadratic form:

$$
P_{i j l}\left(x_{1}, x_{2}\right)=h_{i j} \zeta_{i j l}^{2}+b_{i j} x_{1}^{2}+B_{3} x_{2}^{2}-i \delta .
$$

Note that the poles in $\epsilon$ cancel out in Eq.(112), as expected. 


\section{Infrared divergent $C_{0}$}

Before discussing the general classification of IR divergent configurations for the three-point function, we consider a specific example, namely $p_{1}^{2}=-m_{1}^{2}, p_{2}^{2}=-m_{3}^{2}$ and $m_{2}=0$ (see Fig. 2). We obtain

$$
C_{0}^{I R} \equiv C_{0}\left(Q^{2},-m_{1}^{2},-m_{3}^{2} ; m_{1}, 0, m_{3}\right)=\int d C_{2} x_{1}^{-1-\epsilon} \chi^{-1-\epsilon / 2}\left(x_{2}\right)=-\frac{1}{\epsilon} \int_{0}^{1} d x \chi^{-1-\epsilon / 2}(x),
$$

where we introduced

$$
\chi(x)=-Q^{2} x^{2}+\left(Q^{2}+m_{3}^{2}-m_{1}^{2}\right) x+m_{1}^{2}, \quad Q^{2}=\left(p_{1}+p_{2}\right)^{2} .
$$

In this case the sector decomposition is trivial. The BT factor and co-factor associated to the quadratic form of Eq. (116) are

$$
B_{\mathrm{th}}=\frac{1}{4 Q^{2}} \lambda\left(-Q^{2}, m_{1}^{2}, m_{3}^{2}\right), \quad X=\frac{1}{2} \frac{Q^{2}+m_{3}^{2}-m_{1}^{2}}{Q^{2}} .
$$

and $B_{\mathrm{th}}=0$ at the normal(pseudo)-threshold of $C_{0}$. In the following we discuss the various cases under which Eq.115) can be classified.

\section{$5.1 \quad B_{\text {th }} \neq 0$}

As long as $B_{\text {th }} \neq 0$ it is possible to apply the standard BT procedure; we derive

$$
\begin{aligned}
C_{0}^{I R} & =-\frac{1}{B_{\mathrm{th}} \epsilon} \int_{0}^{1} d x\left[1+\frac{1}{\epsilon}(x-X) \frac{d}{d x}\right]\left[1-\frac{\epsilon}{2} \ln \chi(x)+\frac{\epsilon^{2}}{8} \ln ^{2} \chi(x)+\mathcal{O}\left(\epsilon^{3}\right)\right] \\
& =\frac{1}{B_{\mathrm{th}}}\left(\frac{C_{0}^{\mathrm{res}}}{\epsilon}+C_{0}^{\mathrm{fin}}+\mathcal{O}(\epsilon)\right),
\end{aligned}
$$

where the infrared residue and the finite part are

$$
\begin{aligned}
& C_{0}^{\mathrm{res}}=-1+\frac{1}{2}(1-X) \ln \chi(1)+\frac{1}{2} X \ln \chi(0)-\frac{1}{2} \int_{0}^{1} d x \ln \chi(x), \\
& C_{0}^{\mathrm{fin}}=-\frac{1}{8}(1-X) \ln ^{2} \chi(1)+\frac{1}{8} X \ln ^{2} \chi(0)+\frac{1}{2} \int_{0}^{1} d x\left[\ln \chi(x)+\frac{1}{4} \ln ^{2} \chi(x)\right] .
\end{aligned}
$$

\subsection{Infrared configuration and $B_{\text {th }} \approx 0$}

If $B_{\mathrm{th}} \approx 0$ then we introduce $h=-Q^{2}$ and write

$$
C_{0}^{I R}=-\frac{1}{\epsilon} \int_{-X}^{1-X} d x\left(h x^{2}+B_{\mathrm{th}}\right)^{-1-\epsilon / 2} .
$$

and consider three sub-cases: from Eq.(117) we see that $B_{\text {th }}=0$ only if $Q^{2}<0$, i.e. for $-Q^{2}=\left(m_{1} \pm m_{3}\right)^{2}$. Note that, in this case, we have $X=m_{1} /\left(m_{1} \pm m_{3}\right)$. 


\subsection{1 $0 \leq X \leq 1$}

Suppose that $0 \leq X \leq 1$ so that the configuration is actually singular at $B_{\text {th }}=0$. This corresponds to the normal threshold $Q^{2}=-\left(m_{1}+m_{3}\right)^{2}$. Introducing a Mellin-Barnes splitting, as described in Section 2 , and performing the $x$-integration gives

$$
\begin{aligned}
C_{0}^{I R} & =\frac{1}{2 \epsilon} \frac{1}{2 \pi i} \int_{-i \infty}^{+i \infty} d s \frac{\Gamma(-s) \Gamma(s+1+\epsilon / 2)}{\Gamma(1+\epsilon / 2)(s+1 / 2+\epsilon / 2)} \\
& \times(h-i \delta)^{-s-1-\epsilon / 2}\left(B_{\mathrm{th}}-i \delta\right)^{s}\left[(1-X)^{-1-2 s-\epsilon}+X^{-1-2 s-\epsilon}\right]
\end{aligned}
$$

with $-1-\epsilon / 2 \leq \operatorname{Re} s \leq-1 / 2-\epsilon / 2$. Note that we explicitly indicated the infinitesimal imaginary parts. Since we are interested in the behavior for $B_{\mathrm{th}} \rightarrow 0$ the $s$ contour can be closed over the right-hand complex half-plane at infinity, with poles at $s=-1 / 2-\epsilon / 2$ and at $s=k, k \geq 0$. The residue at $s=-1 / 2-\epsilon / 2$ is

$$
R\left(s=-\frac{1+\epsilon}{2}\right)=\frac{\pi}{h^{1 / 2}}\left(B_{\mathrm{th}}-i \delta\right)^{-1 / 2}\left\{-\frac{1}{\epsilon}+\frac{1}{2}\left[\ln \left(B_{\mathrm{th}}-i \delta\right)+\psi(1)-\psi\left(\frac{1}{2}\right)\right]\right\} .
$$

At $s=k$ we have instead

$$
\begin{aligned}
R(s=k) & =\frac{(-1)^{k}}{k+1 / 2} h^{-k-1} B_{\mathrm{th}}^{k} \sum_{a=X, 1-X} R(a) a^{-1-2 k} \\
R(a) & =\left\{\frac{1}{2 \epsilon}-\frac{1}{4}\left[\frac{1}{k+1 / 2}+\ln (h-i \delta)+2 \ln a+\psi(1)-\psi(k+1)\right]\right\} .
\end{aligned}
$$

Finally, we present the last two sub-cases.

\subsection{2 $X<0$ or $X>1$}

In these cases, corresponding to the pseudo-threshold $Q^{2}=-\left(m_{1}-m_{3}\right)^{2}$, we will write

$$
C_{0}^{I R}=-\frac{1}{\epsilon}[\theta(-X)-\theta(X-1)]\left[\int_{0}^{|1-X|} d x-\int_{0}^{|X|} d x\right]\left(h x^{2}+B_{\mathrm{th}}\right)^{-1-\epsilon / 2} .
$$

Suppose that $X<0$, after the Mellin-Barnes splitting we obtain

$$
\begin{aligned}
C_{0}^{I R} & =\frac{1}{2 \epsilon} \frac{1}{2 \pi i} \int_{-i \infty}^{+i \infty} d s \frac{\Gamma(-s) \Gamma(s+1+\epsilon / 2)}{\Gamma(1+\epsilon / 2)(s+1 / 2+\epsilon / 2)} \\
& \times(h-i \delta)^{-s-1-\epsilon / 2}\left(B_{\mathrm{th}}-i \delta\right)^{s}\left[(1-X)^{-1-2 s-\epsilon}-(-X)^{-1-2 s-\epsilon}\right] .
\end{aligned}
$$

There is no pole at $s=-1 / 2-\epsilon / 2$ but only poles at $s=k, k \geq 0$ with residues

$$
R(s=k)=\frac{(-1)^{k}}{k+1 / 2} h^{-k-1} B_{\mathrm{th}}^{k}\left[(1-X)^{-1-2 k} R(1-X)-|X|^{-1-2 k} R(|X|),\right.
$$

with a similar result if $X>1 . R$ is defined in Eq.(123). 


\subsection{IR configurations and Landau equations}

The study of the Landau equations for a given diagram is the most elegant way to classify its infrared divergent configurations. Again we refer to the one-loop vertex for illustration. The Landau equations are

$$
\begin{gathered}
\alpha_{1}\left(q^{2}+m_{1}^{2}\right)=0, \quad \alpha_{2}\left(\left(q+p_{1}\right)^{2}+m_{2}^{2}\right)=0, \quad \alpha_{3}\left(\left(q+p_{1}+p_{2}\right)^{2}+m_{3}^{2}\right)=0, \\
\alpha_{1} q_{\mu}+\alpha_{2}\left(q+p_{1}\right)_{\mu}+\alpha_{3}\left(q+p_{1}+p_{2}\right)_{\mu}=0 .
\end{gathered}
$$

A solution will be the leading singularity if it corresponds to $\alpha_{i} \neq 0, \forall i$. Next we multiply Eq.(128) by $q, p_{1}$ and $p_{2}$ respectively to obtain an homogeneous system of three equations where, moreover, we use $q^{2}=-m_{1}^{2}$ etc, from Eq.(127). A necessary and sufficient condition to have a proper solution, i.e. not all the $\alpha_{i}=0$, requires the determinant of coefficients to be zero, thus fixing a relation between internal and external masses. Any configuration that satisfy this constraint is a Landau singularity for the diagram which, however, does not necessarily imply that the diagram itself diverges at that configuration.

Suppose that we consider the following case: $p_{1,2}^{2}=-m^{2}, m_{1,3}=m$ and $m_{2}=0$. It is easily found that this configuration is a Landau singularity. The question is however of which kind. Let us insert the above values into the homogeneous system, what we obtain is

$$
m^{2} \alpha_{1}+\left(\frac{1}{2} Q^{2}+m^{2}\right) \alpha_{3}=0, \quad\left(\frac{1}{2} Q^{2}+m^{2}\right) \alpha_{1}+m^{2} \alpha_{3}=0 .
$$

First of all we observe that it is not either $\alpha_{1}=0$ or $q^{2}=-m_{1}^{2}$, etc; it can be both. Secondly, our configuration, where $Q^{2}=\left(p_{1}+p_{2}\right)^{2}$ is unconstrained, is a singularity. Finally there is a special case of the general configuration discussed $\left(Q^{2}\right.$ free) which is even more singular giving, in the infrared case, the true leading Landau singularity. To have $\alpha_{1,3} \neq 0$ we must require $Q^{2}=-4 \mathrm{~m}^{2}$ which gives, in the annihilation channel, the well-known threshold singularity on top of the infrared one. This condition emerges also from the following argument: inserting $p_{1,2}^{2}=-m^{2}$ in the condition to have a proper solution one obtains

$$
m_{2}^{2} Q^{2}=0, \quad \text { and } \quad m_{2}^{2}\left(m_{2}^{2}-Q^{2}-4 m^{2}\right)=0,
$$

In a certain sense the constraint $Q^{2}=-4 \mathrm{~m}^{2}$ is buried inside the anomalous threshold condition. In other words, that all the propagators in a diagram are on-shell and that the consistency relation is satisfied does not necessarily imply that all $\alpha_{i}$ are different from zero: the infrared case is a clear example. Note the presence of a potential singularity also at $Q^{2}=0$ which, however, is not physical, i.e. not on the physical Riemann sheet. The latter fact can be seen by inspecting the explicit analytic result,

$$
C_{0}^{I R}=\frac{2}{\beta Q^{2}} \ln \frac{\beta+1}{\beta-1} \frac{1}{\epsilon}+\text { IR finite, } \quad \beta^{2}=1+4 \frac{m^{2}}{Q^{2}} .
$$

Our strategy in the general classification of infrared divergent configurations, diagram by diagram, will be to assume a certain number of zero internal masses with at least one unconstrained external momentum. Then we fix the remaining parameters to satisfy the consistency relation for the Landau equations. Finally, we return to the original set of Landau equations and look for additional constraints that are necessary in order to arrive to the true leading singularity. The presence of a threshold-like singularity on top of the infrared poles is the sign that, after extracting these poles, we still have complications for the residual integrations that cannot be solved with naive methods.

For $C_{0}$ and arbitrary masses we look for a solution of the homogeneous system of the three Landau equations in a situation where $Q^{2}$ is not constrained (due to the symmetry of the problem it is sufficient 
to consider $Q^{2}$ ). The condition to have a proper solution is a quadratic equation in $Q^{2}$ with $m_{2}^{2}$ as the coefficient of $Q^{4}$; therefore we require $m_{2}=0$, after which we obtain the following condition:

$$
\left(p_{1}^{2}+m_{1}^{2}\right)\left(p_{2}^{2}+m_{3}^{2}\right) Q^{2}-\left[\left(p_{1}^{2}+m_{1}^{2}\right)-\left(p_{2}^{2}+m_{3}^{2}\right)\right]\left[m_{1}^{2}\left(p_{2}^{2}+m_{3}^{2}\right)-m_{3}^{2}\left(p_{1}^{2}+m_{1}^{2}\right)\right]=0,
$$

requiring $p_{1}^{2}=-m_{1}^{2}$ and $p_{2}^{2}=-m_{3}^{2}$, i.e. the configuration that we already discussed. If we substitute back into the Landau equations we obtain

$$
\begin{aligned}
& 2 m_{1}^{2} \alpha_{1}+\left(Q^{2}+m_{1}^{2}+m_{3}^{2}\right) \alpha_{3}=0, \\
& \left(Q^{2}+m_{1}^{2}+m_{3}^{2}\right) \alpha_{1}+2 m_{3}^{2} \alpha_{3}=0,
\end{aligned}
$$

so that the leading singularity, i.e. all $\alpha$ 's different from zero, is obtained for

$$
\lambda\left(-Q^{2}, m_{1}^{2}, m_{3}^{2}\right)=0 \quad \equiv \quad B_{3}=0,
$$

which clarifies once more the physical meaning of the BT factor.

\section{Four-point functions (D-family)}

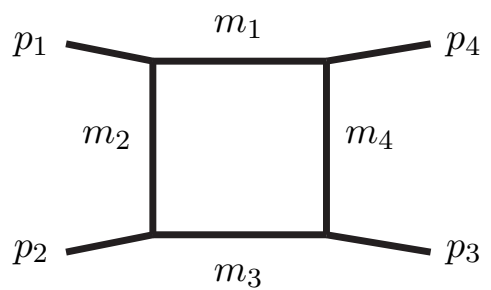

Figure 3: The one-loop, four-point Green function of Eq.(135). All momenta are flowing inwards.

The generic, scalar, box (see Fig. 3) is given by

$$
D_{0}=\int d S_{3} V^{-2-\epsilon / 2}\left(x_{1}, x_{2}, x_{3}\right), \quad V(x)=x^{t} H x+2 K^{t} x+L,
$$

where $H_{i j}=-p_{i} \cdot p_{j}, L=m_{1}^{2}$ and where

$$
\begin{aligned}
& K_{1}=-\frac{1}{2}\left(m_{1}^{2}-m_{2}^{2}-p_{1} \cdot p_{1}\right), \quad K_{2}=-\frac{1}{2}\left(m_{2}^{2}-m_{3}^{2}-2 p_{1} \cdot p_{2}-p_{2} \cdot p_{2}\right), \\
& K_{3}=-\frac{1}{2}\left(m_{3}^{2}-m_{4}^{2}-2 p_{1} \cdot p_{3}-2 p_{2} \cdot p_{3}-p_{3} \cdot p_{3}\right) .
\end{aligned}
$$

We are now in a position to write the answer for the case $B_{4} \neq 0$.

\subsection{Evaluation of $D_{0}$ when $B_{4} \neq 0$}

If we are away from $B_{4}=L-K^{t} H^{-1} K=0$ we can apply a BT iteration obtaining

$$
B_{4} D_{0}=-\frac{1}{2} \int d S_{3} V^{-1-\epsilon / 2}\left(x_{1}, x_{2}, x_{3}\right)+\frac{1}{2} \int d S_{2} \sum_{i=0}^{3}\left(X_{i}-X_{i+1}\right) V^{-1-\epsilon / 2}(\widehat{i+1+1}),
$$


where $X=-K^{t} H^{-1}$ and, moreover, $X_{0}=1, X_{4}=0$. Contractions are defined as before with

$$
\widehat{01}=\left(1, x_{1}, x_{2}\right), \quad \widehat{34}=\left(x_{1}, x_{2}, 0\right),
$$

etc. Therefore $D_{0}(d=4)$ is a combination of a non-contracted term which can be seen as $D_{0}(d=6)$ and of four pinches which can be represented as the triangles arising when we shrink a line of the box to a point. The latter are three-point functions that can be treated according to the results of Section 4 . Furthermore, if $B_{4} \neq 0, D_{0}(d=6)$ can be treated with a second BT iteration giving rise to logarithms,

$$
\begin{aligned}
B_{4} D_{0}(d=6) & =\int d S_{3} V^{-1-\epsilon / 2}\left(x_{1}, x_{2}, x_{3}\right)=\int d S_{3}\left[\frac{3}{2} \ln V\left(x_{1}, x_{2}, x_{3}\right)+1\right] \\
& -\frac{1}{2} \int d S_{2} \sum_{i=0}^{3}\left(X_{i}-X_{i+1}\right) \ln V(\widehat{i i+i}) .
\end{aligned}
$$

Note that each three-point function will have, after the second iteration, its own sub-leading BT coefficient. As long as $B_{4} \neq 0$, the case where some or all of the sub-leading $B$ are zero is fully covered by the results of Section 1 . Similarly to Eq.(36), the case of $G_{4}=\operatorname{det}(H)=0$ reduces $D_{0}$ to a combination of four $C_{0}$ functions.

\subsection{Form factors in the D-family}

Also form factors can be written in a compact way by using the functions

$$
\begin{aligned}
\mathcal{D}_{n_{1}, n_{2}, n_{3}} & =\int d S_{3} x_{1}^{n_{1}} x_{2}^{n_{2}} x_{3}^{n_{3}} V^{-2-\epsilon / 2}\left(x_{1}, x_{2}, x_{3}\right), \\
\mathcal{C}_{i}(\widehat{j j+1}) & =\int d S_{2} x_{i} V^{-1-\epsilon / 2}(\widehat{j j+1}) .
\end{aligned}
$$

Here we give one explicit example,

$$
B_{4} \mathcal{D}_{100}=\frac{1}{2} X_{1} D_{0}(d=6)-\mathcal{D}_{100}(d=6)+\frac{1}{2}\left[\left(1-X_{1}\right) C_{0}(\widehat{01})+\sum_{i=1}^{3}\left(X_{i}-X_{i+1}\right) \mathcal{C}_{1}(\widehat{i i+1})\right]
$$

where the $d=6$ component gives

$$
\begin{aligned}
B_{4} \mathcal{D}_{100}(d=6) & =\int d S_{3}\left[\left(2 x_{1}-\frac{1}{2} X_{1}\right) \ln V\left(x_{1}, x_{2}, x_{3}\right)+x_{1}\right] \\
& -\frac{1}{2} \int d S_{2}\left[\left(1-X_{1}\right) \ln V(\widehat{01})+\sum_{i=1}^{3}\left(X_{i}-X_{i+1}\right) x_{1} \ln V(\widehat{i i+1})\right] .
\end{aligned}
$$

Suppose now that $B_{4} \approx 0$ : an alternative derivation for $D_{0}$ is needed. However, we first consider an extension of Eq.(139). 


\section{3 $\quad D_{0}$ at $\mathcal{O}(\epsilon)$}

A three-point function with the insertion of a counterterm, which is needed in a two-loop calculation, is formally identical to a one-loop four-point function with one zero external momentum. Its expression at $\mathcal{O}(\epsilon)$ is needed and this follows from the BT algorithm quite nicely,

$$
D_{0}(d)=D_{0}(4)+\epsilon D_{0}^{(1)}+\mathcal{O}\left(\epsilon^{2}\right) .
$$

We obtain

$$
16 B_{4} D_{0}^{(1)}=\frac{1}{B_{4}} \int d S_{3} I_{3}+\int d S_{2}\left[\frac{I_{2}^{A}}{B_{4}}-2 \sum_{i=w, s, e, n} \frac{d_{i} I_{2 i}^{B}}{B_{3 i}}\right]+\sum_{i=w, s, e, n} \sum_{j=r, g, b} \frac{d_{i} d_{i j}}{B_{3 i}} \int d S_{1} I_{1 i j},
$$

where the integrand is given by

$$
\begin{gathered}
I_{3}=2+22 \ln V\left(x_{1}, x_{2}, x_{3}\right)+3 \ln ^{2} V\left(x_{1}, x_{2}, x_{3}\right), \\
I_{2}^{A}=-\sum_{i=w, s, e, n} d_{i}\left[6 \ln V_{i}\left(x_{1}, x_{2}\right)+\ln ^{2} V_{i}\left(x_{1}, x_{2}\right)\right], \\
I_{2 i}^{B}=1+4 \ln V_{i}\left(x_{1}, x_{2}\right)+\ln ^{2} V_{i}\left(x_{1}, x_{2}\right), \quad I_{1 i j}=2 \ln V_{i j}\left(x_{1}\right)+\ln ^{2} V_{i j}\left(x_{1}\right),
\end{gathered}
$$

where we introduced the quadratics

$$
\begin{gathered}
V_{w}=V\left(x_{1}, x_{1}, x_{2}\right), \quad V_{s}=V\left(x_{1}, x_{2}, x_{2}\right), \quad V_{e}=V\left(x_{1}, x_{2}, 0\right), \quad V_{n}=V\left(1, x_{1}, x_{2}\right), \\
V_{i r}=V_{i}\left(x_{1}, x_{1}\right), \quad V_{i g}=V_{i}\left(x_{1}, 0\right), \quad V_{i b}=V_{i}\left(1, x_{1}\right),
\end{gathered}
$$

and the auxiliary factors

$$
\begin{gathered}
d_{w}=X_{1}-X_{2}, \quad d_{s}=X_{2}-X_{3}, \quad d_{e}=X_{3}, \quad d_{n}=1-X_{1}, \\
d_{i r}=X_{i 1}-X_{i 2}, \quad d_{i g}=X_{i 2} \quad d_{i b}=1-X_{i 1} .
\end{gathered}
$$

In the last equation $X_{i}$ and $X_{i j}$ are the BT co-factors of the corresponding quadratics defined by Eqs. (148)-1149).

\subsection{Evaluation of $D_{0}$ when $B_{4} \approx 0$ and $D_{0}$ is regular}

This is the case when $B_{4} \rightarrow 0$ but the condition $0<X_{3}<X_{2}<X_{1}<1$ is not fulfilled, i.e. the point of coordinates $X_{i}$ is outside the original simplex. Thus $D_{0}$ is regular and the integration hyper-contour can be distorted to avoid the zeros of $V$ without pinches. Two methods will be introduced to cover this case. 


\subsubsection{Method I}

We proceed analogously to the case of $C_{0}$. First of all we define $V_{0}\left(x_{1}, x_{2}, x_{3}\right)=V\left(x_{1}, x_{2}, x_{3}\right)-B_{4}$ and write down the BT relations corresponding to $B_{4} \neq 0$ and $B_{4}=0$ :

$$
\begin{aligned}
& {\left[1+\frac{1}{2+\epsilon}(x-X) \partial_{x}\right] V^{-1-\epsilon / 2}\left(x_{1}, x_{2}, x_{3}\right)=B_{4} V^{-2-\epsilon / 2}\left(x_{1}, x_{2}, x_{3}\right),} \\
& {\left[1+\frac{1}{2+\epsilon}(x-X) \partial_{x}\right] V_{0}^{-1-\epsilon / 2}\left(x_{1}, x_{2}, x_{3}\right)=0 .}
\end{aligned}
$$

Then we sum each side of the two equations and integrate by parts, obtaining:

$$
D_{0}=-\left.\frac{1}{2} \int d S_{3} B_{4}^{-1} V^{-1-\epsilon / 2}\left(x_{1}, x_{2}, x_{3}\right)\right|_{\mathrm{sub}}+\left.\frac{1}{2} \int d S_{2} \sum_{i=0}^{3}\left(X_{i}-X_{i+1}\right) B_{4}^{-1} V^{-1-\epsilon / 2}(\widehat{i i+1})\right|_{\mathrm{sub}}
$$

where $\left.V^{\mu}\right|_{\text {sub }}=V^{\mu}-V_{0}^{\mu}$. The twofold integrals are nothing but differences of three-point functions that can be easily treated according to the results obtained for $C_{0}$ in Section 1 . For the first integral of Eq.(153) we set $\epsilon=0$ and apply again the BT algorithms:

$$
\begin{aligned}
1-\frac{1}{2}(x-X) \partial_{x} \ln V\left(x_{1}, x_{2}, x_{3}\right) & =B_{4} V^{-1}\left(x_{1}, x_{2}, x_{3}\right), \\
B_{4}\left[1+\frac{1}{2}(x-X) \partial_{x}\right] V_{0}^{-1}\left(x_{1}, x_{2}, x_{3}\right) & =0 \\
1-\frac{1}{2}(x-X) \partial_{x} \ln V_{0}\left(x_{1}, x_{2}, x_{3}\right) & =0 .
\end{aligned}
$$

By summing the first two equations and by subtracting the last one, we get:

$$
\left.B_{4}^{-1} V^{-1}\left(x_{1}, x_{2}, x_{3}\right)\right|_{\mathrm{sub}}=-\frac{1}{2}(x-X) \partial_{x}\left(\frac{\ln V\left(x_{1}, x_{2}, x_{3}\right)}{B_{4}^{2}}\right)_{++},
$$

where we introduced the ' ++ distribution'

$$
\left(\frac{\ln V\left(x_{1}, x_{2}, x_{3}\right)}{B_{4}^{2}}\right)_{++} \equiv \frac{\ln V\left(x_{1}, x_{2}, x_{3}\right)-\ln V_{0}\left(x_{1}, x_{2}, x_{3}\right)-B_{4} V_{0}^{-1}\left(x_{1}, x_{2}, x_{3}\right)}{B_{4}^{2}} .
$$

Now another integration by parts is performed and we obtain:

$$
\begin{gathered}
\left.\int d S_{3} B_{4}^{-1} V^{-1}\left(x_{1}, x_{2}, x_{3}\right)\right|_{\mathrm{sub}}=\frac{3}{2} \int d S_{3}\left(\frac{\ln V\left(x_{1}, x_{2}, x_{3}\right)}{B_{4}^{2}}\right)_{++} \\
-\frac{1}{2} \int d S_{2} \sum_{i=0}^{3}\left(X_{i}-X_{i+1}\right)\left(\frac{\ln V(\widehat{i+1+1})}{B_{4}^{2}}\right)_{++} .
\end{gathered}
$$

In the twofold integral the ' ++ ' distribution contains terms such as $V_{0}^{-1}(\widehat{i i+1})$ which are three-point functions with $\epsilon=0$ and can be calculated in the usual way. In the first integral we have $V_{0}^{-1}\left(x_{1}, x_{2}, x_{3}\right)$ which can be reduced again to a sum of three-point functions:

$$
\int d S_{3} V_{0}^{-1}\left(x_{1}, x_{2}, x_{3}\right)=\int d S_{2} \sum_{i=0}^{3}\left(X_{i}-X_{i+1}\right) V_{0}^{-1}(\widehat{i+1+1}) .
$$

This result is obtained by using the second relation of Eq.(154) together with an integration by parts. An analogous procedure can be derived for tensor integrals. 


\subsubsection{Method II}

Also for the four-point function another method is available. First of all we perform a Taylor expansion around $B_{4}=0$ in Eq.(135):

$$
D_{0}=\int d S_{3}(X)\left[x^{t} H x+B_{4}\right]^{-2-\epsilon / 2}=\sum_{n=0}^{\infty}(n+1) \mathcal{D}(n+2)\left(-B_{4}\right)^{n},
$$

and then we use the BT relation

$$
\left[1+\frac{x \partial_{x}}{2 n+\epsilon}\right]\left(x^{t} H x\right)^{-n-\epsilon / 2}=0 .
$$

In this way, an arbitrary coefficient in the Taylor expansion in $B_{4}$ can be reduced to a twofold integral to be treated according to a standard BT algorithm. There will be several new quadratic forms to be introduced, in two and one variables. We obtain the following form for the coefficients

$$
\mathcal{D}(n)=\int d S_{3}(X)\left(x^{t} H x\right)^{-n-\epsilon / 2}=\frac{1}{3-2 n} \sum_{i=0}^{3} X_{i}^{L} \int d S_{2} Q^{-n-\epsilon / 2}(T \widehat{i+1+1}),
$$

where $X_{i}^{L}=X_{i}-X_{i+1}$ and the quadratic forms $Q(T \widehat{i i+1})$ are

$$
\begin{aligned}
& Q(T \widehat{011}) \equiv Q_{0}=Q\left(1-X_{1}, x_{1}-X_{2}, x_{2}-X_{3}\right), \\
& Q(T \widehat{12}) \equiv Q_{1}=Q\left(x_{1}-X_{1}, x_{1}-X_{2}, x_{2}-X_{3}\right), \\
& Q(T \widehat{23}) \equiv Q_{2}=Q\left(x_{1}-X_{1}, x_{2}-X_{2}, x_{2}-X_{3}\right), \\
& Q(T \widehat{34}) \equiv Q_{3}=Q\left(x_{1}-X_{1}, x_{2}-X_{2},-X_{3}\right) .
\end{aligned}
$$

In general we will write $Q_{i}=x^{t} H_{i} x+2 K_{i}^{t} x+L_{i}$ and introduce corresponding sub-leading BT factors $B_{i}=L_{i}-K_{i}^{t} H_{i}^{-1} K_{i}$ and co-factors $X_{i}=-K_{i}^{t} H_{i}^{-1}$ with element $X_{i j}(j=1,2)$. A second iteration will bring into the result quadratic forms in one variable,

$$
Q_{i, 0}(x)=Q_{i}(1, x), \quad Q_{i, 1}(x)=Q_{i}(x, x), \quad Q_{i, 2}(x)=Q_{i}(x, 0),
$$

which are written as $Q_{i j}(x)=h_{i j} x^{2}+2 k_{i j} x+l_{i j}$ and also have a sub-sub-leading BT factor $b_{i j}=$ $l_{i j}-k_{i j}^{2} / h_{i j}$ and a co-factor $X_{i j}$. By applying again the BT procedure we finally obtain for $\mathcal{D}(2)$

$$
\begin{gathered}
\mathcal{D}(2)=\frac{1}{4} \sum_{i=0}^{3} \sum_{j=0}^{2} \frac{X_{i}^{L} X_{i j}^{L}}{B_{i} b_{i j}}\left[\mathcal{D}_{0, i j}(2)+\int_{0}^{1} d x \mathcal{D}_{1, i j}(2)\right], \\
\mathcal{D}_{0, i j}(2)=\left(1+\frac{k_{i j}}{h_{i j}}\right) \ln Q_{i j}(1)-\frac{k_{i j}}{h_{i j}} \ln Q_{i j}(0)-2, \quad \mathcal{D}_{1, i j}(2)=-\ln Q_{i j}(x),
\end{gathered}
$$

while for $n \neq 2$

$$
\begin{gathered}
\mathcal{D}(n)=\frac{1}{(3-2 n)(n-1)} \sum_{i=0}^{3} \frac{X_{i}^{L}}{B_{i}}\left[\mathcal{D}_{0, i}(n)+\int_{0}^{1} d x \mathcal{D}_{1, i}(n)+\int d S_{2} \mathcal{D}_{2, i}(n)\right] \\
\mathcal{D}_{0, i}(n)=\frac{1}{4(n-2)} \sum_{j=0}^{2} \frac{X_{i j}^{L}}{b_{i j}}\left[\left(1+\frac{k_{i j}}{h_{i j}}\right) Q_{i j}^{-n+2}(1)-\frac{k_{i j}}{h_{i j}} Q_{i j}^{-n+2}(0)\right]
\end{gathered}
$$




$$
\mathcal{D}_{1, i}(n)=\frac{1}{4(n-2)} \sum_{j=0}^{2} X_{i j}^{L}\left(\frac{2(n-2)}{B_{i}}+\frac{2 n-5}{b_{i j}}\right) Q_{i j}^{-n+2}(x), \quad \mathcal{D}_{2, i}(n)=\frac{n-3}{B_{i}} Q_{i}^{-n+2}\left(x_{1}, x_{2}\right) .
$$

Now we can sum the series according to well-known results:

$$
\sum_{n=1}^{\infty} x^{n}=\frac{x}{1-x}, \quad \sum_{n=1}^{\infty} \frac{x^{n}}{n}=-\ln (1-x), \quad \sum_{n=1}^{\infty} \frac{x^{n}}{(2 n+1) n}=-\int_{0}^{1} d y \ln \left(1-x y^{2}\right),
$$

and obtain:

$$
D_{0}=\mathcal{D}(2)+\sum_{i=0}^{3} \frac{X_{i}^{L}}{B_{i}}\left[D_{0, i}+\int_{0}^{1} d x D_{1, i}+\int d S_{2} D_{2, i}\right]
$$

where

$$
\begin{aligned}
D_{0, i} & =\frac{1}{4} \sum_{j=0}^{2} \frac{X_{i j}^{L}}{b_{i j}} \int_{0}^{1} d y\left[\left(1+\frac{k_{i j}}{h_{i j}}\right) \ln \frac{Q_{i j}(1)+B_{4} y^{2}}{Q_{i j}(1)}-\frac{k_{i j}}{h_{i j}} \ln \frac{Q_{i j}(0)+B_{4} y^{2}}{Q_{i j}(0)}\right], \\
D_{1, i} & =\frac{1}{4} \sum_{j=0}^{2} X_{i j}^{L}\left[\left(\frac{1}{B_{i}}+\frac{1}{b_{i j}}\right) \ln \frac{Q_{i j}(x)+B_{4}}{Q_{i j}(x)}-\left(\frac{1}{B_{i}}+\frac{2}{b_{i j}}\right) \int_{0}^{1} d y \ln \frac{Q_{i j}(x)+B_{4} y^{2}}{Q_{i j}(x)}\right], \\
D_{2, i}(n) & =\frac{1}{2 B_{i}}\left[\frac{B_{4}}{Q_{i}\left(x_{1}, x_{2}\right)+B_{4}}-\frac{3}{2} \ln \frac{Q_{i}\left(x_{1}, x_{2}\right)+B_{4}}{Q_{i}\left(x_{1}, x_{2}\right)}+\frac{3}{2} \int_{0}^{1} d y \ln \frac{Q_{i}\left(x_{1}, x_{2}\right)+B_{4} y^{2}}{Q_{i}\left(x_{1}, x_{2}\right)}\right] .
\end{aligned}
$$

In the twofold integral we still have a polynomial with a negative power; it is possible to increment the power of the latter by employing the BT relation:

$$
\left(B_{i}+B_{4}\right)\left(Q_{i}+B_{4}\right)^{-1}=1-\frac{1}{2}\left(x-X_{i}\right) \partial_{x} \ln \left(Q_{i}+B_{4}\right) .
$$

The usual integration by parts gives:

$$
\int d S_{2}\left(Q_{i}+B_{4}\right)^{-1}=\frac{1}{2\left(B_{i}+B_{4}\right)}\left[1+2 \int d S_{2} \ln \left(Q_{i}+B_{4}\right)-\sum_{j=0}^{2} X_{i j}^{L} \int d S_{1} \ln \left(Q_{i j}+B_{4}\right)\right] .
$$

In this way all coefficients in Eq. (160) are explicitly computed. We can treat form factors with the same procedure; indeed the auxiliary functions

$$
D(\{n\})=\int d S_{3} \prod_{i=1}^{3} x_{i}^{n_{i}} V^{-2-\epsilon / 2}\left(x_{1}, x_{2}, x_{3}\right),
$$

become linear combinations of

$$
\begin{aligned}
\mathcal{D}\left(n, n_{1}, n_{2}, n_{3}\right) & =\frac{1}{3-2 n+n_{1}+n_{2}+n_{3}} \int d S_{2} \\
& \times\left\{\left(x_{1}-X_{2}\right)^{n_{2}}\left(x_{2}-X_{3}\right)^{n_{3}}\left(1-X_{1}\right)^{n_{1}+1} Q_{0}^{-n}\left(x_{1}, x_{2}\right)\right. \\
& +\left(x_{1}-X_{1}\right)^{n_{1}}\left(x_{1}-X_{2}\right)^{n_{2}}\left(x_{2}-X_{3}\right)^{n_{3}}\left(X_{1}-X_{2}\right) Q_{1}^{-n}\left(x_{1}, x_{2}\right) \\
& +\left(x_{2}-X_{2}\right)^{n_{2}}\left(x_{2}-X_{3}\right)^{n_{3}}\left(x_{1}-X_{1}\right)^{n_{1}}\left(X_{2}-X_{3}\right) Q_{2}^{-n}\left(x_{1}, x_{2}\right) \\
& \left.-\left(x_{1}-X_{1}\right)^{n_{1}}\left(x_{2}-X_{2}\right)^{n_{2}}\left(-X_{3}\right)^{n_{3}+1} Q_{3}^{-n}\left(x_{1}, x_{2}\right)\right\},
\end{aligned}
$$

which are form factors of the $C$-family. 


\subsubsection{Sub-leading behavior}

The above derivation assumes that the sub-leading BT factors are not zero. If this is the case we start from the fact that $D_{0}$ is a sum of terms of the form

$$
D_{0}^{i}=-\sum_{n=0}^{\infty} \frac{n+1}{2 n+1}\left(-B_{4}\right)^{n} \int d S_{2} q_{i} Q_{i}^{-n-2},
$$

with $q_{i}$ constant. When $B_{i} \approx 0$ we cannot apply the BT algorithm and it is necessary to adopt an alternative strategy. If the generalized three-point function associated to the vanishing coefficient $B_{i}$ is regular, we can perform another Taylor expansion exactly as it was done for $C_{0}$. Otherwise we rewrite the sum as follows:

$$
\sum_{n=0}^{\infty} \frac{n+1}{2 n+1} Q_{i}^{-n-2}\left(-B_{4}\right)^{n}=\left(B_{4}\right)^{-1 / 2} \int_{0}^{\sqrt{B_{4}}} d z\left(Q_{i}+z^{2}\right)^{-2} .
$$

To the price of introducing an extra integration we are now in the situation where the series is replaced by a single term and the sub-leading BT factors for the $x_{1}-x_{2}$ integration are replaced by $B_{i}+z^{2}$ and the singular behavior is left in the last $z$ integration. Note that, if $B_{4}<0$ we simply have to replace everywhere $B_{4}$ and $Q_{i}$ with $-B_{4},-Q_{i}$. Actually the best way of dealing with the singular behavior is to change variable and obtain

$$
\int d S_{2}(X) \int_{0}^{1} d z\left(x^{t} H_{i} x+B_{3 i}+B_{4} z^{2}\right)^{-2}
$$

and perform a Mellin-Barnes splitting (accompanied by a sector decomposition) of $x^{t} H_{i} x$ and $B_{3 i}+B_{4} z^{2}$. The leading behavior comes from the single pole at $s=1$ and it is, therefore, proportional to

$$
\left(B_{4} B_{3 i}\right)^{-1 / 2} \arctan \left(\frac{B_{4}}{B_{3 i}}\right)^{1 / 2}
$$

where $B \rightarrow B-i \delta$. Using the identity of Eq.(178) in Eq.(177) we obtain

$$
D_{0}^{i}=-q_{i} \int_{0}^{1} d z \int d S_{2}\left(Q_{i}+B_{4} z^{2}\right)^{-2}
$$

The first step toward the evaluation of the integral consists in splitting the integral in the sum of two parts:

$$
D_{0}^{i} \equiv I_{1}^{i}-I_{2}^{i}, \quad I_{1 ; 2}^{i}=-q_{i} \int_{0}^{1} d z \int_{0}^{1} d x_{1} \int_{0 ; x_{1}}^{1} d x_{2}\left(Q_{i}+B_{4} z^{2}\right)^{-2} .
$$

Now the first integral is singular when $B_{3 i} \rightarrow 0$ while the second is not. Next, we consider the calculation of $I_{1}^{i}$. Introducing the new variables of integration $x_{i}^{\prime}=x_{i}-X_{i}$, we have that

$$
I_{1}^{i}=-q_{i} \int d C_{2}(X) \int_{0}^{1} d z\left(x^{t} H x+B_{3 i}+B_{4} z^{2}\right)^{-2}
$$

then we apply the Mellin-Barnes transform and obtain

$$
I_{1}^{i}=-\frac{q_{i}}{2 \pi i} \int d C_{2}(X) \int_{0}^{1} d z \int_{-i \infty}^{i \infty} d s B(s, 2-s)\left(x^{t} H x\right)^{-s} \rho_{\text {mix }}^{2-s},
$$


where $1 / \rho_{\text {mix }}=B_{4} z^{2}+B_{3 i}$. It is possible to perform the integration over the variables $x_{i}$ and the result can be cast into the following, general, form:

$$
\int d C_{2}(X)\left(x^{t} H x\right)^{-s}=\frac{f(s)}{s-1} .
$$

As a consequence we obtain

$$
I_{1}^{i}=-\frac{q_{i}}{2 \pi i} \int_{0}^{1} d z \int_{-i \infty}^{i \infty} d s B(s, 2-s) \rho_{\operatorname{mix}}^{2-s} \frac{f(s)}{s-1} .
$$

We can now perform the integration over $s$ and the leading divergent behavior is given by the pole at $s=1$ :

$$
I_{1}^{i, \text { sing }}=q_{i}\left(B_{4} B_{3 i}\right)^{-1 / 2} f(1) \arctan \left(\frac{B_{4}}{B_{3 i}}\right)^{1 / 2}
$$

where the determination of the arctan function is fixed by the $-i \delta$ prescription. The regular part of the integral $I_{1}^{i}$ is

$$
I_{1}^{i, \mathrm{reg}}=-q_{i} \sum_{n=0}^{\infty}(-1)^{n} \int_{0}^{1} d z f(n+2)\left(B_{4} z^{2}+B_{3 i}\right)^{n},
$$

the integration over $z$ can then be performed:

$$
I_{1}^{i, \text { reg }}=q_{i} \sum_{n=0}^{\infty}(-1)^{n+1} f(n+2)\left(B_{3 i}\right)^{n}{ }_{2} F_{1}\left(\frac{1}{2},-n ; \frac{3}{2} ;-\frac{B_{4}}{B_{3 i}}\right) .
$$

The integral $I_{2}$ can be calculated by performing a Taylor expansion around $B_{4} z^{2}+B_{3 i}=0$ :

$$
\begin{aligned}
I_{2}^{i} & =-q_{i} \sum_{k=0}^{\infty} \int_{0}^{1} d z\left(-B_{4} z^{2}-B_{3 i}\right)^{k} \int_{0}^{1} d x_{1} \int_{x_{1}}^{1} d x_{2}(k+1)\left[(x-X)^{t} H_{i}(x-X)\right]^{-2-k}, \\
& =-q_{i} \sum_{k=0}^{\infty}\left(-B_{3 i}\right)^{k}(k+1)_{2} F_{1}\left(\frac{1}{2},-n ; \frac{3}{2} ;-\frac{B_{4}}{B_{3 i}}\right) \mathcal{I}_{2}(n+2),
\end{aligned}
$$

where we introduced the integral $\mathcal{I}_{2}(n)$ defined by

$$
\mathcal{I}_{2}(n)=\int_{0}^{1} d x_{1} \int_{x_{1}}^{1} d x_{2}\left[(x-X)^{t} H_{i}(x-X)\right]^{-n}=\int d \bar{S}_{2}(X)\left[x^{t} H_{i} x\right]^{-n} .
$$

These integrals can be treated in analogy to what was done for $\mathcal{C}(n)$ (Eq.(52)) and $\mathcal{D}(n)$ (Eq.(162)); using the relation

$$
\left[1+\frac{x \partial}{2 n}\right]\left(x^{t} H x\right)^{-n}=0
$$

we obtain

$$
\mathcal{I}_{2}(n)=\frac{1}{2(n-1)} \int_{0}^{1} d x\left[\left(1-X_{2}\right) \mathcal{P}_{1}^{-n}+\left(X_{2}-X_{1}\right) \mathcal{P}_{2}^{-n}+X_{1} \mathcal{P}_{3}^{-n}\right]
$$

where $\mathcal{P}\left(x_{1}, x_{2}\right)=x^{t} H x$ and where

$$
\mathcal{P}_{1}=\mathcal{P}\left(1-X_{2}, x-X_{1}\right), \quad \mathcal{P}_{2}=\mathcal{P}\left(x-X_{2}, x-X_{1}\right), \quad \mathcal{P}_{3}=\mathcal{P}\left(x-X_{2}, X_{1}\right) .
$$

The remaining one-dimensional integrals are now generalized $B_{0}$ functions which can be calculated employing the methods introduced in Section 3 . 


\subsection{Evaluation of $D_{0}$ when $B_{4} \approx 0$ and $D_{0}$ is singular}

This is the case when $B_{4} \rightarrow 0$ and the condition $0<X_{3}<X_{2}<X_{1}<1$ is fulfilled, i.e. the point of coordinates $X_{i}$ is inside the original simplex. Thus $D_{0}$ is singular and the integration hyper-contour cannot be distorted due to the occurrence of a pinch. In order to minimize the number of terms in the solution we write

$$
\int_{0}^{1} \prod_{i=1}^{3} d x_{i}=\sum_{\text {cycl.perm. }} \int_{0}^{1} d x_{i} \int_{0}^{x_{i}} d x_{j} \int_{0}^{x_{i}} d x_{k}
$$

Furthermore, for each term we may use the following identity

$$
\int_{0}^{x_{1}} d x_{2} \int_{0}^{x_{1}} d x_{3}=\int_{0}^{x_{1}} d x_{2} \int_{0}^{x_{2}} d x_{3}+\int_{0}^{x_{1}} d x_{3} \int_{0}^{x_{3}} d x_{2} .
$$

Therefore the integral over $[0,1]^{3}$ can be written as

$$
\int_{0}^{1} \prod_{i=1}^{3} d x_{i} V^{-2-\epsilon / 2}\left(x_{1}, x_{2}, x_{3}\right)=\int d S_{3} \sum_{\text {perm }} V^{-2-\epsilon / 2}\left(x_{1}, x_{2}, x_{3}\right),
$$

where the sum is over all permutations of $\left\{x_{1}, x_{2}, x_{3}\right\}$. The first term in the sum is exactly our original $D_{0}$ function while the rest gives the five complementary functions which, by construction, are regular and can be treated according to the strategy of Section 6.4. As a consequence, we now have to evaluate $D_{0}^{\text {cube }}$ when $B_{4} \approx 0$ and the point of coordinates $X_{i}$ is inside the unit cube. Using standard techniques, already applied to the $C_{0}$-function, we obtain

$$
\begin{aligned}
D_{0}^{\text {cube }} & =\left\{\prod_{i=1}^{3} \int_{-X_{i}}^{1-X_{i}} d x_{i}\right\}\left(x^{t} H x+B_{4}\right)^{-2-\epsilon / 2} \\
& =\sum_{i=1}^{8} \int_{0}^{\alpha_{i}} d x_{1} \int_{0}^{\beta_{i}} d x_{2} \int_{0}^{\gamma_{i}} d x_{3}\left(Q_{i}+B_{4}\right)^{-2-\epsilon / 2} .
\end{aligned}
$$

If $Q\left(x_{1}, x_{2}, x_{3}\right)=x^{t} H x$ the quadrics $Q_{i}$ are defined as

$$
\begin{aligned}
& Q_{1}=Q\left(x_{1}, x_{2}, x_{3}\right), \quad Q_{2}=Q\left(-x_{1}, x_{2}, x_{3}\right), \quad Q_{3}=Q\left(-x_{1},-x_{2}, x_{3}\right), \\
& Q_{4}=Q\left(-x_{1},-x_{2},-x_{3}\right), \quad Q_{5}=Q\left(-x_{1}, x_{2},-x_{3}\right), \quad Q_{6}=Q\left(x_{1},-x_{2}, x_{3}\right), \\
& Q_{7}=Q\left(x_{1},-x_{2},-x_{3}\right), \quad Q_{8}=Q\left(x_{1}, x_{2},-x_{3}\right),
\end{aligned}
$$

and moreover the coefficients $\alpha_{i}, \beta_{i}$ and $\gamma_{i}$ are defined by

$$
\begin{array}{cc}
\alpha_{1}=\alpha_{6}=\alpha_{7}=\alpha_{8}=1-X_{1}, & \alpha_{2}=\alpha_{3}=\alpha_{4}=\alpha_{5}=X_{1}, \\
\beta_{1}=\beta_{2}=\beta_{5}=\beta_{8}=1-X_{2}, & \beta_{3}=\beta_{4}=\beta_{6}=\beta_{7}=X_{2}, \\
\gamma_{1}=\gamma_{2}=\gamma_{3}=\gamma_{6}=1-X_{3}, & \gamma_{4}=\gamma_{5}=\gamma_{7}=\gamma_{8}=X_{3} .
\end{array}
$$

In this way the evaluation of $D_{0}^{\text {cube }}$ is reduced to the one of

$$
D_{i}=\int_{0}^{\alpha_{i}} d x_{1} \int_{0}^{\beta_{i}} d x_{2} \int_{0}^{\gamma_{i}} d x_{3}\left(Q_{i}+B_{4}\right)^{-2-\epsilon / 2}
$$


for which we introduce a Mellin-Barnes transform

$$
D_{i}=\frac{1}{2 \pi i} \int_{-i \infty}^{+i \infty} d s \rho_{4}^{2-s} \mathcal{D}_{i}(s)
$$

where $\rho_{4}=1 / B_{4}$. It follows that

$$
\mathcal{D}_{i}(s)=\alpha_{i} \beta_{i} \gamma_{i} B(s, 2-s) d_{i}(s), \quad d_{i}(s)=\int d C_{3} Q_{i}^{-s}\left(\alpha_{i} x_{1}, \beta_{i} x_{2}, \gamma_{i} x_{3}\right) .
$$

After a sector decomposition in $[0,1]^{3}$ we obtain

$$
d_{i}(s)=\int d C_{3}\left[x_{1}^{2-2 s} Q_{i}^{-s}\left(\alpha_{i}, \beta_{i} x_{2}, \gamma_{i} x_{3}\right)+x_{2}^{2-2 s} Q_{i}^{-s}\left(\alpha_{i} x_{1}, \beta_{i}, \gamma_{i} x_{3}\right)+x_{3}^{2-2 s} Q_{i}^{-s}\left(\alpha_{i} x_{1}, \beta_{i} x_{2}, \gamma_{i}\right)\right] .
$$

Collecting all pieces we obtain

$$
D_{0}^{\text {cube }}=\sum_{i=1}^{8} \sum_{j=1}^{3} \alpha_{i} \beta_{i} \gamma_{i} D_{i j}, \quad D_{i j}=\frac{1}{2 \pi i} \int_{-i \infty}^{+i \infty} d s \rho_{4}^{2-s} \frac{B(s, 2-s)}{3-2 s} \int d C_{2} Q_{i j}^{-s}\left(x_{1}, x_{2}\right) .
$$

The evaluation of $D_{0}^{\text {cube }}$ has been therefore reduced to the one of an inverse Mellin-Barnes transform:

$$
D=\frac{1}{2 \pi i} \int_{-i \infty}^{+i \infty} d s \rho^{2-s} \frac{B(s, 2-s)}{3-2 s} f(s) .
$$

For large values of $|\rho|$ we close the $s$-contour over the right-hand complex half-plane at infinity with a single pole at $s=3 / 2$ and also at $s=2+k, k \geq 0$. The result is straightforward

$$
D=\frac{\pi}{4} f\left(\frac{3}{2}\right) \rho^{1 / 2}-\sum_{k=0}^{\infty} \frac{\Gamma(k+2)}{2 k+1} f(k+2) \frac{(-\rho)^{-k}}{k !} .
$$

Since $f$ stands generically for

$$
f(s) \sim \int d C_{2} Q_{i j}^{-s}\left(x_{1}, x_{2}\right)
$$

we have to evaluate this integral for $s=3 / 2$ as well as for $s$ equal to an arbitrary integer $\geq 2$. We will use the following two recursion relations:

$$
\begin{aligned}
\int d S_{1} Q^{-s}\left(x_{1}\right) & =\frac{1}{2(s-1) B}\left[(2 s-3) \int d S_{1} Q^{1-s}\left(x_{1}\right)+X_{1} Q^{1-s}(0)+\left(1-X_{1}\right) Q^{1-s}(1)\right] \\
\int d C_{2} Q^{-s}\left(x_{1}, x_{2}\right) & =\frac{1}{2(s-1) B}\left\{2(s-2) \int d C_{2} Q^{1-s}\left(x_{1}, x_{2}\right)+\int d S_{1}\left[X_{2} Q_{1}^{1-s}\left(x_{1}\right)\right.\right. \\
& \left.\left.+X_{1} Q_{2}^{1-s}\left(x_{1}\right)+\left(1-X_{2}\right) Q_{3}^{1-s}\left(x_{1}\right)+\left(1-X_{1}\right) Q_{4}^{1-s}\left(x_{1}\right)\right]\right\}
\end{aligned}
$$

where $Q$ is an arbitrary quadratic form in one (two) variables, $B$ is the associated BT factor, $X$ the associated BT co-factor and $Q_{i}$ are secondary quadratic forms defined by

$$
Q_{1}(x)=Q(x, 0), \quad Q_{2}(x)=Q(0, x), \quad Q_{3}(x)=Q(x, 1), \quad Q_{4}(x)=Q(1, x) .
$$


We give in the following the explicit solution for $s=3 / 2$.

$$
\int d C_{2} Q^{-3 / 2}\left(x_{1}, x_{2}\right)=\frac{1}{B}\left[-\frac{3}{B} \int d C_{2} Q^{1 / 2}\left(x_{1}, x_{2}\right)+\int d S_{1} f_{1}\left(\frac{3}{2}\right)-f_{0}\left(\frac{3}{2}\right)\right],
$$

where we have

$$
\begin{aligned}
f_{1}\left(\frac{3}{2}\right) & =X_{2}\left(\frac{2}{B_{1}}+\frac{1}{B}\right) Q_{1}^{1 / 2}\left(x_{1}\right)+X_{1}\left(\frac{2}{B_{2}}+\frac{1}{B}\right) Q_{2}^{1 / 2}\left(x_{1}\right) \\
& +\left(1-X_{2}\right)\left(\frac{2}{B_{3}}+\frac{1}{B}\right) Q_{3}^{1 / 2}\left(x_{1}\right)+\left(1-X_{1}\right)\left(\frac{2}{B_{4}}+\frac{1}{B}\right) Q_{4}^{1 / 2}\left(x_{1}\right), \\
f_{0}\left(\frac{3}{2}\right) & =\frac{X_{2}}{B_{1}}\left[X_{11} Q_{1}^{1 / 2}(0)+\left(1-X_{11}\right) Q_{1}^{1 / 2}(1)\right]+\frac{X_{1}}{B_{2}}\left[X_{12} Q_{2}^{1 / 2}(0)+\left(1-X_{12}\right) Q_{2}^{1 / 2}(1)\right] \\
& +\frac{1-X_{2}}{B_{3}}\left[X_{13} Q_{3}^{1 / 2}(0)+\left(1-X_{13}\right) Q_{3}^{1 / 2}(1)\right] \\
& +\frac{1-X_{1}}{B_{4}}\left[X_{14} Q_{4}^{1 / 2}(0)+\left(1-X_{14}\right) Q_{4}^{1 / 2}(1)\right],
\end{aligned}
$$

where $B$ and $X_{1}, X_{2}$ are the leading BT factor and co-factors of the original quadric while $B_{i}$ and $X_{1 i}$ are the sub-leading ones for the secondary quadratic forms. Note that in this case the BT procedure allows us to derive a result in terms of roots of quadratic forms. For $s=2$ we derive instead

$$
\int d C_{2} Q^{-2}\left(x_{1}, x_{2}\right)=\frac{1}{4 B}\left[\int d S_{1} f_{1}(2)-f_{0}(2)\right]
$$

where we have

$$
\begin{aligned}
f_{1}(2) & =\frac{X_{2}}{B_{1}} \ln Q_{1}\left(x_{1}\right)+\frac{X_{1}}{B_{2}} \ln Q_{2}\left(x_{1}\right)+\frac{1-X_{2}}{B_{3}} \ln Q_{3}\left(x_{1}\right)+\frac{1-X_{1}}{B_{4}} \ln Q_{4}\left(x_{1}\right), \\
f_{0}(2) & =\frac{X_{2}}{B_{1}}\left[X_{11} \ln Q_{1}(0)+\left(1-X_{11}\right) \ln Q_{1}(1)-2\right]+\frac{X_{1}}{B_{2}}\left[X_{12} \ln Q_{2}(0)+\left(1-X_{12}\right) \ln Q_{2}(1)-2\right] \\
& +\frac{1-X_{2}}{B_{3}}\left[X_{13} \ln Q_{3}(0)+\left(1-X_{13}\right) \ln Q_{3}(1)-2\right] \\
& +\frac{1-X_{1}}{B_{4}}\left[X_{14} \ln Q_{4}(0)+\left(1-X_{14}\right) \ln Q_{4}(1)-2\right] .
\end{aligned}
$$

For $s \geq 3$ we derive similar expressions, not to be reported here. $D_{0}^{\text {cube }}$ is, therefore, explicitly known.

The procedure cannot work if one of the sub-leading $B$ is approaching zero. Again, the $B$ are subleading of second type as explained in the discussion before Eq. 85 . In the case $B \approx 0$ the solution consists in a double Mellin-Barnes splitting. Consider Eqs.206)-208), the evaluation of $D_{0}^{\text {cube }}$ is reduced to the one of

$$
D=\frac{1}{2 \pi i} \int_{-i \infty}^{+i \infty} d s \rho_{L}^{2-s} \frac{B(s, 2-s)}{3-2 s} \int d C_{2} Q^{-s}\left(x_{1}, x_{2}\right)
$$

where $Q$ denotes one of the quadratic forms of indices $i j$, which we write as $Q\left(x_{1}, x_{2}\right)=x^{t} H x+2 K^{t} x+L$. Let $B_{S L}=L-K^{t} H^{-1} K, \rho_{S L}=1 / B_{S L}\left(\rho_{L} \equiv \rho_{4}\right)$ and $X=-H^{-1} K$. After transforming $x_{1}, x_{2}$ we obtain

$$
D=\frac{1}{2 \pi i} \int_{-i \infty}^{+i \infty} d s \rho_{L}^{2-s} \frac{B(s, 2-s)}{3-2 s} \int d C_{2}(X)\left(x^{t} H x+B_{S L}\right)^{-s}
$$


With $0<\operatorname{Re} t<\operatorname{Re} s<2$ we write

$$
D=\frac{1}{(2 \pi i)^{2}} \int_{-i \infty}^{+i \infty} d s d t \rho_{L}^{2-s} \rho_{S L}^{s-t} \frac{B(s, 2-s)}{3-2 s} B(t, s-t) \int d C_{2}(X)\left(x^{t} H x\right)^{-t} .
$$

The $x_{1}, x_{2}$ integral has been already considered, starting from Eq.(76), and we know that it develops a pole at $t=1$ times the integral of a quadratic form in one variable. If, for the latter, the BT factor is not around zero we can safely proceed. If we write

$$
\int d C_{2}(X)\left(x^{t} H x\right)^{-t}=\frac{f(t)}{t-1}
$$

then we get

$$
\begin{aligned}
D & =\frac{1}{2 \pi i} \int_{-i \infty}^{+i \infty} d s\left[-f(1) \frac{\rho_{L}^{2}}{\rho_{S L}} \frac{\Gamma(2-s) \Gamma(s-1)}{3-2 s}\left(\frac{\rho_{S L}}{\rho_{L}}\right)^{s}\right. \\
& \left.+\rho_{L}^{2-s} \sum_{k=0}^{\infty} \frac{\Gamma(2-s) \Gamma(s+k-1)}{3-2 s} f(s+k) \frac{\left(-\rho_{S L}\right)^{-k}}{k !}\right] .
\end{aligned}
$$

Next we use $\kappa=\rho_{S L} / \rho_{L}$ and

$$
\begin{aligned}
\frac{1}{2 \pi i} \int_{-i \infty}^{+i \infty} d s \frac{\Gamma(2-s) \Gamma(s-1)}{3-2 s} \kappa^{s} & =\sum_{k=0}^{\infty} \frac{(-1)^{k}}{k !} \frac{\Gamma(k+1)}{2 k+1} \kappa^{1-k} \\
& =\frac{\kappa}{2} \sum_{k=0}^{\infty} \frac{\Gamma(k+1) \Gamma(k+1 / 2)}{\Gamma(k+3 / 2)} \frac{(-\kappa)^{-k}}{k !} \\
& =\kappa_{2} F_{1}\left(\frac{1}{2}, 1 ; \frac{3}{2} ;-\frac{1}{\kappa}\right)=\kappa^{3 / 2} \arctan \left(\kappa^{-1 / 2}\right) .
\end{aligned}
$$

Therefore we get

$$
\begin{aligned}
D & =-\left(\rho_{L} \rho_{S L}\right)^{1 / 2} f(1) \arctan \left(\frac{\rho_{L}}{\rho_{S L}}\right)^{1 / 2}+\frac{1}{2}\left(\pi \rho_{L}\right)^{1 / 2} \sum_{k=0}^{\infty} \Gamma\left(k+\frac{1}{2}\right) f\left(k+\frac{3}{2}\right) \frac{\left(-\rho_{S L}\right)^{-k}}{k !} \\
& -\sum_{k, l=0}^{\infty} \frac{\Gamma(k+l+1) f(k+l+2)}{2 l+1} \frac{\left(-\rho_{S L}\right)^{-k}}{k !} \frac{\left(-\rho_{L}\right)^{-l}}{l !} .
\end{aligned}
$$

In the above results it is always understood that $\rho_{L} \rightarrow \rho_{L}-i \delta$ and that $\rho_{S L} \rightarrow \rho_{S L}-i \delta$.

As illustrated in the previous section the evaluation of $f(k)$ is reduced to computing a one dimensional integral of a quadratic form in one variable (with a small negative imaginary part) which can be done by BT iteration, unless the corresponding BT factor is around zero. If this is the case, we start wondering why the world is against us or return to the starting expression and use one more Mellin-Barnes splitting.

\subsection{A new integral representation for $D_{0}$}

Also for $D_{0}$ we are able to present a new integral representation which is suitable for numerical treatment, especially when $B_{4}=0$. Consider again Eq.(201): after a sector decomposition in $[0,1]^{3}$ we 
obtain

$$
\begin{aligned}
D_{i}= & \alpha_{i} \beta_{i} \gamma_{i} \int d C_{3}\left\{x_{1}^{2}\left[x_{1}^{2} Q_{i}\left(\alpha_{i}, \beta_{i} x_{2}, \gamma_{i} x_{3}\right)+B_{4}-i \delta\right]^{-2-\epsilon / 2}\right. \\
& \left.+x_{2}^{2}\left[x_{2}^{2} Q_{i}\left(\alpha_{i} x_{1}, \beta_{i}, \gamma_{i} x_{3}\right)+B_{4}-i \delta\right]^{-2-\epsilon / 2}+x_{3}^{2}\left[x_{3}^{2} Q_{i}\left(\alpha_{i} x_{1}, \beta_{i} x_{2}, \gamma_{i}\right)+B_{4}-i \delta\right]^{-2-\epsilon / 2}\right\} \\
= & \alpha_{i} \beta_{i} \gamma_{i} \sum_{j=1}^{3} \int d C_{3} x_{3}^{2}\left[x_{3}^{2} Q_{i j}\left(x_{1}, x_{2}\right)+B_{4}-i \delta\right]^{-2-\epsilon / 2}
\end{aligned}
$$

where we introduced the quadratic form

$$
\begin{aligned}
& Q_{i 1}\left(x_{1}, x_{2}\right)=Q_{i}\left(\alpha_{i} x_{1}, \beta_{i} x_{2}, \gamma_{i}\right), \\
& Q_{i 2}\left(x_{1}, x_{2}\right)=Q_{i}\left(\alpha_{i} x_{1}, \beta_{i}, \gamma_{i} x_{2}\right), \\
& Q_{i 3}\left(x_{1}, x_{2}\right)=Q_{i}\left(\alpha_{i}, \beta_{i} x_{1}, \gamma_{i} x_{2}\right) .
\end{aligned}
$$

They are expressible as

$$
Q_{i j}\left(x_{1}, x_{2}\right)=x^{t} H_{i j} x+2 K_{i j}^{t} x+L_{i j}=\bar{x}^{t} M_{i j} \bar{x},
$$

where $x^{t}=\left(x_{1}, x_{2}\right), \bar{x}^{t}=\left(x_{1}, x_{2}, 1\right)$ and the matrix $M$ is given by

$$
M_{i j}=\left(\begin{array}{cc}
H_{i j} & K_{i j} \\
K_{i j}^{t} & L_{i j}
\end{array}\right) .
$$

Now we apply the results for the master integral of Appendix A with coefficients

$$
a=Q_{i j}\left(x_{1}, x_{2}\right), \quad b=B_{4}, \quad \alpha=2+\epsilon / 2, \quad \beta=2 .
$$

With the usual replacement $Q_{i j}, B_{4} \rightarrow Q_{i j}, B_{4}-i \delta$ we obtain

$$
D_{i}=\alpha_{i} \beta_{i} \gamma_{i} \sum_{j=1}^{3} \int d C_{2}\left\{\frac{1}{2} B_{4}^{-1 / 2-\epsilon / 2} B\left(\frac{3}{2}, \frac{1}{2}+\frac{\epsilon}{2}\right) Q_{i j}^{-3 / 2}\left(x_{1}, x_{2}\right)-\int_{0}^{1} d x_{3} x_{3}^{\epsilon} Q_{i j}^{-2-\epsilon / 2}\left(x_{1}, x_{2}, x_{3}\right)\right\} .
$$

In the last equation polynomials $Q_{i j}\left(x_{1}, x_{2}\right)$ and $Q_{i j}\left(x_{1}, x_{2}, x_{3}\right)=Q_{i j}\left(x_{1}, x_{2}\right)+B_{4} x_{3}^{2}$ make their appearance; their powers can be easily incremented with the BT method, as long as the corresponding factors $B_{3 i j}$ are different from zero. The BT relations to be used for this purpose are as follows:

$$
\begin{gathered}
Q_{i j}^{\mu-1}\left(x_{1}, x_{2}\right)=\frac{1}{B_{3 i j}}\left[1-\frac{1}{2 \mu} \sum_{l=1}^{2}\left(x_{l}-X_{l i j}\right) \partial_{x_{l}}\right] Q_{i j}^{\mu}\left(x_{1}, x_{2}\right), \\
Q_{i j}^{\mu-1}\left(x_{1}, x_{2}, x_{3}\right)=\frac{1}{B_{3 i j}}\left[1-\frac{1}{2 \mu}\left(\sum_{l=1}^{2}\left(x_{l}-X_{l i j}\right) \partial_{x_{l}}+x_{3} \partial_{x_{3}}\right)\right] Q_{i j}^{\mu}\left(x_{1}, x_{2}, x_{3}\right),
\end{gathered}
$$

where the sub-leading BT-factors are defined by

$$
B_{3 i j}=\frac{\operatorname{det}\left(M_{i j}\right)}{G_{3 i j}}=\frac{\alpha_{i}^{2} \beta_{i}^{2} \gamma_{i}^{2}}{G_{3 i j}} \operatorname{det}(H), \quad G_{3 i j}=\Delta_{33}\left(M_{i j}\right)
$$




$$
X_{1 i j}=\frac{\Delta_{13}\left(M_{i j}\right)}{G_{3 i j}}, \quad X_{2 i j}=\frac{\Delta_{23}\left(M_{i j}\right)}{G_{3 i j}},
$$

where $\Delta_{l k}\left(M_{i j}\right)$ is the co-determinant of the element $l k$ of the matrix $M_{i j}$. The BT method cannot be used when also some of the $B_{3 i j}$ are near zero and, in this case, we have to proceed along the same lines used for the three-point function. We rewrite the polynomials $Q_{i j}\left(x_{1}, x_{2}\right)$ in the following way:

$$
Q_{i j}\left(x_{1}, x_{2}\right)=\left(x-X_{i j}\right)^{t} H_{i j}\left(x-X_{i j}\right)+B_{3 i j} .
$$

Next we transform variables according to $x_{1}=x_{1}^{\prime}+X_{1 i j}, x_{2}=x_{2}^{\prime}+X_{2 i j}$ and split the $x_{1}-x_{2}$ integration interval according to

$$
\int_{-X_{l i j}}^{1-X_{l i j}} d x_{l}=\left(-\int_{0}^{-X_{l i j}}+\int_{0}^{1-X_{l i j}}\right) d x_{l} .
$$

Each of the integrals is mapped into the unit square and we derive

$$
\begin{aligned}
D_{i} & =\alpha_{i} \beta_{i} \gamma_{i} \sum_{j=1}^{3} \sum_{l=1}^{4} \eta_{i j l} \xi_{i j l} \int d C_{2}\left\{\frac{1}{2} B_{4}^{-1 / 2-\epsilon / 2} B\left(\frac{3}{2}, \frac{1}{2}+\frac{\epsilon}{2}\right)\left[Q_{i j l}\left(x_{1}, x_{2}\right)+B_{3 i j}\right]^{-3 / 2}\right. \\
& \left.-\int_{0}^{1} d x_{3} x_{3}^{\epsilon}\left[Q_{i j l}\left(x_{1}, x_{2}\right)+B_{3 i j}+B_{4} x_{3}^{2}\right]^{-2-\epsilon / 2}\right\},
\end{aligned}
$$

where the replacement $B_{3 i j} \rightarrow B_{3 i j}-i \delta$ is understood and where we introduced new auxiliary quantities:

$$
\begin{array}{ll}
\eta_{i j 1}=\eta_{i j 2}=1-X_{1 i j}, & \eta_{i j 3}=\eta_{i j 4}=X_{1 i j}, \\
\xi_{i j 1}=\eta_{i j 3}=1-X_{2 i j}, & \xi_{i j 2}=\xi_{i j 4}=X_{2 i j} .
\end{array}
$$

The new quadrics are defined by

$$
\begin{aligned}
& Q_{(i j) 1}=Q_{i j}^{\prime}\left(\left(1-X_{1 i j}\right) x_{1},\left(1-X_{2 i j}\right) x_{2}\right), \quad Q_{(i j) 2}=Q_{i j}^{\prime}\left(\left(1-X_{1 i j}\right) x_{1},-X_{2 i j} x_{2}\right), \\
& Q_{(i j) 3}=Q_{i j}^{\prime}\left(-X_{1 i j} x_{1},\left(1-X_{2 i j}\right) x_{2}\right), \quad Q_{(i j) 4}=Q_{i j}^{\prime}\left(-X_{1 i j} x_{1},-X_{2 i j} x_{2}\right),
\end{aligned}
$$

with $Q_{i j}^{\prime}=x^{t} H_{i j} x$. At this point, after performing the usual sector decomposition of the square $[0,1]^{2}$, we apply again the master formula of Appendix A and get:

$$
\begin{gathered}
D_{i}=\alpha_{i} \beta_{i} \gamma_{i} \sum_{j=1}^{3} \sum_{l=1}^{4} \sum_{m=1}^{2} \eta_{i j l} \xi_{i j l} \int_{0}^{1} d x_{1} D_{i j l m} \\
D_{i j l m}=\frac{1}{2} B_{4}^{-1 / 2-\epsilon / 2} B_{3 i j}^{-1 / 2} B\left(\frac{3}{2}, \frac{1}{2}+\frac{\epsilon}{2}\right) Q_{i j l m}^{-1}\left(x_{1}\right) \\
-\frac{1}{2} B(1,1+\epsilon / 2) Q_{i j l m}^{-1}\left(x_{1}\right) \int_{0}^{1} d x_{3} x_{3}^{\epsilon}\left[B_{4} x_{3}^{2}+B_{3 i j}\right]^{-1-\epsilon / 2} \\
-\frac{1}{2} B_{4}^{-1 / 2-\epsilon / 2} B\left(\frac{3}{2}, \frac{1}{2}+\frac{\epsilon}{2}\right) \int_{0}^{1} d x_{2} Q_{i j l m}^{-3 / 2}\left(x_{1}, x_{2}\right)+\int_{0}^{1} d x_{2} \int_{0}^{x_{2}} d x_{3} x_{3}^{\epsilon} Q_{i j l m}^{-2-\epsilon / 2}\left(x_{1}, x_{2}, x_{3}\right),
\end{gathered}
$$

where we introduced $Q_{i j l 1}\left(x_{1}\right)=Q_{i j l}\left(x_{1}, 1\right)$ and $Q_{i j l 2}\left(x_{1}\right)=Q_{i j l}\left(1, x_{1}\right)$. Likewise we have

$$
\begin{aligned}
Q_{i j l m}\left(x_{1}, x_{2}\right) & =Q_{i j l m}\left(x_{1}\right)+B_{3 i j} x_{2}^{2}, \\
Q_{i j l m}\left(x_{1}, x_{2}, x_{3}\right) & =Q_{i j l m}\left(x_{1}\right)+B_{3 i j} x_{2}^{2}+B_{4} x_{3}^{2} .
\end{aligned}
$$


The divergent behavior of the first and of the third term is evident and the second integral depends essentially on the ratio

$$
\kappa_{i j}=\frac{B_{4}-i \delta}{B_{3 i j}-i \delta}
$$

Therefore, by applying the master formula of Appendix A, we obtain

$$
\begin{aligned}
\int_{0}^{1} d x_{3} x_{3}^{\epsilon}\left[B_{4} x_{3}^{2}+B_{3 i j}\right]^{-1-\epsilon / 2} & =\frac{1}{2} B_{3 i j}^{-1 / 2} B_{4}^{-1 / 2-\epsilon / 2} B\left(\frac{1}{2}+\frac{\epsilon}{2}, \frac{1}{2}\right)-\int_{0}^{1} d x_{3}\left[B_{3 i j} x_{3}^{2}+B_{4}\right]^{-1-\epsilon / 2} \\
& =\frac{\pi}{2} B_{3 i j}^{-1 / 2} B_{4}^{-1 / 2}-B_{4}^{-1} \kappa_{i j}^{1 / 2} \arctan \left(\kappa_{i j}^{-1 / 2}\right)+\mathcal{O}(\epsilon)
\end{aligned}
$$

Note that the first term in Eq.(241) cancels the first term of Eq.(238) giving our integral representation for $D_{i}$ :

$$
\begin{aligned}
D_{i} & =\alpha_{i} \beta_{i} \gamma_{i} \sum_{j=1}^{3} \sum_{l=1}^{4} \sum_{m=1}^{2} \eta_{i j l} \xi_{i j l} \int_{0}^{1} d x_{1}\left\{\frac{1}{2} B_{4}^{-1 / 2} B_{3 i j}^{-1 / 2} \arctan \left(\kappa_{i j}^{-1 / 2}\right) Q_{i j l m}^{-1}\left(x_{1}\right)\right. \\
& \left.-\frac{\pi}{4} B_{4}^{-1 / 2} \int_{0}^{1} d x_{2} Q_{i j l m}^{-3 / 2}\left(x_{1}, x_{2}\right)+\int_{0}^{1} d x_{2} \int_{0}^{x_{2}} d x_{3} Q_{i j l m}^{-2}\left(x_{1}, x_{2}, x_{3}\right)\right\} .
\end{aligned}
$$

As a final step, the power of the polynomials appearing in Eq.(242) can be incremented by using the following set of BT relations:

$$
\begin{gathered}
Q_{i j l m}^{-1}\left(x_{1}\right)=\frac{1}{b_{i j l m}}\left[1-\frac{1}{2}\left(x_{1}+\frac{k_{i j l m}}{h_{i j l m}}\right) \partial_{x_{1}} \ln Q_{i j l m}\left(x_{1}\right)\right], \\
Q_{i j l m}^{\mu-1}\left(x_{1}, x_{2}\right)=\frac{1}{b_{i j l m}}\left\{1-\frac{1}{2 \mu}\left[\left(x_{1}+\frac{k_{i j l m}}{h_{i j l m}}\right) \partial_{x_{1}}+x_{2} \partial_{x_{2}}\right]\right\} Q_{i j l m}^{\mu}\left(x_{1}, x_{2}\right), \\
Q_{i j l m}^{\mu-1}\left(x_{1}, x_{2}, x_{3}\right)=\frac{1}{b_{i j l m}}\left\{1-\frac{1}{2 \mu}\left[\left(x_{1}+\frac{k_{i j l m}}{h_{i j l m}}\right) \partial_{x_{1}}+\sum_{k=2}^{3} x_{k} \partial_{x_{k}}\right]\right\} Q_{i j l m}^{\mu}\left(x_{1}, x_{2}, x_{3}\right),
\end{gathered}
$$

where we defined

$$
Q_{i j l m}\left(x_{1}\right)=h_{i j l m} x_{1}^{2}+2 k_{i j l m} x_{1}+l_{i j l m} \quad b_{i j l m}=l_{i j l m}-\frac{k_{i j l m}^{2}}{h_{i j l m}} .
$$

If also $b_{i j l m}$ tends to 0 , we must repeat the procedure for the quadratic form $Q_{i j l m}\left(x_{1}\right)$.

\section{$7 \quad$ Infrared divergent $D_{0}$}

At the one-loop level the evaluation of infrared divergent $N$-point functions with $N \geq 4$ is greatly simplified insofar the divergent parts can always be reduced to three-point functions. This decomposition is even more relevant in our approach where the evaluation of the infrared finite remainder does not 
require any additional work. Let us give an example for $D_{0}$ [21], where we consider the configuration with

$$
p_{1}^{2}=p_{2}^{2}=-m_{2}^{2}, \quad p_{3}^{2}=p_{4}^{2}=-m_{4}^{2}, \quad m_{1}=m_{3}=0 .
$$

If we introduce the following notation:

$$
[1]=q^{2}, \quad[2]=\left(q+p_{1}\right)^{2}+m_{2}^{2}, \quad[3]=(q+P)^{2}, \quad[4]=\left(q-p_{4}\right)^{2}+m_{4}^{2},
$$

where $P=p_{1}+p_{2}$, then the following decomposition holds

$$
\begin{aligned}
-P^{2} D_{0} & =\frac{2}{i \pi^{2}} \int d^{d} q \frac{q \cdot(q+P)}{[1][2][3][4]}-C_{0}\left(\left(p_{2}+p_{3}\right)^{2}, p_{2}^{2}, p_{3}^{2} ; m_{2}, 0, m_{4}\right) \\
& -C_{0}\left(p_{4}^{2}, p_{1}^{2},\left(p_{2}+p_{3}\right)^{2} ; 0, m_{2}, m_{4}\right),
\end{aligned}
$$

showing two IR divergent $C_{0}$ functions and an IR finite integral. For the latter we obtain

$$
D_{0}^{\mathrm{rem}}=\frac{1}{i \pi^{2}} \int d^{d} q \frac{q \cdot(q+P)}{[1][2][3][4]}=\int d S_{3} V^{-2-\epsilon / 2}\left(k^{2}-k \cdot P+2 V\right),
$$

where

$$
P=p_{1}+p_{2}, \quad Q=p_{2}+p_{3}, \quad k=\sum_{i=1}^{3} x_{i} p_{i}
$$

and the quadric

$$
\begin{aligned}
V & =m_{2}^{2}\left(x_{1}^{2}+x_{2}^{2}\right)+m_{4}^{2} x_{3}^{2}-\left(P^{2}+2 m_{2}^{2}\right) x_{1} x_{2}+\left(Q^{2}+P^{2}+m_{2}^{2}+m_{4}^{2}\right) x_{1} x_{3} \\
& -\left(Q^{2}+m_{2}^{2}+m_{4}^{2}\right) x_{2} x_{3}+P^{2}\left(x_{2}-x_{3}\right),
\end{aligned}
$$

shows a zero for $x_{1}=x_{2}=x_{3}=0$. The numerator in Eq.(250) becomes

$$
k^{2}-k \cdot P+2 V=V-\frac{1}{2} P^{2}\left(x_{1}-x_{2}+x_{3}\right),
$$

so that we obtain

$$
D_{0}^{\mathrm{rem}}=\int d S_{3}\left[V^{-1-\epsilon / 2}-\frac{1}{2} P^{2}\left(x_{1}-x_{2}+x_{3}\right) V^{-2-\epsilon / 2}\right] .
$$

By infrared power counting the expression for $D_{0}^{\text {rem }}$ is manifestly finite and can be computed according to standard BT techniques when $B_{4} \neq 0$. The explicit expression of the coefficient $B_{4}$ is

$$
B_{4}=\frac{1}{\operatorname{det}(H)} \frac{P^{4}}{16}\left[4 m_{2}^{2} m_{4}^{2}-\left(Q^{2}+m_{2}^{2}+m_{4}^{2}\right)^{2}\right]=\frac{1}{\operatorname{det}(H)} \frac{P^{4}}{16} \lambda\left(-Q^{2}, m_{2}^{2}, m_{4}^{2}\right),
$$

where $\operatorname{det}(H)$ is the determinant of the matrix $H$ in the BT relation:

$$
\operatorname{det}(H)=\frac{P^{2}}{4}\left[\lambda\left(-Q^{2}, m_{2}^{2}, m_{4}^{2}\right)+Q^{2} P^{2}\right] .
$$

As a consequence $B_{4}=0$ only for $Q^{2}<0$, i.e. for normal(pseudo)-threshold $Q^{2}=-\left(m_{2} \pm m_{4}\right)^{2}$. Around the thresholds other techniques are used since the BT procedure overestimates the singularity, see Section 6.4 for the pseudo-threshold (where $D_{0}^{\text {rem }}$ is regular) and Section 6.5 for normal threshold (where $D_{0}^{\text {rem }}$ is indeed singular). 


\subsection{Classification of IR divergent cases for the D-family}

A short classification of IR divergent four-point functions can be based on IR power counting which, for one-loop diagrams, requires $\int_{0} d^{4} q / q^{4}$. The configurations of interest are those where some of the internal masses vanish and the Mandelstam invariants of the process are not constrained.

Without loss of generality we may assume that $m_{1}=0$. For $q=\Delta$ and $\Delta$ vanishing the requested behavior of $\mathcal{O}\left(\Delta^{4}\right)$ is obtained in three different cases ?:

1. $p_{1}^{2}=-m_{2}^{2} \quad p_{4}^{2}=-m_{4}^{2}$;

2. $p_{1}^{2}=-m_{2}^{2} \quad P^{2}=-m_{3}^{2}$;

3. $p_{4}^{2}=-m_{4}^{2} \quad P^{2}=-m_{3}^{2}$.

Note that only the first one is of interest, so the IR configuration is

$$
m_{1}=0, \quad p_{1}^{2}=-m_{2}^{2}, \quad p_{4}^{2}=-m_{4}^{2} .
$$

If also $m_{3}=0$ then there is an additional pole for $q=-P$ and we introduce $q=-P+\Delta$. The four denominators in $D_{0}$ behave as

$$
[1] \sim P^{2}, \quad[2] \sim p_{2}^{2}+m_{2}^{2}-2 p_{2} \cdot \Delta, \quad[3] \sim \Delta^{2}, \quad[4] \sim p_{3}^{2}+m_{4}^{2}+2 p_{3} \cdot \Delta,
$$

and the requested behavior of $\mathcal{O}\left(\Delta^{4}\right)$ is obtained if $p_{2}^{2}=-m_{2}^{2}$ and $p_{3}^{2}=-m_{4}^{2}$, as expected. Therefore another IR configuration is

$$
m_{1}=m_{3}=0, \quad\left\{p_{1}^{2}=-m_{2}^{2} p_{4}^{2}=-m_{4}^{2}\right\} \quad \text { and } / \text { or } \quad\left\{p_{2}^{2}=-m_{2}^{2} p_{3}^{2}=-m_{4}^{2}\right\} .
$$

If instead we have $m_{1}=0$ and $m_{2}=0$ there will be another pole at $q=-p_{1}$ whose IR nature depends on $p_{1}^{2}=0$ and $p_{2}^{2}=-m_{3}^{2}$. The new IR configuration is

$$
m_{1}=m_{2}=0, p_{1}^{2}=0, \quad\left\{p_{4}^{2}=-m_{4}^{2}\right\} \quad \text { and } / \text { or } \quad\left\{p_{2}^{2}=-m_{3}^{2}\right\} .
$$

Furthermore, if $m_{1}=m_{2}=m_{3}=0$ there are the following IR configurations:

$$
m_{1}=m_{2}=m_{3}=0, \quad\left\{p_{1}^{2}=0 p_{4}^{2}=-m_{4}^{2}\right\} \quad \text { or } \quad\left\{p_{1}^{2}=0 p_{2}^{2}=0\right\} \quad \text { or } \quad\left\{p_{2}^{2}=0 p_{3}^{2}=-m_{4}^{2}\right\} .
$$

In all cases the IR box can be reduced to a combination of (possibly) IR $C_{0}$ functions and a IR finite reminder.

To further clarify the connection between IR configurations and Landau singularities we consider the $D_{0}$ specified by Eq.(247). The corresponding Landau equations are

$$
\begin{aligned}
& \alpha_{1} q^{2}=0, \quad \alpha_{2}\left[\left(q+p_{1}\right)^{2}+m_{2}^{2}\right]=0, \quad \alpha_{3}(q+P)^{2}=0, \quad \alpha_{4}\left[\left(q-p_{4}\right)^{2}+m_{4}^{2}\right]=0 \\
& \alpha_{1} q_{\mu}+\alpha_{2}\left(q+p_{1}\right)_{\mu}+\alpha_{3}(q+P)_{\mu}+\alpha_{4}\left(q-p_{4}\right)_{\mu}=0 .
\end{aligned}
$$

The equations in the first row of Eq.(262) are satisfied, for $\alpha_{i} \neq 0$, if

$$
q^{2}=0, \quad q \cdot p_{1}=0, \quad q \cdot P=-\frac{1}{2} P^{2}, \quad q \cdot p_{4}=0 .
$$

\footnotetext{
${ }^{5}$ This is the well-known eikonal approximation [22], whose validity we assume without further justifications [23].
} 
By contracting the free Lorentz index in the equation in the second row of Eq.(262) by $q_{\mu},\left(p_{1}\right)_{\mu}, P_{\mu}$, and $p_{4 \mu}$, and by using Eq.(263) we obtain the system of equations

$$
\begin{aligned}
\alpha_{3} P^{2}=0, & -2 \alpha_{2} m_{2}^{2}+\alpha_{3} P^{2}-2 \alpha_{4} p_{1} \cdot p_{4}=0, \\
-\alpha_{1} P^{2}+\alpha_{3} P^{2} & =0, \quad 2 \alpha_{2} p_{1} \cdot p_{4}-\alpha_{3} P^{2}+2 \alpha_{4} m_{4}^{2}=0 .
\end{aligned}
$$

If the system of Eq.(264) admits a proper solution (i.e. a solution in which not all the $\alpha_{i}$ vanish), the determinant of the system must be equal to zero. One can readily verify that the determinant is

$$
\Delta=-\frac{P^{4}}{16} \lambda\left(-Q^{2}, m_{2}^{2}, m_{4}^{2}\right)
$$

Therefore the leading Landau singularity requires $Q^{2}<0$ and corresponds to the usual normal threshold $Q^{2}=-\left(m_{2}+m_{4}\right)^{2}$ on top of the infrared pole.

\section{Five-point functions (E-family)}

Several results are known in the literature about the reduction of dimensionally regulated one-loop $N$-point integrals with $N \geq 5$ ([5], [24] and [25]). The generic, scalar, pentagon is given by

$$
E_{0}=\int d S_{4} V^{-3-\epsilon / 2}\left(x_{1}, x_{2}, x_{3}, x_{4}\right), \quad V(x)=x^{t} H x+2 K^{t} x+L,
$$

where $H_{i j}=-p_{i} \cdot p_{j}$ with $i, j=1, \ldots, 4, L=m_{1}^{2}$, and where

$$
\begin{aligned}
& K_{1}=-\frac{1}{2}\left(m_{1}^{2}-m_{2}^{2}-p_{1} \cdot p_{1}\right) \\
& K_{2}=-\frac{1}{2}\left(m_{2}^{2}-m_{3}^{2}-2 p_{1} \cdot p_{2}-p_{2} \cdot p_{2}\right) \\
& K_{3}=-\frac{1}{2}\left(m_{3}^{2}-m_{4}^{2}-2 p_{1} \cdot p_{3}-2 p_{2} \cdot p_{3}-p_{3} \cdot p_{3}\right) \\
& K_{4}=-\frac{1}{2}\left(m_{4}^{2}-m_{5}^{2}-2 p_{1} \cdot p_{4}-2 p_{2} \cdot p_{4}-2 p_{3} \cdot p_{4}-p_{4} \cdot p_{4}\right)
\end{aligned}
$$

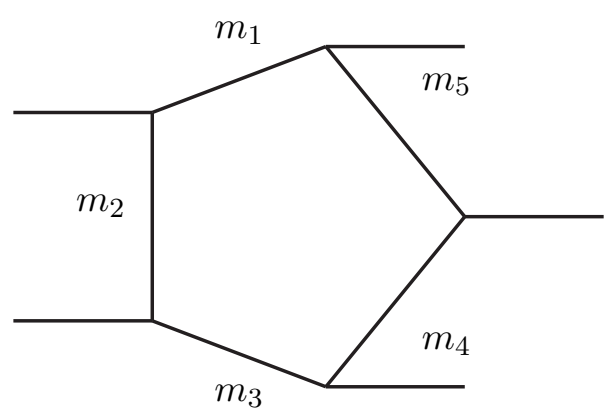

Figure 4: The one-loop, five-point Green function of Eq.(266). Propagators are $q^{2}+m_{1}^{2} \cdots\left(q+p_{1}+\cdots+p_{4}\right)^{2}+m_{5}^{2}$. 


\subsection{Evaluation of $E_{0}$ when $B_{5} \neq 0$}

It is a virtue of the $\mathrm{BT}$ algorithm that we can show the decomposition of $E_{0}$ in five boxes (in $d=4$ ) with just one iteration, as depicted in Fig. 0 . We obtain

$$
E_{0}=\frac{1}{4 B_{5}} \sum_{i=0}^{4} w_{i} D_{0}^{(i)},
$$

where the weights are

$$
w_{i}=X_{i}-X_{i+1}, \quad X_{0}=1, \quad X_{5}=0,
$$

and where $B_{5}=L-K^{t} H^{-1} K$ and $X=-K^{t} H^{-1}$. The further advantage in this derivation is that the nature of the weights is transparent since $B_{5}=0$ corresponds to a Landau singularity of the pentagon. Furthermore the boxes are specified by

$$
D_{0}^{(i)}=\int d S_{3} V^{-2-\epsilon / 2}(\widehat{i i+1})
$$

where the contractions are

$$
\widehat{01}=\left(1, x_{1}, x_{2}, x_{3}\right), \quad \widehat{45}=\left(x_{1}, x_{2}, x_{3}, 0\right),
$$

etc. As long as $B_{5}$ is not around zero the derivation for the pentagon is completed since we know how to deal with boxes even if their (sub-leading) BT factors are around zero.

\subsection{Form factors in the E-family}

The nice feature of the scalar pentagon is that fourfold integrals disappear in the final answer, Eq.(268). The same is not immediately true for form factors, $E_{11}$ etc, which contain powers of the Feynman parameters in the numerator. We can use a new identity,

$$
\left(x_{i}-X_{i}\right) V^{\mu}\left(x_{1}, x_{2}, x_{3}, x_{4}\right)=\frac{1}{2(\mu+1)} H_{i j}^{-1} \partial_{j} V^{\mu+1}\left(x_{1}, x_{2}, x_{3}, x_{4}\right) .
$$

With the help of the identity and after integration-by-parts we are able to remove again all fourfold integrals in the form factors of the $E_{1 i}$-series. To give an example we consider

$$
E_{11}=\int d S_{4} x_{1} V^{-3-\epsilon / 2}\left(x_{1}, x_{2}, x_{3}, x_{4}\right)=\frac{1}{4 B_{5}} \int d S_{3}\left(E_{11}^{-2}-\frac{1}{2} E_{11}^{-1}\right) .
$$

Secondary quadrics are defined as:

$$
\begin{array}{ll}
V_{a}\left(x_{1}, x_{2}, x_{3}\right)=V\left(x_{1}, x_{1}, x_{2}, x_{3}\right), & V_{b}\left(x_{1}, x_{2}, x_{3}\right)=V\left(x_{1}, x_{2}, x_{2}, x_{3}\right), \\
V_{c}\left(x_{1}, x_{2}, x_{3}\right)=V\left(x_{1}, x_{2}, x_{3}, x_{3}\right), & V_{d}\left(x_{1}, x_{2}, x_{3}\right)=V\left(x_{1}, x_{2}, x_{3}, 0\right), \\
V_{e}\left(x_{1}, x_{2}, x_{3}\right)=V\left(1, x_{1}, x_{2}, x_{3}\right) . &
\end{array}
$$

The $E_{11}$ form factor is fully specified by

$$
\begin{aligned}
E_{11}^{-2} & =\left(X_{1}-X_{2}\right) x_{1} V_{a}^{-2-\epsilon / 2}+\left(X_{2}-X_{3}\right) x_{1} V_{b}^{-2-\epsilon / 2}+\left(X_{3}-X_{4}\right) x_{1} V_{c}^{-2-\epsilon / 2} \\
& +X_{4} x_{1} V_{d}^{-2-\epsilon / 2}+\left(1-X_{1}\right) V_{e}^{-2-\epsilon / 2}
\end{aligned}
$$




$$
\begin{aligned}
E_{11}^{-1} & =\left(H_{11}^{-1}-H_{12}^{-1}\right) V_{a}^{-1-\epsilon / 2}+\left(H_{12}^{-1}-H_{13}^{-1}\right) V_{b}^{-1-\epsilon / 2}+\left(H_{13}^{-1}-H_{14}^{-1}\right) V_{c}^{-1-\epsilon / 2} \\
& +H_{14}^{-1} V_{d}^{-1-\epsilon / 2}-H_{11}^{-1} V_{e}^{-1-\epsilon / 2} .
\end{aligned}
$$

Similarly we obtain

$$
\begin{aligned}
E_{i 1}^{-1} & =\left(H_{i 1}^{-1}-H_{i 2}^{-1}\right) V_{a}^{-1-\epsilon / 2}+\left(H_{i 2}^{-1}-H_{i 3}^{-1}\right) V_{b}^{-1-\epsilon / 2}+\left(H_{i 3}^{-1}-H_{i 4}^{-1}\right) V_{c}^{-1-\epsilon / 2} \\
& +H_{i 4}^{-1} V_{d}^{-1-\epsilon / 2}-H_{i 1}^{-1} V_{e}^{-1-\epsilon / 2}
\end{aligned}
$$

for $i=1, \ldots, 4$ and

$$
\begin{aligned}
E_{21}^{-2} & =\left(X_{1}-X_{2}\right) x_{1} V_{a}^{-2-\epsilon / 2}+\left(X_{2}-X_{3}\right) x_{2} V_{b}^{-2-\epsilon / 2}+\left(X_{3}-X_{4}\right) x_{2} V_{c}^{-2-\epsilon / 2} \\
& +X_{4} x_{2} V_{d}^{-2-\epsilon / 2}+\left(1-X_{1}\right) x_{1} V_{e}^{-2-\epsilon / 2}, \\
E_{31}^{-2} & =\left(X_{1}-X_{2}\right) x_{2} V_{a}^{-2-\epsilon / 2}+\left(X_{2}-X_{3}\right) x_{2} V_{b}^{-2-\epsilon / 2}+\left(X_{3}-X_{4}\right) x_{3} V_{c}^{-2-\epsilon / 2} \\
& +X_{4} x_{3} V_{d}^{-2-\epsilon / 2}+\left(1-X_{1}\right) x_{2} V_{e}^{-2-\epsilon / 2}, \\
E_{41}^{-2} & =\left(X_{1}-X_{2}\right) x_{3} V_{a}^{-2-\epsilon / 2}+\left(X_{2}-X_{3}\right) x_{3} V_{b}^{-2-\epsilon / 2}+\left(X_{3}-X_{4}\right) x_{3} V_{c}^{-2-\epsilon / 2} \\
& +\left(1-X_{1}\right) x_{3} V_{e}^{-2-\epsilon / 2} .
\end{aligned}
$$

As it has been done before and for the reader's convenience we list all the form factors in the $E$-family. Note that there are also delicate points connected with the decomposition into objects belonging to the $E$-family since the decomposition itself is strictly defined in 4 dimensions. Furthermore, starting from six powers of momenta in the numerator, we will encounter UV divergent terms so that some care is needed. Clearly we can have such a term while working in a general $R_{\xi}$-gauge.

With two momenta in the numerator we define form factors as

$$
\begin{aligned}
& E_{2 ; i}=E_{2}(i i), \quad i, j=1, \cdots, 4, \\
& E_{2 ; 5}=E_{2}(12), \quad E_{2 ; 6}=E_{2}(13), \quad E_{2 ; 7}=E_{2}(14), \quad E_{2 ; 8}=E_{2}(23), \\
& E_{2 ; 9}=E_{2}(24), \quad E_{2 ; 10}=E_{2}(34),
\end{aligned}
$$

where the auxiliary function $E_{2}(i j)$ is

$$
E_{2}(i j)=\int d S_{4} x_{i} x_{j} V^{-3-\epsilon / 2}\left(x_{1}, x_{2}, x_{3}, x_{4}\right) .
$$

However, a new form factor arises, the one proportional to $\delta_{\mu \nu}$,

$$
E_{2 ; 11}=\frac{1}{4} \int d S_{4} V^{-2-\epsilon / 2}\left(x_{1}, x_{2}, x_{3}, x_{4}\right) .
$$

$E_{2 ; 11}$ can still be reduced to form factors of the $E$-family by writing

$$
E_{2 ; 11}=\frac{1}{4}\left[\sum_{i, j=1}^{4} H_{i j} E_{2}(i j)+2 \sum_{i=1}^{4} K_{i} E_{1 i}+L E_{0}\right] .
$$

Let us give the complete expressions for the form factors of the $E_{2}$-series.

$$
4 B_{5} E_{2}(i j)=-\int d S_{4} H_{i j}^{-1} V^{-1-\epsilon / 2}\left(x_{1}, x_{2}, x_{3}, x_{4}\right)+\int d S_{3}\left(E_{2 ; i j}^{-2}-E_{2 ; i j}^{-1}\right),
$$


where the various coefficients are:

$$
\begin{aligned}
& E_{2 ; 11}^{-2}=\left(X_{1}-X_{2}\right) x_{1}^{2} V_{a}^{-2-\epsilon / 2}+\left(X_{2}-X_{3}\right) x_{1}^{2} V_{b}^{-2-\epsilon / 2} \\
& +\left(X_{3}-X_{4}\right) x_{1}^{2} V_{c}^{-2-\epsilon / 2}+X_{4} x_{1}^{2} V_{d}^{-2-\epsilon / 2}+\left(1-X_{1}\right) V_{e}^{-2-\epsilon / 2}, \\
& E_{2 ; 22}^{-2}=\left(X_{1}-X_{2}\right) x_{1}^{2} V_{a}^{-2-\epsilon / 2}+\left(X_{2}-X_{3}\right) x_{2}^{2} V_{b}^{-2-\epsilon / 2} \\
& +\left(X_{3}-X_{4}\right) x_{2}^{2} V_{c}^{-2-\epsilon / 2}+X_{4} x_{2}^{2} V_{d}^{-2-\epsilon / 2}+\left(1-X_{1}\right) x_{1}^{2} V_{e}^{-2-\epsilon / 2}, \\
& E_{2 ; 33}^{-2}=\left(X_{1}-X_{2}\right) x_{2}^{2} V_{a}^{-2-\epsilon / 2}+\left(X_{2}-X_{3}\right) x_{2}^{2} V_{b}^{-2-\epsilon / 2} \\
& +\left(X_{3}-X_{4}\right) x_{3}^{2} V_{c}^{-2-\epsilon / 2}+X_{4} x_{3}^{2} V_{d}^{-2-\epsilon / 2}+\left(1-X_{1}\right) x_{2}^{2} V_{e}^{-2-\epsilon / 2}, \\
& E_{2 ; 44}^{-2}=\left(X_{1}-X_{2}\right) x_{3}^{2} V_{a}^{-2-\epsilon / 2}+\left(X_{2}-X_{3}\right) x_{3}^{2} V_{b}^{-2-\epsilon / 2} \\
& +\left(X_{3}-X_{4}\right) x_{3}^{2} V_{c}^{-2-\epsilon / 2}+\left(1-X_{1}\right) x_{3}^{2} V_{e}^{-2-\epsilon / 2}, \\
& E_{2 ; 12}^{-2}=\left(X_{1}-X_{2}\right) x_{1}^{2} V_{a}^{-2-\epsilon / 2}+\left(X_{2}-X_{3}\right) x_{1} x_{2} V_{b}^{-2-\epsilon / 2} \\
& +\left(X_{3}-X_{4}\right) x_{1} x_{2} V_{c}^{-2-\epsilon / 2}+X_{4} x_{1} x_{2} V_{d}^{-2-\epsilon / 2}+\left(1-X_{1}\right) x_{1} V_{e}^{-2-\epsilon / 2}, \\
& E_{2 ; 11}^{-1}=\left(H_{11}^{-1}-H_{12}^{-1}\right) x_{1} V_{a}^{-1-\epsilon / 2}+\left(H_{12}^{-1}-H_{13}^{-1}\right) x_{1} V_{b}^{-1-\epsilon / 2} \\
& +\left(H_{13}^{-1}-H_{14}^{-1}\right) x_{1} V_{c}^{-1-\epsilon / 2}+H_{14}^{-1} x_{1} V_{d}^{-1-\epsilon / 2}-H_{11}^{-1} V_{e}^{-1-\epsilon / 2}, \\
& E_{2 ; 22}^{-1}=\left(H_{21}^{-1}-H_{22}^{-1}\right) x_{1} V_{a}^{-1-\epsilon / 2}+\left(H_{22}^{-1}-H_{23}^{-1}\right) x_{2} V_{b}^{-1-\epsilon / 2} \\
& +\left(H_{23}^{-1}-H_{24}^{-1}\right) x_{2} V_{c}^{-1-\epsilon / 2}+H_{24}^{-1} x_{2} V_{d}^{-1-\epsilon / 2}-H_{21}^{-1} x_{1} V_{e}^{-1-\epsilon / 2}, \\
& E_{2 ; 33}^{-1}=\left(H_{31}^{-1}-H_{32}^{-1}\right) x_{2} V_{a}^{-1-\epsilon / 2}+\left(H_{32}^{-1}-H_{33}^{-1}\right) x_{2} V_{b}^{-1-\epsilon / 2} \\
& +\left(H_{33}^{-1}-H_{34}^{-1}\right) x_{3} V_{c}^{-1-\epsilon / 2}+H_{34}^{-1} x_{3} V_{d}^{-1-\epsilon / 2}-H_{31}^{-1} x_{2} V_{e}^{-1-\epsilon / 2}, \\
& E_{2 ; 44}^{-1}=\left(H_{41}^{-1}-H_{42}^{-1}\right) x_{3} V_{a}^{-1-\epsilon / 2}+\left(H_{42}^{-1}-H_{43}^{-1}\right) x_{3} V_{b}^{-1-\epsilon / 2} \\
& +\left(H_{43}^{-1}-H_{44}^{-1}\right) x_{3} V_{c}^{-1-\epsilon / 2}-H_{41}^{-1} x_{3} V_{e}^{-1-\epsilon / 2}, \\
& 2 E_{2 ; 12}^{-1}=\left(H_{11}^{-1}-H_{22}^{-1}\right) x_{1} V_{a}^{-1-\epsilon / 2}+\left[\left(H_{12}^{-1}-H_{13}^{-1}\right) x_{2}+\left(H_{22}^{-1}-H_{23}^{-1}\right) x_{1}\right] V_{b}^{-1-\epsilon / 2} \\
& +\left[\left(H_{13}^{-1}-H_{14}^{-1}\right) x_{2}+\left(H_{23}^{-1}-H_{24}^{-1}\right) x_{1}\right] V_{c}^{-1-\epsilon / 2} \\
& +\left(H_{14}^{-1} x_{2}+H_{24}^{-1} x_{1}\right) V_{d}^{-1-\epsilon / 2}-\left(H_{11}^{-1} x_{1}+H_{21}^{-1}\right) V_{e}^{-1-\epsilon / 2} .
\end{aligned}
$$

All functions $E_{i j}$ contain a term which is proportional to $E_{0}(d=8)$. Consider again $E_{2 ; 11}$ which becomes

$$
\begin{aligned}
4 B_{5} E_{2 ; 11} & =-E_{0}(d=8)+\frac{1}{2} \int d S_{3}\left[\left(X_{1}-X_{2}\right) V_{a}^{-1-\epsilon / 2}+\left(X_{2}-X_{3}\right) V_{b}^{-1-\epsilon / 2}\right. \\
& \left.+\left(X_{3}-X_{4}\right) V_{c}^{-1-\epsilon / 2}+X_{4} V_{d}^{-1-\epsilon / 2}+\left(1-X_{1}\right) V_{e}^{-1-\epsilon / 2}\right] .
\end{aligned}
$$

When we consider

$$
E_{\mu \nu}=\frac{1}{i \pi^{2} \Gamma(3)} \int d^{d} q \frac{q_{\mu} q_{\nu}}{\left(q^{2}+m_{1}^{2}\right) \cdots\left(\left(q+p_{1}+\cdots+p_{4}\right)^{2}+m_{5}^{2}\right)},
$$

the contribution proportional to $E_{0}(d=8)$ is

$$
E_{\mu \nu}=-\frac{E_{0}(d=8)}{4 B_{5}}\left[\delta_{\mu \nu}+\sum_{i, j=1}^{4} H_{i j}^{-1} p_{i \mu} p_{j \nu}\right]+\text { form factors } \quad N<5
$$


Since the four-vectors $p_{i \mu}$ span $d=4$ space-time the term proportional to $E_{0}(d=8)$ disappears and, therefore, there is no need to compute it. Indeed let us contract with $p_{l}, l=1, \ldots, 4$ to obtain

$$
p_{l \nu}\left(\delta_{\mu \nu}+\sum_{i, j=1}^{4} H_{i j}^{-1} p_{i \mu} p_{j \nu}\right)=p_{l \mu}-\sum_{i, j=1}^{4} H_{i j}^{-1} p_{i \mu} H_{i l}=0 .
$$

When we go to three powers of $q$ in the numerator the following result is valid:

$$
E_{\mu \nu \alpha}=-\frac{1}{2 B_{5}} \int d S_{4} V^{-1-\epsilon / 2}\left[\sum_{i} x_{i}\left\{\delta p_{i}\right\}_{\mu \nu \alpha}+\sum_{i \leq j \leq l} e_{i j l}^{3}\left\{p_{i} p_{j} p_{l}\right\}_{\mu \nu \alpha}\right]+\text { form factors } N<5
$$

where the coefficients $e^{3}$ are

$$
e_{i j l}^{3}=H_{i j}^{-1} x_{l} \quad+\quad \text { cyclic }
$$

and where we introduced

$$
\{\delta p\}_{\mu \nu \alpha}=\delta_{\mu \nu} p_{\alpha}+\delta_{\mu \alpha} p_{\nu}+\delta_{\nu \alpha} p_{\mu}, \quad\{p q k\}_{\mu \nu \alpha}=p_{\mu} q_{\nu} k_{\alpha}+\quad \text { cyclic },
$$

showing that $E_{0}(d=8)$ disappears again. It is straightforward to extend the demonstration up to five powers of loop momentum in the numerator. This is the maximum of non-contracted powers that we can have in a renormalizable theory and starting from six powers we will have numerators of the following structure,

$$
\left\{q \cdot p_{i}, q^{2}\right\} q_{\mu_{1}} \cdots q_{\mu_{5}}
$$

where scalar products can be simplified according to $q^{2}=[1]-m_{1}^{2}$ etc. There is only one case where the argument fails: suppose that one external line of momentum $p_{i}$ splits into two lines of momenta $p_{i j}, j=1,2$. Then scalar products $q \cdot p_{i j}$ do not occur in any of the propagators and in the final answer we would end up with evanescent operators like

$$
\frac{1}{\epsilon} \delta_{[-\epsilon]}^{\mu \nu} .
$$

We now describe the solution around $B_{5}=0$.

\subsection{Evaluation of $E_{0}$ when $B_{5} \approx 0$ and $E_{0}$ is regular}

If $B_{5} \approx 0$ and the condition $0<X_{i}<X_{i+1}<1, i=1, \cdots, 4$ is not satisfied $E_{0}$ is regular but the decomposition into a sum of boxes fails. We write

$$
E_{0}=\int d S_{4}(X)\left(x^{t} H x+B_{5}\right)^{-3-\epsilon / 2} .
$$

Since $E_{0}$ is regular at $B_{5}=0$ we perform a Taylor expansion in $B_{5}$ with coefficients $\mathcal{E}(n)$,

$$
\begin{gathered}
E_{0}=\frac{1}{2} \sum_{n=0}^{\infty}(n+1)(n+2) \mathcal{E}(n+3)\left(-B_{5}\right)^{n}, \\
\mathcal{E}(n)=\int d S_{4}(X)\left(x^{t} H x\right)^{-n-\epsilon / 2},
\end{gathered}
$$

with $n \geq 3$. Using

$$
\int d S_{4}(X)\left[1+\frac{x \partial_{x}}{2 n+\epsilon}\right]\left(x^{t} H x\right)^{-n-\epsilon / 2}=0,
$$


we easily obtain

$$
\mathcal{E}(n)=\frac{1}{4-2 n} \sum_{i=0}^{4} \int d S_{3}\left(X_{i}-X_{i+1}\right) Q^{-n-\epsilon / 2}(T \widehat{i i+1}),
$$

where, as usual, we introduced shifted arguments. If we denote $x^{t} H x$ with $Q\left(x_{1}, x_{2}, x_{3}, x_{4}\right)$ the secondary quadrics and the corresponding coefficients are

$$
\begin{aligned}
& Q(T \widehat{01}) \equiv Q_{1}=Q\left(1-X_{1}, x_{1}-X_{2}, x_{2}-X_{3}, x_{3}-X_{4}\right), \\
& Q(T \widehat{12}) \equiv Q_{2}=Q\left(x_{1}-X_{1}, x_{1}-X_{2}, x_{2}-X_{3}, x_{3}-X_{4}\right), \\
& Q(T \widehat{23}) \equiv Q_{3}=Q\left(x_{1}-X_{1}, x_{2}-X_{2}, x_{2}-X_{3}, x_{3}-X_{4}\right), \\
& Q(T \widehat{34}) \equiv Q_{4}=Q\left(x_{1}-X_{1}, x_{2}-X_{2}, x_{3}-X_{3}, x_{3}-X_{4}\right), \\
& Q(T \widehat{45}) \equiv Q_{5}=Q\left(x_{1}-X_{1}, x_{2}-X_{2}, x_{3}-X_{3},-X_{4}\right),
\end{aligned}
$$

and $X_{0}=1, X_{5}=0$. Again, each coefficient in the Taylor expansion is written as a combination of threefold integrals which can be evaluated with standard BT techniques. This will introduce sub-leading quadrics, i.e.

$$
Q_{i 1}=Q_{i}(\widehat{01})=Q_{i}\left(1, x_{1}, x_{2}\right),
$$

etc, and sub-subleading quadrics, i.e.

$$
Q_{i j 1}=Q_{i j}(\widehat{01})=Q_{i j}\left(1, x_{1}\right),
$$

etc, and also constant terms, e.g. $Q_{i j 1}(1,1)$ etc. At each step non-leading BT factors are introduced and the procedure fails when one of the sub-leading BT factors is zero. In this case, since $E_{0}$ is the sum of 5 terms of the form

$$
E_{0}^{i}=-\frac{1}{4} \sum_{n=0}^{\infty}(n+2) \int d S_{2} q_{i} Q_{i}^{-n-3}\left(-B_{5}\right)^{n},
$$

with $q_{i}$ constant, we rewrite the sum as

$$
\frac{1}{4} \sum_{n=0}^{\infty}(n+2) Q_{i}^{-n-3}\left(-B_{5}\right)^{n}=\frac{1}{2} \int_{0}^{1} d z\left(Q_{i}+B_{5} z\right)^{-3} .
$$

Likewise, by changing variables we obtain

$$
\int_{0}^{1} d z \int d S_{3} q_{i}\left(Q_{i}+B_{5} z\right)^{-3}=\int d S_{3}(X) \int_{0}^{1} d z q_{i}\left(x^{t} H_{i} x+B_{4 i}+B_{5} z\right)^{-3},
$$

where $B_{4 i}$ is the relevant sub-leading BT factor and we can apply a Mellin-Barnes splitting, followed by a sector decomposition, to $x^{t} H_{i} x$ and $B_{4 i}+B_{5} z^{2}$. For the Mellin-Barnes anti-transform the leading contribution comes from the pole at $s=3 / 2$ giving

$$
\int_{0}^{1} d z\left(B_{4 i}+B_{5} z\right)^{-3 / 2}=-\frac{2}{B_{5}}\left[\left(B_{4 i}+B_{5}\right)^{-1 / 2}-B_{4 i}^{-1 / 2}\right] .
$$

Alternatively we define $V_{0}\left(x_{1}, \ldots, x_{4}\right)=V\left(x_{1}, \ldots, x_{4}\right)-B_{5}$ and write down the BT relations corresponding to $B_{5} \neq 0$ and $B_{5}=0$ :

$$
\begin{gathered}
{\left[1+\frac{1}{4+\epsilon}(x-X) \partial_{x}\right]\left[V_{0}\left(x_{1}, \ldots, x_{4}\right)+B_{5}\right]^{-2-\epsilon / 2}=B_{5}\left[V_{0}\left(x_{1}, \ldots, x_{4}\right)+B_{5}\right]^{-3-\epsilon / 2},} \\
{\left[1+\frac{1}{4+\epsilon}(x-X) \partial_{x}\right] V_{0}^{-2-\epsilon / 2}\left(x_{1}, \ldots, x_{4}\right)=0 .}
\end{gathered}
$$


Then, after having summed each side of the two equations, we integrate by parts and set $\epsilon=0$, obtaining:

$$
E_{0}=\left.\frac{1}{4} \int d S_{2} \sum_{i=0}^{4}\left(X_{i}-X_{i+1}\right) B_{5}^{-1} V^{-2}(\widehat{i i+1})\right|_{\mathrm{sub}} .
$$

Hence the study of the scalar pentagon reduces again to the study of four-point functions. The tensor pentagons too can be treated analogously to the case $B_{5} \neq 0$.

\subsection{Evaluation of $E_{0}$ when $B_{5} \approx 0$ and $E_{0}$ is singular}

For the pentagon we are in a special situation even if $B_{5} \approx 0$ and the condition $X_{i}<X_{i-1}, i=1, \ldots, 5$ (with $X_{0}=1$ and $X_{5}=0$ ) is satisfied. Indeed, in this case, the BT algorithm already gives the correct leading behavior, $E_{0} \sim 1 / B_{5}$. This is easily seen by the Mellin-Barnes technique combined with a sector decomposition that would require to anti-transform the product

$$
\frac{\Gamma(s) \Gamma(3-s)}{s-2} \rho_{5}^{3-s}, \quad \rho_{5}=\frac{1}{B_{5}} .
$$

Closing the $s$ contour over the right-hand complex half-plane at infinity, thus selecting the poles at $s=2$ and $s=3+k, k \geq 0$, gives $\rho_{5}$ as the leading term. Therefore, in this case, we simply go on in treating the four-point functions according to their (sub-leading) BT factors.

\section{$9 \quad$ Infrared divergent $E_{0}$}

For the $E$-family, as long as $B_{5} \neq 0$, we can use Eq.(268) even for IR divergent configurations, therefore reducing the problem to the one of boxes. If instead $B_{5} \approx 0$ another procedure is more convenient. The physical relevance of the $E$-family in photonic virtual corrections to four-fermion production in $e^{+} e^{-}$ annihilation, as well as examples of the corresponding $E_{0}$ reduction, have been illustrated in 26].

Let us give an example for $E_{0}$ where we consider the configuration with

$$
p_{1}^{2}=p_{5}^{2}=-m^{2}, \quad m_{1}=0, \quad m_{2}=m_{5}=m, \quad m_{3}=m_{4}=M .
$$

If we introduce the notation,

$$
\begin{gathered}
{[1]=q^{2}, \quad[2]=\left(q+p_{1}\right)^{2}+m^{2}, \quad[3]=\left(q+p_{1}+p_{2}\right)^{2}+M^{2},} \\
{[4]=\left(q+p_{1}+p_{2}+p_{3}\right)^{2}+M^{2}, \quad[5]=\left(q-p_{5}\right)^{2}+m^{2},}
\end{gathered}
$$

then the following decomposition holds

$$
E_{0}^{\mathrm{rem}}=-\frac{1}{2}\left[\left(p_{1}+p_{2}\right)^{2}+M^{2}\right] E_{0}^{I R}+\frac{1}{2}\left(D_{0}^{15}+D_{0}^{23}\right),
$$

where the IR finite reminder is

$$
E_{0}^{\mathrm{rem}}=\frac{1}{i \pi^{2}} \int d^{d} q \frac{q \cdot\left(q+p_{1}+p_{2}\right)}{[1] \cdots[5]}
$$

and where the two $D_{0}$ functions are specified by their list of arguments:

$$
\begin{aligned}
& D_{0}^{15} \equiv D_{0}\left(p_{2}^{2}, p_{3}^{2}, p_{4}^{2},\left(p_{1}+p_{5}\right)^{2},\left(p_{2}+p_{3}\right)^{2},\left(p_{3}+p_{4}\right)^{2}, m, M, M, m\right), \\
& D_{0}^{23} \equiv D_{0}\left(-m^{2},\left(p_{2}+p_{3}\right)^{2}, p_{4}^{2},-m^{2},\left(p_{4}+p_{5}\right)^{2},\left(p_{1}+p_{5}\right)^{2}, 0, m, M, m\right) .
\end{aligned}
$$


As done in Section 7 the reminder is computed without simplifications between numerator and denominator. In particular, if $B_{5} \neq 0$ we use the method of Section 8.1; otherwise, for $B_{5}=0$ and $E_{0}^{\text {rem }}$ regular (singular) we use the results of Section 8.3 (Section 8.4). The classification of arbitrary IR divergent $E_{0}$ functions follows from IR power counting similarly to the discussion of Section 7.1. In all cases the IR divergent part is expressed through $C_{0}$ functions that are contained in some of the $D_{0}$ which appear in decompositions of the type expressed by Eq.(313).

\section{Six-point functions (F-family)}

To compute the generic, scalar, six-point function [27] we introduce more compact notations:

$$
H_{N, i j}^{-1}=\frac{\Delta_{N, i j}}{G_{N}},
$$

where $H_{N}$ is the $(N-1) \times(N-1)$ matrix with elements $-p_{i} \cdot p_{j}, G_{N}=\operatorname{det} H_{N}$ is its determinant and $\Delta_{N, i j}$ is the co-determinant of the element $H_{i j}$. The quadric $V_{N}$ will be written as $V_{N}=x^{t} H_{N} x+2 K_{N}^{t} x+L_{N}$. It follows that

$$
B_{N}=\frac{b_{N}}{G_{N}}, \quad b_{N}=G_{N} L_{N}-K_{N}^{t} \Delta_{N} K_{N}, \quad X_{N}=\frac{\mathcal{X}_{N}}{G_{N}}, \quad \mathcal{X}_{N}=-\Delta_{N} K_{N} .
$$

Furthermore, $L_{N}=m_{1}^{2}$ and $K_{N}$ has elements

$$
K_{N i}=\frac{1}{2}([i+1]-[i]), \quad i=1, \ldots, N-1,
$$

where [1] $=m_{1}^{2}$ and $[j]=\left(p_{1}+\cdots+p_{j-1}\right)^{2}+m_{j}^{2}$, with $j=2, \ldots, N$. Since the scalar hexagon is UV finite we can work in $d=4$ dimensions where $G_{6}=0$. Therefore, one BT iteration gives

$$
b_{6} F_{0}=\frac{1}{6} \int d S_{4} \sum_{i=0}^{5}\left(\mathcal{X}_{i}-\mathcal{X}_{i+1}\right) V_{6 i}^{-3-\epsilon / 2},
$$

where $\mathcal{X}_{0}=\mathcal{X}_{6}=0$ and where

$$
\begin{aligned}
V_{60} & =V(\widehat{01})=V\left(1, x_{1}, \cdots, x_{4}\right), \\
V_{61} & =V(\widehat{12})=V\left(x_{1}, x_{1}, \cdots, x_{4}\right), \\
V_{62} & =V(\widehat{23})=V\left(x_{1}, x_{2}, x_{2}, \cdots, x_{4}\right), \\
& \cdots \\
V_{65} & =V(\widehat{56})=V\left(x_{1}, \cdots, x_{4}, 0\right),
\end{aligned}
$$

and the scalar hexagon is the sum of six pentagons, i.e. of 30 boxes [24].

In computing the hexagon we have to decide about the input parameter set (hereafter IPS). Clearly internal masses, $m_{1}, \cdots, m_{6}$ belong to the IPS and for the rest we prefer to use invariants. For a total of $N$ momenta we have $3 N-10$ independent invariants which is considerably less than the number of inner products. The energy-momentum conservation leads to a number of linear relations among the inner products, of which a typical example is that connecting $s, t, u$ for the box. Secondly, the dimensionality constraints lead to quadratic relations. For the hexagon there is just one, expressed by $G_{6}=0$. The complete set of invariants will be (remember that in our conventions all momenta are flowing inwards) $s_{i j k \cdots}=-\left(p_{i}+p_{j}+p_{k}+\cdots\right)^{2}$, and we will select 8 of them for our IPS. 


\subsection{Form factors in the F-family}

Consider the integral

$$
F_{\mu}=\frac{1}{i \pi^{2} \Gamma(4)} \int d^{d} q \frac{q_{\mu}}{\left(q^{2}+m_{1}^{2}\right) \cdots\left(\left(q+p_{1}+\cdots+p_{5}\right)^{2}+m_{6}^{2}\right)}=\sum_{i=1}^{5} F_{1 i} p_{i \mu} .
$$

We immediately obtain

$$
F_{1 i}=\frac{\mathcal{X}_{i}}{6 b_{6}} \int d S_{5} V_{6}^{-3-\epsilon / 2}(\{x\})+F_{1 i}^{4}
$$

where $F_{1 i}^{4}$ is a fourfold integral. Therefore there is a piece in $F_{\mu}$ proportional to $F_{0}(d=6)$, where the coefficient is

$$
\sum_{i=1}^{5} \mathcal{X}_{i} p_{i \mu}
$$

In order to prove that this coefficient is zero it is enough to contract it with a set of vectors $p_{l}$ that span $d=4$ space-time. In this way we obtain

$$
-\sum_{i=1}^{5} \Delta_{i j} K_{j} p_{i} \cdot p_{l}=\sum_{i=1}^{5} \Delta_{i j} K_{j} H_{i l} .
$$

Since $\Delta_{i j}$ is the co-determinant of the element $H_{i j}$ we can use Laplace's theorem, $\Delta_{i j} H_{i l}=\delta_{j l} G$, to obtain

$$
\sum_{i=1}^{5} \Delta_{i j} K_{j} H_{i l}=G K_{l}=0
$$

due to the vanishing of the Gram's determinant. For two or more powers of loop momenta in the numerator we need a new relation; let $d=4-\epsilon$ and consider

$$
\left(x-\frac{\mathcal{X}}{G}\right) V^{\mu-1}=\frac{1}{2 \mu} \frac{\Delta}{G} \partial V^{\mu}, \quad G=\sum_{n=1}^{\infty} G^{(n)} \epsilon^{n},
$$

where $\mu$ is arbitrary. We obtain

$$
\mathcal{X} V^{\mu-1}=-\frac{1}{2 \mu} \Delta \partial V^{\mu}, \quad\left(G^{(1)}\right)^{2} x V^{\mu-1}+G^{(2)}\left(\mathcal{X} V^{\mu-1}+\frac{1}{2 \mu} \Delta \partial V^{\mu}\right)=0,
$$

etc. With the help of Eq.(327) we can show that the part proportional to $F_{0}(d=8)$ in $F_{\mu \nu}$ has a coefficient

$$
\sum_{i, j=1}^{5} \Delta_{i j} p_{i \mu} p_{j \nu}
$$

which contracted with $p_{l}, l=1, \cdots, 5$ gives

$$
\sum_{i, j=1}^{5} \Delta_{i j} p_{i \mu} p_{j} \cdot p_{l}=-\sum_{i, j=1}^{5} \Delta_{i j} H_{j l} p_{i \mu}=-G p_{l \mu}=0 .
$$


In computing form factors of the $F$-family we will use results already obtained for the $E$-family showing that, once more, everything is reducible to form factors of the $D$-family. For instance for $F_{\mu}$ we use

$$
\sum_{i=1}^{5} \mathcal{X}_{i j} p_{j \mu}=0, \quad \frac{X_{i}}{B_{6}}=\frac{\mathcal{X}_{i}}{b_{6}}, \quad G_{6}=0,
$$

and also

$$
V^{-4-\epsilon / 2}=\frac{1}{B_{6}}\left[1+\frac{(x-X) \partial}{6+\epsilon}\right] V^{-3-\epsilon / 2}, \quad \text { or } \quad V^{-4-\epsilon / 2}=-\frac{1}{b_{6}} \frac{\mathcal{X} \partial}{6+\epsilon} V^{-3-\epsilon / 2} .
$$

Furthermore, secondary quadrics are

$$
\begin{aligned}
& V(\widehat{01})=V\left(1, x_{1}, x_{2}, x_{3}, x_{4}\right)=V_{1}\left(x_{1}, x_{2}, x_{3}, x_{4}\right), \\
& V(\widehat{12})=V\left(x_{1}, x_{1}, x_{2}, x_{3}, x_{4}\right)=V_{2}\left(x_{1}, x_{2}, x_{3}, x_{4}\right), \\
& V(\widehat{23})=V\left(x_{1}, x_{2}, x_{2}, x_{3}, x_{4}\right)=V_{3}\left(x_{1}, x_{2}, x_{3}, x_{4}\right), \\
& V(\widehat{34})=V\left(x_{1}, x_{2}, x_{3}, x_{3}, x_{4}\right)=V_{4}\left(x_{1}, x_{2}, x_{3}, x_{4}\right), \\
& V(\widehat{45})=V\left(x_{1}, x_{2}, x_{3}, x_{4}, x_{4}\right)=V_{5}\left(x_{1}, x_{2}, x_{3}, x_{4}\right), \\
& V(\widehat{56})=V\left(x_{1}, x_{2}, x_{3}, x_{4}, 0\right)=V_{6}\left(x_{1}, x_{2}, x_{3}, x_{4}\right),
\end{aligned}
$$

to which we apply a standard BT algorithm

$$
V_{i}^{-3-\epsilon / 2}=\frac{1}{B_{5 i}}\left[1+\frac{1}{4+\epsilon}\left(x_{j}-X_{i j}\right) \partial_{j}\right] V_{i}^{-2-\epsilon / 2}, \quad i=1, \cdots, 6,
$$

or a special BT algorithm,

$$
x_{j} V_{i}^{-2-\epsilon / 2}=X_{i j} V_{i}^{-2-\epsilon / 2}-\frac{1}{2+\epsilon}\left(H_{i}\right)_{j l}^{-1} \partial_{l} V_{i}^{-1-\epsilon / 2},
$$

to show that $F_{\mu}$ is given by a combination of 813 threefold integrals, i.e. form factors of the $D$-family.

\subsection{Special configurations for $F_{0}$}

If we have $b_{6}=-K^{t} \Delta K=0$ on top of $G_{6}=0$ then Eq. (319) cannot be applied. In this case we can proceed as follows: since the vectors $p_{i}$ are linearly dependent, we substitute

$$
p_{5 \mu}=-\sum_{i=1}^{4} \frac{\mathcal{X}_{i}}{\mathcal{X}_{5}} p_{i \mu}
$$

into the expression of $V$ and show that

$$
\sum_{i, j=1}^{5} H_{i j} x_{i} x_{j}=\sum_{i, j=1}^{4} H_{i j}^{(4)} X_{i} X_{j}, \quad X_{i}=x_{i}-\frac{\mathcal{X}_{i}}{\mathcal{X}_{5}} x_{5},
$$

where $H^{(4)}$ is a $4 \times 4$ matrix with elements $-p_{i} \cdot p_{j}$. Likewise we have

$$
\sum_{i=1}^{5} K_{i} x_{i}=\sum_{i=1}^{4} K_{i} X_{i}+\frac{b_{6}}{\mathcal{X}_{5}} x_{5}
$$


If we define

$$
L_{i}=\left(1-r_{i}\right) x_{5}=\left(1-\frac{\mathcal{X}_{i}}{\mathcal{X}_{5}}\right) x_{5}, \quad U_{i}=x_{i-1}+\left(r_{i-1}-r_{i}\right) x_{5},
$$

with $x_{0}=1$ and $r_{0}=0$, the expression for $F_{0}$ can be cast into the following form:

$$
F_{0}=\int_{0}^{1} d x_{5} \mathcal{F}_{0}\left(x_{5}\right), \quad \mathcal{F}_{0}\left(x_{5}\right)=\prod_{i=1}^{4} \int_{L_{i}}^{U_{i}} d x_{i}\left(Q_{4}+2 \frac{b_{6}}{\mathcal{X}_{5}} x_{5}\right)^{-4-\epsilon / 2},
$$

where $Q_{4}$ is a quadric in four variables, i.e.

$$
Q_{4}=\sum_{i, j=1}^{4} H_{i j}^{(4)} x_{i} x_{j}+2 \sum_{i=1}^{4} K_{i} x_{i}+L
$$

To this quadric is associated a BT factor $B_{4}$ so that we can apply the BT algorithm to Eq.(339) with a total BT factor $B_{4}+2 b_{6} / \mathcal{X}_{5} x_{5}$.

\section{Conclusions}

In this paper we presented a detailed investigation of the algorithms, based on the Bernstein-Tkachov theorem [1], which form the basis for a fast and reliable numerical integration of one-loop multi-leg (up to six in this paper) diagrams. The rationale for this work is represented by the need of encompassing a number of problems that one encounters in assembling a calculation of some complicated process, e.g. full one-loop corrections to $e^{+} e^{-} \rightarrow 4$ fermions. Furthermore, in any attempt to compute physical observables at the two-loop level, we will have to include the one-loop part, and it is rather obvious that the two pieces should be treated on equal footing.

Finally, our method represents a new strategy for the so-called problem of reduction of tensor integrals (for additional alternatives we refer to [24],25] and [28]).

All algorithms that aim to compute Feynman diagrams numerically are based on some manipulation of the original integrands that brings the final answer into something smooth. This has the consequence of bringing the original (Landau) singularity of the diagram into some overall denominator and, usually, the method overestimates the singular behavior around some threshold. In these regions an alternative derivation is needed. Instead of using the method of asymptotic expansions [29], we introduced a novel algorithm based on a Mellin-Barnes decomposition of the singular integrand, followed by a sector decomposition that allows us to write the Laurent expansion around threshold.

Particular care has been devoted to analyze those situations where a sub-leading singularity may occur, and to properly account for those cases where the algorithm cannot be applied because the corresponding BT factor is zero although the singular point in parametric space does not belong to the integration domain.

Clearly, no numerical evaluation of Feynman diagrams should be attempted unless we have a detailed knowledge of their analytic structure; in other words, we must know beforehand where the real singularities are sitting, which apparent singularity can be the origin of numerical instabilities, and what to do when one of these cases is met.

One of the main by-products of the BT approach, Eq.(1), is that any diagram $G$ has an associated factor $B_{G}$ which is immediately computed for any one-loop diagram and gives information about the leading solution of the corresponding set of Landau equations (these solutions are notoriously hard to derive with standard methods [30]). Technically speaking, $B_{G}=0$ guarantees that the Landau equations 
admit a proper solution. Repeated applications of the algorithm, as explained for instance in Section 6.1, will also introduce sub-leading $B_{G \mid S L}$-factors whose zeros correspond to sub-leading Landau singularities. Likewise, our solution around $B_{G}=0$ will introduce sub-leading $B$-coefficients of a second kind (as explained, for instance, in the discussion after Eq.(84)), not associated with Landau singularities of the reduced diagrams.

When these pieces of knowledge are at hand, we can automatize our calculations, even without having to worry in advance about the physical nature [31] of the singular points (a much more difficult assignment): it will be enough to be able to handle all cases and let the program decide when $B_{G}=0$ corresponds to a singular behavior or when a coincidence occurs. Furthermore, we have a conjecture stating that when $B_{G}=0$, then the simultaneous occurrence of $B_{G \mid S L}=0$ is not physical, but we have not been able to prove it. Instead we decided to cover also this case, so that our expressions for the one-loop diagrams are quite general and not limited to the physical region of a specific process.

In general it is known that when a given Landau singularity curve touches one of its sub-leading singularity curves the determination of the nature of the singularity breaks down completely (because the $\alpha_{i}$ 's are no longer uniquely determined) and changes suddenly and drastically. Similarly, our analysis via the multiple Mellin - Barnes techniques (cf. Eqs.(92)-(93) and Eq.(221)), dealing with the coincidence

$B_{G}=B_{G \mid S L}=0$ (where $B_{G \mid S L}$ is of second kind), represents a novel result. Alternatively, we have been able to derive new integral representations that bypass the use of Laurent expansions.

Finally we have given a description of infrared divergent one-loop virtual configurations in the framework of dimensional regularization: here both the residue of the infrared pole and the infrared finite remainder are cast into a form that can be safely computed numerically.

The collection of formulas that cover all corners of phase space have been translated into a set of FORM codes [32] and the output has been used to create a FORTRAN code whose technical description will be given elsewhere.

\section{Acknowledgments}

For the idea of presenting a comprehensive discussion of all algorithms needed in the numerical evaluation of one-loop diagrams we gratefully acknowledge the incitation of Ansgar Denner, Stefan Dittmaier and Stanislaw Jadach. G. P. would like to express his gratitude to Fyodor Tkachov for important discussions on the general idea of evaluating multi-loop Feynman diagrams numerically, and to recognize the critical spur of Dima Bardin. M. P. would like to thank J. Gasser for enlightening discussions on the analytic properties of Feynman diagrams. 


\section{A A master integral}

In this appendix we calculate the master integral

$$
I_{(\alpha, \beta)}(a, b)=\int_{0}^{1} d x x^{\beta}\left(a x^{2}+b-i \delta\right)^{-\alpha},
$$

where $a$ and $b$ are real numbers with $b$ approaching 0 and $a$ far from 0 . If $\beta>2 \alpha-1$ the integral is not divergent, otherwise we split the integral into two pieces: the first one contains the divergent part as $b \rightarrow 0$, while the second is finite:

$$
I_{(\alpha, \beta)}(a, b)=\int_{0}^{\infty} d x x^{\beta}\left(a x^{2}+b-i \delta\right)^{-\alpha}-\int_{1}^{\infty} d x x^{\beta}\left(a x^{2}+b-i \delta\right)^{-\alpha} .
$$

Since we are in the limit of $\delta \rightarrow 0$, we may write $a x^{2}+b-i \delta=(a-i \delta) x^{2}+b-i \delta$. Next we perform the following transformations in the two integrals respectively: $x \rightarrow x^{1 / 2}$ and $x \rightarrow x^{-1}$ and obtain

$$
I_{(\alpha, \beta)}(a, b)=\frac{1}{2} \int_{0}^{\infty} d x x^{\frac{\beta-1}{2}}[(a-i \delta) x+b-i \delta]^{-\alpha}-\int_{0}^{1} d x x^{2 \alpha-\beta-2}\left[a-i \delta+(b-i \delta) x^{2}\right]^{-\alpha} .
$$

In the first integral an additional change of variable is performed:

$$
x \rightarrow \frac{b-i \delta}{a-i \delta} \frac{1-x}{x}
$$

and we make use of the following property:

$$
\left(\frac{b-i \delta}{a-i \delta}\right)^{\mu}=\frac{(b-i \delta)^{\mu}}{(a-i \delta)^{\mu}} .
$$

Hence the final result is:

$$
I_{(\alpha, \beta)}(a, b)=\frac{(b-i \delta)^{-\alpha+\frac{\beta+1}{2}}}{2(a-i \delta)^{\frac{\beta+1}{2}}} B\left(\frac{\beta+1}{2}, \alpha-\frac{\beta+1}{2}\right)-\int_{0}^{1} d x x^{2 \alpha-\beta-2}\left[a-i \delta+(b-i \delta) x^{2}\right]^{-\alpha} .
$$

The second term is well defined only for $\beta<2 \alpha-1$ and we perform an analytic continuation to the region $\beta<2 \alpha+1$ (with $a, b \rightarrow a, b-i \delta$ ):

$$
I_{(\alpha, \beta)}(a, b)=\frac{b^{-\alpha+\frac{\beta+1}{2}}}{2 a^{\frac{\beta+1}{2}}} B\left(\frac{\beta+1}{2}, \alpha-\frac{\beta+1}{2}\right)-\frac{a^{-\alpha}}{2 \alpha-\beta-1}-\int_{0}^{1} d x x^{2 \alpha-\beta}\left\{\frac{\left[a+b x^{2}\right]^{-\alpha}}{x^{2}}\right\}_{++}
$$

From Eq. 347) we see that the two divergent terms cancel out also when $\beta=2 \alpha-1$. For $\alpha=n$ and $\beta=2 n-1$ we get

$$
I_{(\alpha, \beta)}(a, b)=-\frac{a^{-n}}{2}\left(\ln \frac{b}{a}+\sum_{j=1}^{n-1} \frac{1}{j}\right)-\int_{0}^{1} d x\left\{\frac{\left[a+b x^{2}\right]^{-n}}{x}\right\}_{+} .
$$



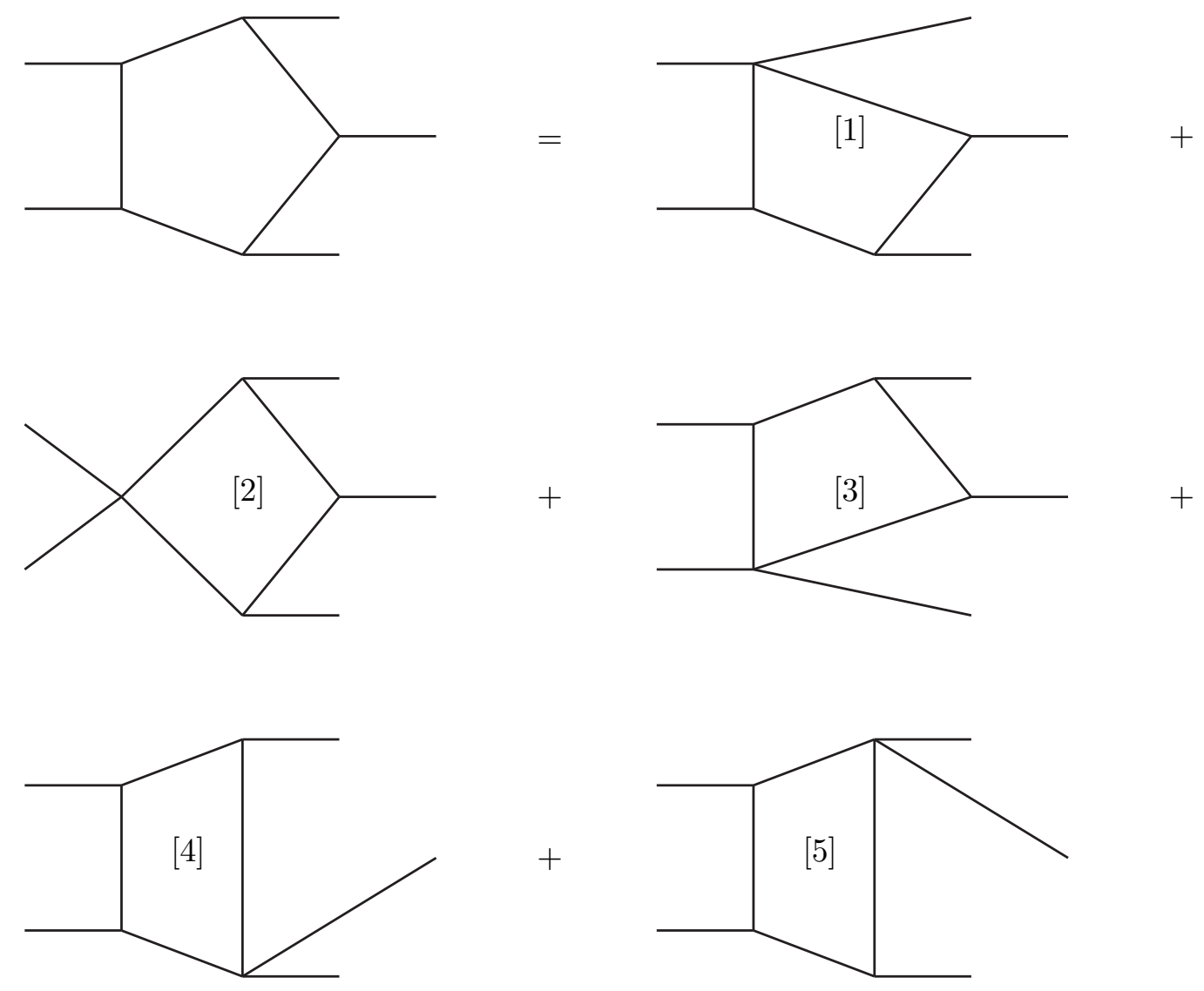

Figure 5: Diagrammatical representation of the BT algorithm of Eq.(268) for the pentagon. The symbol $[i]$ denotes multiplication of the corresponding box by a factor $w_{i} /\left(4 B_{5}\right)$. 


\section{References}

[1] G. 't Hooft and M. Veltman, Nucl. Phys. B 153 (1979) 365.

[2] A.V. Kotikov, Phys. Lett. B 254 (1991) 158;

A.V. Kotikov, Phys. Lett. B 267 (1991) 123.

[3] L.D. Landau, Nucl. Phys. 13 (1959) 181.

[4] R.J. Eden, P.V. Landshoff, D.I. Olive, and J.C. Polkinghorne, The Analytic S-Matrix, Cambridge Univ. Press, 1966.

[5] W.L. van Neerven and J.A. Vermaseren, Phys. Lett. B 137 (1984) 241;

Z. Bern, L.J. Dixon and D.A. Kosower, Nucl. Phys. B 412 (1994) 751 arXiv:hep-ph/9306240;

A.I. Davydychev, J. Math. Phys. 33 (1992) 358.

[6] A. Vicini, talk given at Loopfest, Brookhaven National Laboratory, Upton, NY, May 9-10, 2002 (http://www.pas.rochester.edu/ dow/loopfest/parallel.html).

[7] W. Beenakker et al., Nucl. Phys. B 500 (1997) 255 arXiv:hep-ph/9612260.

[8] G. Passarino and M. Veltman, Nucl. Phys. B 160 (1979) 151;

D. Bardin and G. Passarino, The standard model in the making: Precision study of the electroweak interactions, Oxford, UK: Clarendon (1999).

[9] G. Passarino, Nucl. Phys. B 619 (2001) 257 arXiv:hep-ph/0108252;

G. Passarino and S. Uccirati, Nucl. Phys. B 629 (2002) 97 arXiv:hep-ph/0112004.

[10] D.Y. Bardin, L.V. Kalinovskaya and F.V. Tkachov, hep-ph/0012209,

G. Passarino, hep-ph/0101299.

[11] F.V. Tkachov, Nucl. Instrum. Meth. A 389 (1997) 309 hep-ph/9609429];

L.N. Bertstein, Functional Analysis and its Applications, 6(1972)66.

[12] A. Erdelyi et al., Higher Transcendental Functions, vol. 2, Bateman Manuscript Project, McGrawHill, 1953;

L.J. Slater, Generalized Hypergeometric Functions, Cambridge Univ. Press, 1966.

[13] T. Binoth and G. Heinrich, Nucl. Phys. B 585 (2000) 741 [arXiv:hep-ph/0004013;

M. Roth and A. Denner, Nucl. Phys. B 479 (1996) 495 arXiv:hep-ph/9605420;

K. Hepp, Commun. Math. Phys. 2 (1966) 301.

[14] J. Fujimoto, Y. Shimizu, K. Kato and Y. Oyanagi, Prog. Theor. Phys. 87 (1992) 1233;

J. Fujimoto, Y. Shimizu, K. Kato, N. Nakazawa and T. Kaneko, prepared for 10th International Workshop on High-energy Physics and Quantum Field Theory (NPI MSU 95), Zvenigorod, Russia, 19-26 Sep 1995;

F. Caravaglios, Nucl. Phys. B 589 (2000) 475 arXiv:hep-ph/0004030;

R. Easther, G. Guralnik and S. Hahn, Phys. Rev. D 61 (2000) 125001 arXiv:hep-ph/9903255;

M. Steinhauser, Phys. Rept. 364 (2002) 247 arXiv:hep-ph/0201075. 
[15] G. Montagna, F. Piccinini, O. Nicrosini, G. Passarino and R. Pittau, Comput. Phys. Commun. 76 (1993) 328;

G. Montagna, F. Piccinini, O. Nicrosini, G. Passarino and R. Pittau, Nucl. Phys. B 401 (1993) 3.

[16] E.E. Boos and A.I. Davydychev, Theor. Math. Phys. 89 (1991) 1052 [Teor. Mat. Fiz. 89 (1991) 56]; A.I. Davydychev and J.B. Tausk, Nucl. Phys. B 397 (1993) 123;

S. Bauberger, F.A. Berends, M. Bohm and M. Buza, Nucl. Phys. B 434 (1995) 383 arXiv:hepph/9409388;

A.I. Davydychev and J.B. Tausk, Phys. Rev. D 53 (1996) 7381 arXiv:hep-ph/9504431.

[17] R. Karplus, C. M. Sommerfield and E. H. Wichmann, Phys. Rev. 111 (1958) 1187;

G. Källen and A. S. Wightman, K. Dan. Vidensk. Selsk. Mat. - Fys. Skr. 1 N0. 6 (1958).

[18] R. E. Cutkosky, J. Math. Phys. 1 (1960) 429.

[19] G. Devaraj and R.G. Stuart, Nucl. Phys. B 519 (1998) 483 arXiv:hep-ph/9704308.

[20] L. Lewin, Poly-logarithms and Associated Functions, North Holland (New York 1981).

[21] W. Beenakker and A. Denner, Nucl. Phys. B 338 (1990) 349.

[22] H.D. Abarbanel and C. Itzykson, Phys. Rev. Lett. 23 (1969) 53;

M. Levy and J. Sucher, Phys. Rev. 186 (1969) 1656.

[23] G. Sterman, An Introduction to Quantum Field Theory, Cambridge Univ. Press, 1993.

[24] J. Fleischer, F. Jegerlehner and O.V. Tarasov, Nucl. Phys. B 566 (2000) 423 arXiv:hep-ph/9907327;

O. V. Tarasov, Acta Phys. Polon. B 29 (1998) 2655 arXiv:hep-ph/9812250;

Z. Bern, L.J. Dixon and D.A. Kosower, Nucl. Phys. B 412 (1994) 751 arXiv:hep-ph/9306240.

[25] T. Binoth, J.P. Guillet and G. Heinrich, Nucl. Phys. B 572 (2000) 361 arXiv:hep-ph/9911342;

Z. Bern, L. J. Dixon and D. A. Kosower, Phys. Rev. Lett. 70 (1993) 2677 [arXiv:hep-ph/9302280].

[26] A. Denner, S. Dittmaier and M. Roth, Nucl. Phys. B 519 (1998) 39 arXiv:hep-ph/9710521.

[27] T. Binoth, J.P. Guillet, G. Heinrich and C. Schubert, Nucl. Phys. B 615 (2001) 385 arXiv:hep$\mathrm{ph} / 0106243$.

[28] C. Bauer and H.S. Do, Comput. Phys. Commun. 144 (2002) 154 arXiv:hep-ph/0102231;

S. Weinzierl, Phys. Lett. B 450 (1999) 234 arXiv:hep-ph/9811365;

A.I. Davydychev, Phys. Lett. B 263 (1991) 107.

[29] F.V. Tkachov, in Quarks-82 Proc. Int. Seminar, Eds: A.N. Tavkhelidze et al. INR, the USSR Acad. Sci. Moscow, 1982; Euclidean asymptotic of Feynman integrals, preprint INR P-332 (Moscow 1984); Int. J. Mod. Phys. A8 (1993) 2047; Sov. J. Part. Nuclei 25 (1994) 649.

[30] A. C. Wu, Phys. Rev. D 9 (1974) 370.

[31] D. Kershaw, Phys. Rev. D 5 (1972) 1976.

[32] J.A. Vermaseren, math-ph/0010025. 